Florida International University FIU Digital Commons

FIU Electronic Theses and Dissertations

University Graduate School

6-22-2015

\title{
Factors Affecting Adult Mental Rotation Performance
}

Alina Nazareth

Florida International University, alina.nazareth@gmail.com

DOI: $10.25148 /$ etd.FIDC000115

Follow this and additional works at: https://digitalcommons.fiu.edu/etd

Part of the Developmental Psychology Commons

\section{Recommended Citation}

Nazareth, Alina, "Factors Affecting Adult Mental Rotation Performance" (2015). FIU Electronic Theses and Dissertations. 2185.

https://digitalcommons.fiu.edu/etd/2185

This work is brought to you for free and open access by the University Graduate School at FIU Digital Commons. It has been accepted for inclusion in FIU Electronic Theses and Dissertations by an authorized administrator of FIU Digital Commons. For more information, please contact dcc@fiu.edu. 


\section{FLORIDA INTERNATIONAL UNIVERSITY \\ Miami, Florida}

FACTORS AFFECTING ADULT MENTAL ROTATION PERFORMANCE

A dissertation submitted in partial fulfillment of

the requirements for the degree of

DOCTOR OF PHILOSOPHY

in

PSYCHOLOGY

by

Alina Nazareth 
To: Dean Michael R. Heithaus

College of Arts and Sciences

This dissertation, written by Alina Nazareth, and entitled Factors Affecting Adult Mental Rotation Performance, having been approved in respect to style and intellectual content, is referred to you for judgment.

We have read this dissertation and recommend that it be approved.

Anthony Dick

Asia Eaton

Angela Laird

Shannon M. Pruden, Major Professor

Date of Defense: June 22, 2015

The dissertation of Alina Nazareth is approved.

\begin{tabular}{r} 
Dean Michael R. Heithaus \\
College of Arts and Sciences \\
\hline Dean Lakshmi N. Reddi \\
University Graduate School
\end{tabular}

Florida International University, 2015 
C) Copyright 2015 by Alina Nazareth

All rights reserved. 


\section{DEDICATION}

I dedicate this dissertation to my family. The force of your love transcends the thousands of miles that separate us and gives me the courage to follow my dreams, wherever they may take me. 


\section{ACKNOWLEDGMENTS}

This dissertation could not have been possible without the ideas, support, feedback and encouragement of my mentor Dr. Shannon M. Pruden. Dr. Pruden has spent countless hours brainstorming research ideas, discussing experimental designs and analyses, and editing manuscripts to prepare me for a career in research and academia. I would like to extend my sincere gratitude to her for the time and effort invested in my professional development. I will always be grateful to her for introducing me to spatial research, a topic so dear to my heart. The passion and dedication she brings to her research has always inspired me to keep learning and improving. I would like to express my appreciation for my dissertation committee members Drs. Anthony Dick, Asia Eaton, and Angela Laird who have provided invaluable guidance and insight throughout the dissertation journey.

I would like to acknowledge the financial support of the Florida International University Dissertation Year Fellowship (DYF) that allowed me to dedicate time to my dissertation research during the final two semesters.

A very special thank you to my undergraduate research assistants Marcela Ramos, Nicole Matos, Ifrah Waheed, Anaelle Labi, Wellington Humes and my high-school intern Virginia Guerra, who have worked tirelessly to help me collect my dissertation data. Finally, I would like to thank my fellow graduate students Andrew Laginess, Erin Hedemann, Allison Mugno, Rosalie Odean, Carla Abad and Iris Broce for being my family away from home. 


\begin{abstract}
OF THE DISSERTATION
FACTORS AFFECTING ADULT MENTAL ROTATION PERFORMANCE
\end{abstract}

by

Alina Nazareth

Florida International University, 2015

Miami, Florida

Professor Shannon M. Pruden, Major Professor

Research on mental rotation has consistently found sex differences, with males outperforming females on mental rotation tasks like the Vandenberg and Kuse (1978) mental rotation test (MRT; D. Voyer, Voyer, \& Bryden, 1995). Mental rotation ability has been found to be enhanced with experience (Nazareth, Herrera \& Pruden, 2013) and training (Wright, Thompson, Ganis, Newcombe, \& Kosslyn, 2008) and the effects of training have been found to be transferable to other spatial tasks (Wright et al., 2008) and sustainable for months (Terlecki, Newcombe, \& Little, 2008). Although, we now are fairly certain about the malleability of spatial tasks and the role of spatial activity experience, we seem to have undervalued an important piece of the puzzle. What is the mechanism by which experiential factors enhance mental rotation performance? The current dissertation sought to address this gap in the literature by examining cognitive strategy selection as a possible mechanism by which experiential factors like early spatial activity experience enhance mental rotation performance. A total of 387 adult university students were randomly assigned to one of three experimental conditions. The three experimental conditions differed in the amount and type of non-spatial information present in the task stimuli. Participant eye movement was recorded using a Tobii X60 eye 
tracker. Study I investigated the different types of cognitive strategies selected during mental rotation, where eye movement patterns were used as indicators of the underlying cognitive strategies. A latent profile analysis revealed two distinct eye movement patterns significantly predicting mental rotation performance. Study II examined the role of early spatial activity experience in mental rotation performance. Male sex-typed spatial activities were found to significantly mediate the relation between participant sex and mental rotation performance. Finally, Study III examined the developmental role of early spatial activity experience in cognitive strategy selection and strategy flexibility to enhance mental rotation performance. Strategy flexibility was found to be significantly associated with mental rotation performance. Male sex-typed spatial activity experiences were found to be significantly associated with cognitive strategy selection but not strategy flexibility. Implications for spatial training and educational pedagogy in the STEM fields are discussed. 


\section{TABLE OF CONTENTS}

CHAPTER

PAGE

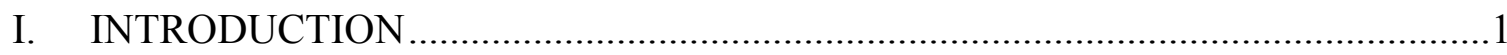

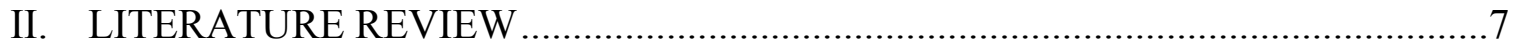

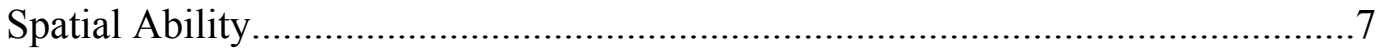

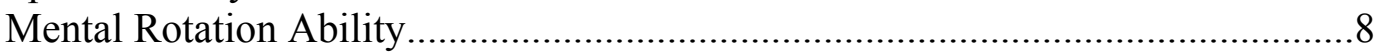

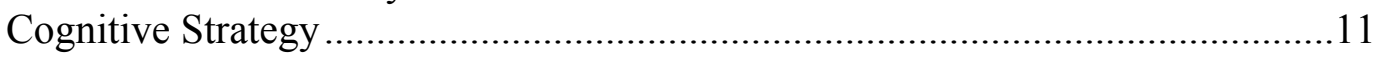

A Neuroscience Perspective ............................................................................13

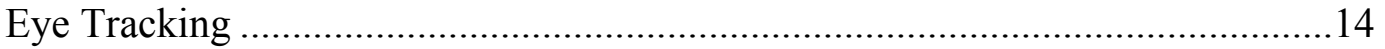

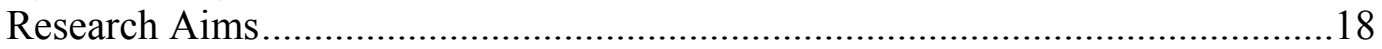

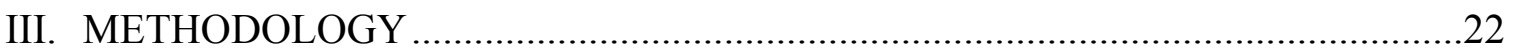

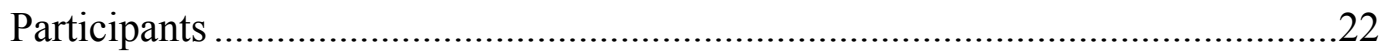

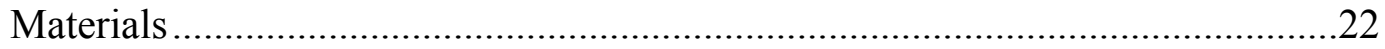

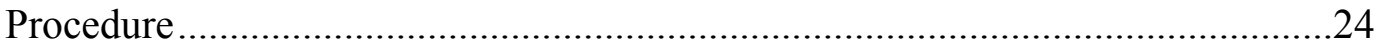

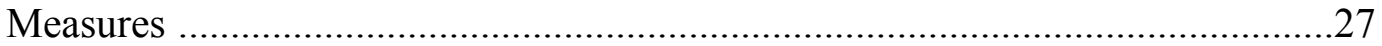

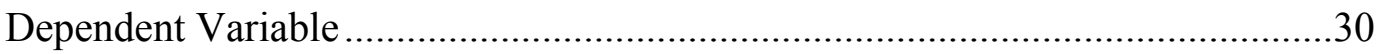

Independent and Mediating Variables...............................................................

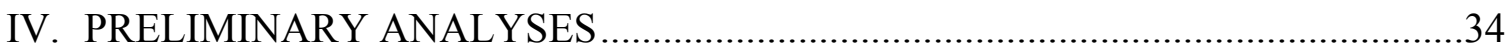

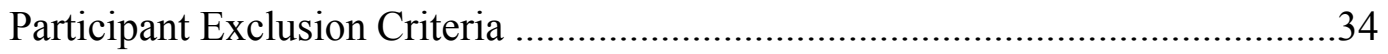

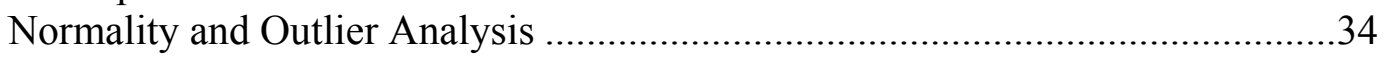

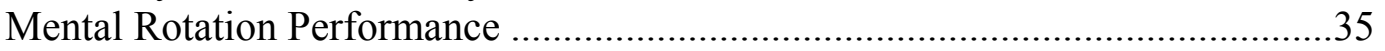

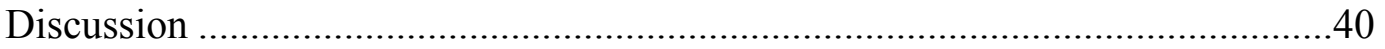

V. STUDY I COGNITIVE STRATEGY SELECTION IN MENTAL ROTATION ....42

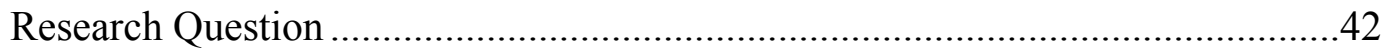

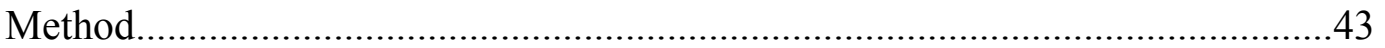

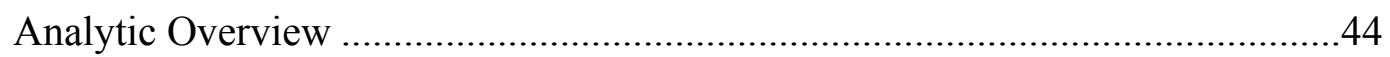

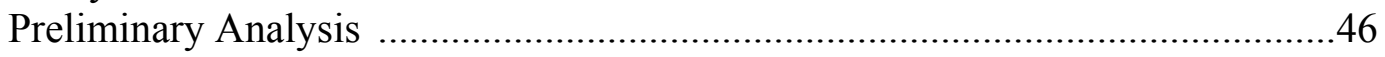

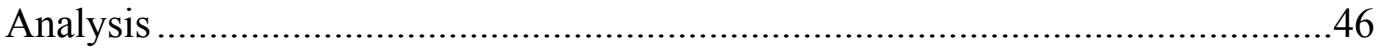

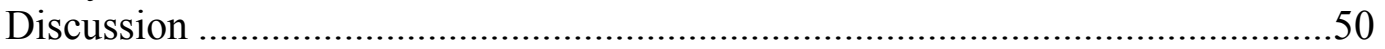

VI. STUDY II - ROLE OF EARLY SPATIAL ACTIVITY EXPERIENCE IN

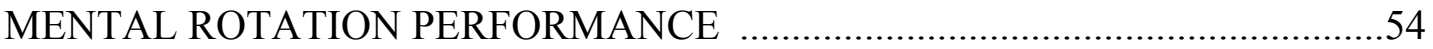

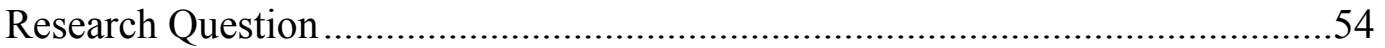

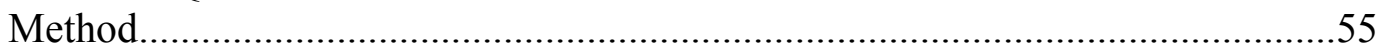

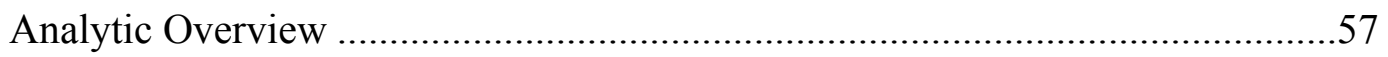

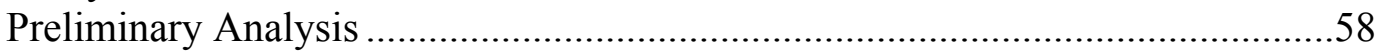

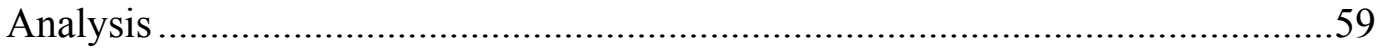

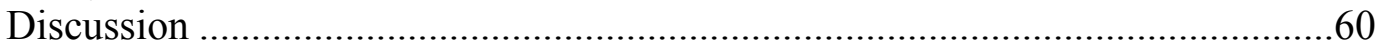




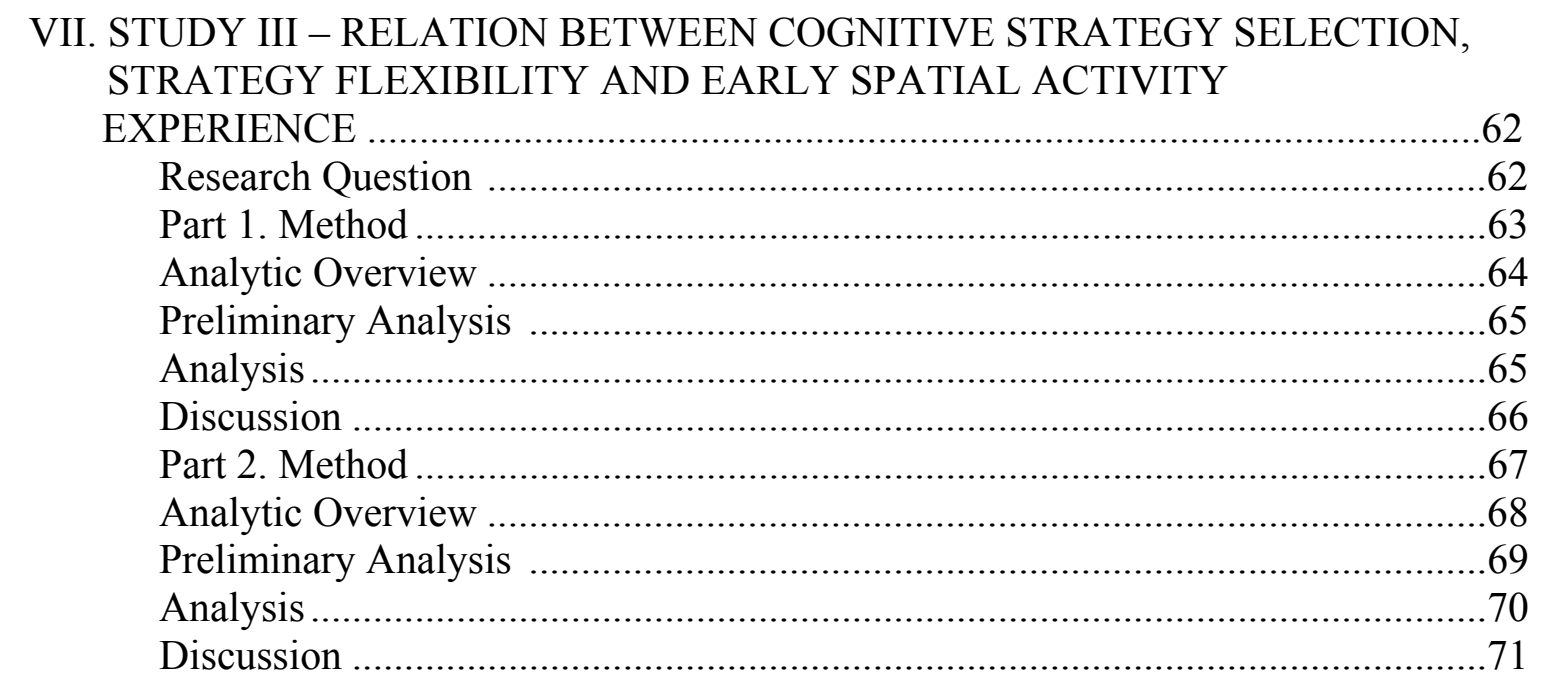

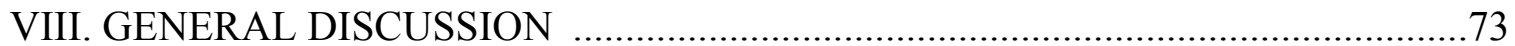

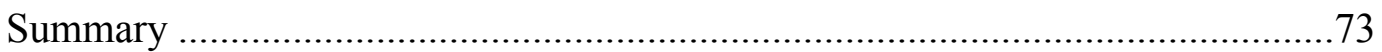

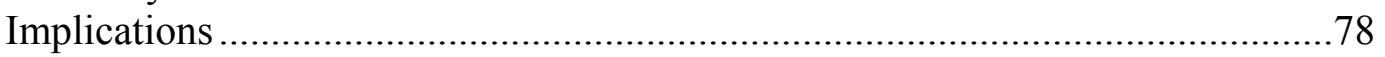

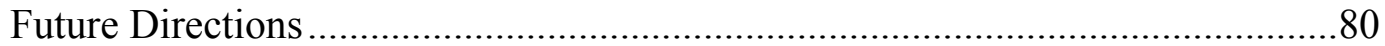

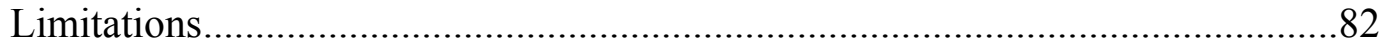

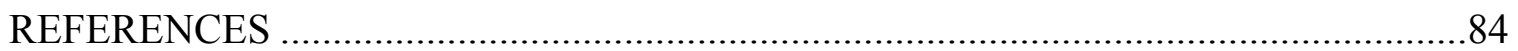

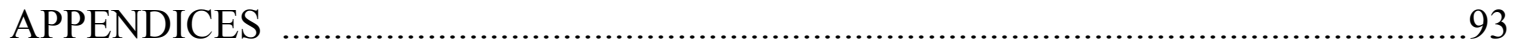

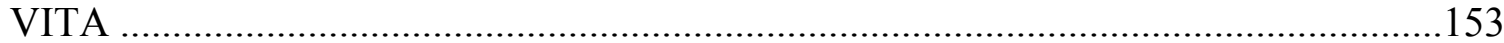




\section{LIST OF TABLES}

TABLE

PAGE

1. Summary Statistics of Participant Demographic Information for Study I and III ..........101

2. Coding Scheme for Video Protocol .................................................................... 102

3. Descriptive Statistics of Participant Demographic Information for Study I and III (1) .103

4. Descriptive Statistics for Study Variables ......................................................... 104

5. Comparison of Mental Rotation Performance .................................................... 105

6. Sex Differences in Mental Rotation Performance .................................................... 106

7. Relation between Self-reported (Video Protocol) Cognitive Strategy Selection and Mental Rotation Performance

8. Mean Estimates of Each Item in the Strategy Questionnaire .108

9. Correlations between Items in the Strategy Questionnaire.

10. Fit Indices for the Latent Profile Analysis Models of Cognitive Strategy Selection during Mental Rotation 110

11. Average Latent Class Probabilities for Most Likely Class Membership

12. Latent Class Means for Eye Movement Parameters in the 2-class Latent Model

13. Logistic regression Coefficients for the 2-class Latent Profile Analysis with Mental Rotation Score and Participant Sex as Latent Class Predictors.

14. Latent Classes of Cognitive Strategy by Participant Sex.

15. Summary Statistics of Participant Demographic Information for Study II and Study III (1)

16. Descriptive Statistics of Participant Demographic Information for Study II and Study III (1) 116

17. Summary Statistics of Participant Demographic Information for Study III (B)

18. Correlation between Cognitive Strategy Flexibility and Mental Rotation Performance 
19. Cognitive Strategy Flexibility by Participant Sex

20. Correlation between Cognitive Strategy Flexibility and Early Spatial Activity Experience 


\section{LIST OF FIGURES}

FIGURE

PAGE

1. A sample stimulus used in the monochromatic experimental condition. The two figures are similar and can be rotated into congruence. The correct participant response for this stimulus is match....

2. A sample stimulus used in the monochromatic experimental condition. The two figures are mirror images of each other and cannot be rotated into congruence. The correct participant response for this stimulus is non-match.

3. Overarching research question guiding the current dissertation: Do experiential factors affect adult mental rotation performance through the development of an optimum cognitive strategy?

4. Hypothesized eye-gaze pattern of a piecemeal strategy with a longer confirmation stage characterized by frequent peaks.

5. Hypothesized eye-gaze pattern of a holistic strategy with a longer transformation and comparison stage characterized by frequent plateaus

6. A sample stimulus used in the facilitating cue (FC) condition. The red cube in the left figure corresponds to the red cube in the right figure. Therefore, the non-spatial color information is hypothesized to facilitate mental rotation.

7. A sample stimulus used in the inhibiting cue (IC) condition. The red cube in the left figure does not correspond to the red cube in the right figure. Therefore, the non-spatial color information is hypothesized to inhibit mental rotation.

8. Flowchart of procedure and measures used in the current dissertation

9. Eye-tracking experimental room set-up

10. A sample stimulus used in the monochromatic condition. The red tinted box encompassing the figure indicates the area-of-interest (AOI) used to determine eye movement parameters

11. A sample stimulus used in the non-spatial cue condition. The red tinted box encompassing the red cube indicates the area-of-interest (AOI) used to determine eye movement parameters

12. Relation between mental rotation score and experimental condition (by participant sex) 
13. Relation between self-reported (verbal protocol) cognitive strategy selection and mental rotation performance

14. Relation between self-reported (verbal protocol) cognitive strategy selection and mental rotation performance for female participants only

15. Relation between self-reported (verbal protocol) cognitive strategy selection and mental rotation performance for male participants only

16. Relation between self-rated strategy items calculated as a proportion of holistic to piecemeal item ratings, and experimental condition (by participant sex)

17. Eye movement in the monochromatic condition depicting eye gaze path (indicated by numbers) and duration (indicated by size)

18. Eye movement in the facilitating cue (FC) condition depicting the use of the facilitating non-spatial information i.e. the red cube to enhance mental rotation

19. Eye movement in the inhibiting cue (IC) condition depicting the use of the inhibiting non-spatial information i.e. the red cube to inhibit mental rotation.

20. A 2-class latent profile model using mental rotation score (MRT) as a latent class predictor in the monochromatic condition

21. A 2-class latent profile model using mental rotation score (MRT) as a latent class predictor in the facilitating cue (FC) condition

22. Relation between latent classes and mental rotation performance in the monochromatic condition (by participant sex)

23. Relation between latent classes and mental rotation performance in the facilitating cue condition (by participant sex)

24. Sex differences in participation in male sex-typed spatial activity experience (by experimental condition)

25. Sex differences in participation in female sex-typed spatial activity experience (by experimental condition)

26. Scatterplot showing the relation between number of masculine spatial activities and MRT scores, after controlling for other spatial activities .146

27. Mediation analysis investigating the role of early spatial activity experience in mental rotation performance 
28. Relation between latent variable of cognitive strategy selection and early engagement in male sex-typed spatial activity experience

29. Sample plot of changepoints of two random participants to demonstrate PELT algorithm

30. Relation between cognitive strategy flexibility (indicated by the number of changes detected in eye gaze patterns) and mental rotation performance in the monochromatic condition

31. Relation between cognitive strategy flexibility (indicated by the number of changes detected in eye gaze patterns) and mental rotation performance in the facilitating cue (FC) condition..

32. Relation between cognitive strategy flexibility (indicated by the number of changes detected in eye gaze patterns) and mental rotation performance in the inhibiting cue (IC) condition.. 


\section{INTRODUCTION}

Mental rotation ability is the ability to mentally rotate 2-dimensional or 3dimensional objects in space. It involves the spatial representation, transformation and prediction of mental objects and their locations. It is a skill used in everyday tasks like rearranging furniture, fitting luggage in the trunk of a car or packing a picnic basket. The importance of mental rotation ability cannot be overemphasized. Mental rotation is the most fundamental spatial thinking task studied by cognitive psychologists (Hegarty, 2010). Spatial thinking has been found to be a skill embedded in many scientific domains (Hegarty, 2010). More specifically, research now suggests a link between spatial ability and success in science, technology, engineering and mathematics (STEM) disciplines (Lubinski, 2010; Wai, Lubinski, \& Benbow, 2009). Mental rotation abilities have been found to be significantly correlated to anatomy examination/learning results (Guillot, Champely, Batier, Thiriet, \& Collet, 2006; Hoyek et al., 2009). Spatial research using mental rotation tasks has helped further our understanding of imagery and the cognitive processes involved in mental representation (Pylyshyn, 2003; Shepard \& Metzler, 1971). It has also provided insight into inter-individual differences in cognitive strategy selection (Khooshabeh, Hegarty \& Shipley, 2012). The recent appreciation for the extent to which spatial thinking is central to educational and occupational settings (Lubinski, 2010) has led to a renewed interest in the field. The consistent sex differences favoring males, found in mental rotation literature raises an important concern regarding its implications for the representation and success of women in the STEM fields. However, the silver lining is research indicating that mental rotation ability can be enhanced with experience (Nazareth et al., 2013) and training (Wright et al., 2008) and that the effects of 
training can be transferred to other spatial tasks (Wright et al., 2008) and can be sustained for months (Terlecki et al., 2008).

Mental rotation ability is tested in a laboratory using tasks like the Shepard and Metzler (1971) mental rotation task. The Shepard and Metzler (1971) mental rotation task comprises pairs of figures of three dimensional, asymmetrical assemblies of cubes. In each pair, the figures are either identical assemblies of cubes but rotated at different angles from each other (see Figure 1) or assemblies of cubes, which are mirror images of each other and rotated at different angles (see Figure 2). In their seminal work, Shepard and Metzler (1971) demonstrated that the reaction time to mentally rotate objects is linearly related to the angle of rotation. They interpreted their findings to mean that mental rotation is similar to the physical manipulation of objects. For example, the amount of time required to physically rotate an object by 30 degrees would be less than the amount of time to rotate it by 120 degrees. The same rule has been found to apply to mental rotation. The analogy between physical rotation and mental rotation has made it possible to use varying degrees of rotation, accuracy and time-to-completion as measures of individual differences in mental rotation ability. However, to design effective spatial training techniques to reduce the inter-individual differences in mental rotation ability, we need to understand the cognitive mechanism underlying mental rotation ability. What are the underlying cognitive strategies selected by an individual to efficiently and accurately mentally rotate an object? When do we choose one cognitive strategy over the other? Is our preferential cognitive strategy our only cognitive strategy or are we capable of adapting depending on task demand? Do cognitive strategies develop as a consequence 
of experiential factors? The set of experiments in the current dissertation have been designed to address these research questions.

Cognitive strategy refers to an individual's preferential or habitual manner of processing information for problem solving (Kozhevnikov, 2007). One of the biggest problems with research in mental rotation strategies is the use of different terminology to define and describe similar cognitive processes. By definition, it would appear that mental rotation would involve visualization and transformation of mental images. Shepard and Metzler (1971) proposed a cognitive strategy describing a holistic analog process. However, subsequent research using mental rotation tasks like the Vandenberg and Kuse (1978) mental rotation task and the Shepard and Metzler (1971) mental rotation task has found other cognitive strategies involving task decomposition and rule-based reasoning. In Hegarty (2010), participants were found to use mental imagery strategies (imagining rotating the object or rotating themselves around the object), spatial analytic strategies (noting the directions of the different sections of the target, comparing end arms of the target images), pure analytic strategies (counting the number of cubes) and test taking strategies (skipping a question, guessing, etc.).

Similarly, Kozhevnikov, Hegarty, and Mayer (2002) discuss the relationship between performance on spatial tests and the visualizer-verbalizer cognitive styles. Visualizers were defined as individuals who rely on imagery processes when attempting to perform cognitive tasks. Verbalizers were individuals who preferred to process information by verbal-logical means. Thus, one may hypothesize that a visualizer would be more likely to use the mental imagery strategy while a verbalizer would be more likely to use spatial analytic and pure analytic strategies as described in Hegarty (2010). 
Khooshabeh et al. (2012) describe mental rotation strategies in terms of a holistic or piecemeal approach to mental rotation. They define a holistic strategy as one in which an individual mentally rotates an image as a whole i.e., encoding the complete spatial information of the presented image. A piecemeal strategy, on the other hand, involves the breaking down of the image into several pieces for rotation and comparison. Comparing the different cognitive strategies, a mental imagery strategy could be considered similar to a holistic strategy, whereas spatial or pure analytic strategy could be considered similar to a piecemeal strategy. Similarly, visualizers would be more likely to use a holistic strategy as compared to verbalizers who may choose to use a piecemeal approach. The above discussion is meant to highlight the considerable overlap between different cognitive strategies, which tend to define and describe similar cognitive processes. In this dissertation, the terms holistic and piecemeal will be used to describe the cognitive strategies as they are most frequently used with regard to mental rotation strategies.

Most research on cognitive strategy relies on participant self-report for strategy information (Gluck \& Fitting, 2003; Moe,Meneghetti \& Cadinu, 2008; Schultz, 1991). Self-report of cognitive processes may require a conscious awareness of the different strategies being used with different stimuli, which may not always be possible. Similarly, checklists or cognitive strategy/styles questionnaires are limited by their suggestive nature (Hegarty, 2010). These methods are vulnerable to participant interpretation of cognitive strategies and processes used during mental rotation. To overcome this drawback, the set of experiments in the current dissertation uses eye-tracking technology 
in addition to self-report verbal descriptions and a cognitive strategy questionnaire to examine cognitive strategy selection.

Plugging the gap. The overarching developmental question guiding the current dissertation is - Do experiential factors affect adult mental rotation performance through the development of an optimum cognitive strategy? (see Figure 3). However, a first step towards that lofty goal is to understand cognitive strategy selection as a mechanism of inter-individual differences in mental rotation performance. An important goal of the current dissertation was to identify the different cognitive strategies used in mental rotation and to investigate their relation to mental rotation performance. A second aim was to set the foundation for the argument that cognitive strategy selection is the mechanism that develops as a consequence of spatial activity experience and thus establish a link between cognitive and experiential factors that affect adult mental rotation performance. However, it is only the first step towards understanding the effect of experiential factors on the development of cognitive strategy selection.

The set of studies included in the current dissertation draws inferences from comparisons made between three experimental conditions. The three conditions differ in the amount and type of non-spatial information present in the task stimuli. Study I investigates the different types of cognitive strategies selected during mental rotation, where eye movement pattern is used as an indicator of the underlying cognitive strategy. Study II examines the role of early spatial activity experience in mental rotation performance. Finally, Study III examines the developmental role of early spatial activity experience in the selection of an optimum cognitive strategy and strategy flexibility to enhance mental rotation performance. Since eye fixations have been shown to be closely 
linked to the human information processing system (Just \& Carpenter, 1976), all three studies draw inferences about cognitive strategy selection using the participants' eye gaze pattern measured with a Tobii X60 eye tracker. 


\section{LITERATURE REVIEW}

The illusive quest to understand completely the cognitive processes involved in accessing, storing and manipulating different types of information has led to the design and use of different cognitive tasks, not the least of which is the Shepard and Metzler (1971) mental rotation task. More recently it has been used as a fundamental task in spatial cognition research. Spatial literature reports a consistent sex difference with males outperforming females on mental rotation tasks, like the Vandenberg and Kuse (1978) adaptation of the Shepard and Metzler (1971) mental rotation task (D. Voyer et al., 1995). Naturally, these findings have raised questions about the factors responsible for the gender disparity in spatial performance. With research now suggesting a link between spatial ability and success in science, technology, engineering and mathematics (STEM) disciplines (Lubinski, 2010; Wai, et al., 2009) the need to reduce this sex difference has become more urgent. Research suggests a mediating role of spatial activity experience in reducing sex differences in mental rotation performance (Nazareth et al., 2013). However, some questions still need answering to understand the role of experiential factors in mental rotation. For example, if indeed spatial activity experience is predictive of mental rotation performance, what cognitive mechanism is being developed as a consequence of these experiential factors?

\section{Spatial Ability}

Linn and Petersen (1985) define spatial ability as the ability to generate, retain, retrieve and transform well-structured visual images. Spatial ability is an overarching cognitive construct that comprises three distinct skills. These are spatial perception, mental rotation ability and spatial visualization. Spatial perception requires an individual 
to determine spatial relationships with respect to the orientation of his/her body, despite the presence of distracting information. Mental rotation is the ability of an individual to mentally rotate two- or three- dimensional objects in space. Spatial visualization involves multistep manipulations of spatially presented information and thus may involve a combination of spatial perception and mental rotation (Linn \& Petersen, 1985). While sex differences have been observed in all forms of spatial tasks, the most consistent sex differences have been found in mental rotation studies. These studies also report the largest effect sizes for sex differences reported in the spatial literature (Hyde, 1990). Therefore, for the purpose of the current dissertation research, participants have been tested on a mental rotation task, namely the Shepard and Metzler (1971) mental rotation test. However, there is no reason to believe that the principles used here to test for strategy-selection and manipulation of strategy-selection cannot be used for tasks involving spatial perception and spatial visualization.

\section{Mental Rotation Ability}

Mental rotation is defined as the ability to rotate two- and three- dimensional objects in space. It is considered the most fundamental skill in spatial development. Mental rotation has been studied for the last three decades and while the Shepard and Metzler (1971) mental rotation task and its adaptations like the Vandenberg and Kuse (1978) mental rotation test have been the most widely used thus far, findings have been replicated with stimuli that are very different from the assembled cube design. Mental rotation research is not limited to spatial cognition alone, but has far larger theoretical implications. Mental rotation processes are often referred to on the debate on mental imagery (Pylyshyn, 2003). In their seminal study, Shepard and Metzler (1971) found that 
the response time of participants to mentally rotate images was linearly related to the size of the angle of rotation. Their finding was used to support the analog representation hypothesis that argued for a "pictorial" cognitive representation of images. If the response time was indeed positively correlated to the angle of rotation, it implied that the mental rotation process was a mental simulation of the physical rotation process in the real world. Mental rotation tasks were used in subsequent studies to understand the human information processing system i.e., cognitive strategies involved in manipulating mental images (Khooshabeh et al., 2012).

Sex Differences: The Role of Experiential Factors. Research shows a consistent sex difference in mental rotation ability (D. Voyer et al., 1995). Although there have been speculations about genetic and hormonal reasons for the difference in male and female spatial ability, research has been largely inconclusive (Alington, Leaf \& Monaghan, 1992). In addition to these biological factors, there is a large body of spatial research that examines experiential factors as a possible explanation for sex differences in mental rotation ability. These experiential factors range from exposure to visuospatial activities (Baenninger \& Newcombe, 1989), to videogame experience and training (Cherney, 2008; Moreau, 2013; Terlecki et al., 2008; Terlecki \& Newcombe, 2005), to map reading and navigation (Cherney, 2008), to sports (Cherney, 2001), to years in mathematics (Lippa, Collaer, \& Peters, 2009) and to music training (Ginn \& Pickens, 2005) among others. Children spend a large proportion of time in gendered activities that differ in the extent of spatial content (Cherney \& Voyer, 2010). Overall boys have been found to engage in significantly more activities linked to the development of spatial skills like video gaming and sports (Cherney \& London, 2006). D.Voyer, Nolan, and Voyer (2000) found that 
sports preference was a significant predictor of male spatial ability while toys preference was a significant predictor of female spatial ability. The findings of Cherney and Voyer (2010) suggest that males likely spend more time in male sex-typed spatial activities while females are more likely to engage in feminine sex-typed non-spatial activities. To examine the role of different spatial activities in the development of spatial skill and to investigate the extent to which sex-typing causes differential engagement in these activities for both sexes, Newcombe, Bandura and Taylor (1983), developed the spatial activities experience questionnaire. They found a significant correlation between spatial ability as measured by the Differential Aptitude Test (DAT) and spatial activity experience. In similar research, Nazareth et al. (2013) discuss the mediating role of spatial activity experience in reducing sex differences in mental rotation performance. They found that the relationship between sex of the participant and their score on the Shepard and Metzler (1971) mental rotation task was partially mediated by the number of male/masculine sex-typed spatial activities participants had engaged in as youth. Similar research using abbreviated or adapted versions of the spatial activity experience questionnaire (Newcombe et al., 1983) has found a positive correlation between spatial activity experience and performance on spatial tasks (Quaiser-Pohl \& Lehmann, 2010; Signorella, Krupa, Jamison, \& Lyons, 1986). Similarly, Cherney (2006) found that exposure to computer games improved mental rotation scores with improvement for women being significantly greater than that for men. Their findings suggest that even minimal exposure to video/computer games can help improve mental rotation performance. Thus, although research has consistently shown sex differences on spatial tasks, it has also suggested that mental rotation ability can be enhanced with experience 
(Nazareth et al., 2013; Terlecki et al., 2008) and training (Wright et al., 2008) and the

effects of training are found to be transferable to other spatial tasks (Wright et al., 2008) and is sustainable for months (Terlecki et al., 2008). Spatial malleability raises some interesting questions, not the least of which is, what develops as a result of experiential factors ultimately enhancing mental rotation ability?

\section{Cognitive Strategy}

One plausible explanation for the role of experiential factors on mental rotation performance is the development of an optimum cognitive strategy. Cognitive strategy refers to an individual's preferential or habitual manner of processing information for problem solving (Kozhevnikov, 2007). Spatial ability comprises three distinct spatial skills i.e., spatial perception, mental rotation and spatial visualization. By definition it can be hypothesized that the cognitive processes involved in each of these spatial categories would be different. Consequently, the type of strategy used would largely depend on the demands of the task, i.e., the stimuli used to study these processes. For mental rotation tasks, holistic and piecemeal cognitive strategies are two of the most frequently studied strategies (Khooshabeh et al., 2012). Research suggests that individuals with good rotation ability show a preference for holistic strategies while those with poor rotation ability show a preference for piecemeal analytic strategies (Khooshabeh \& Hegarty, 2010). A holistic strategy involves mentally rotating an image as a whole i.e., encoding the complete spatial information of the presented image. A piecemeal strategy, on the other hand, involves the breaking down of the image into several pieces i.e., encoding partial spatial information of the presented image. In the piecemeal strategy, an individual may mentally rotate one piece in congruence with the comparison figure and then apply 
the same rotation to the other parts of the figure to see if they match (Khooshabeh et al., 2012). There is evidence to suggest that men and women tend to use different cognitive strategies for solving spatial tasks.

Research on mental rotation is biased towards a holistic strategy as the optimum cognitive strategy. Studies have found that participants who show a preference for the holistic strategy tend to perform better in mental rotation tasks as compared to participants who show a preference for the piecemeal strategy (Khooshabeh et al., 2012). Research on spatial intelligence, however, may be drawing an altogether different picture. Hegarty (2010) defines spatial intelligence as adaptive spatial thinking. Mental rotation tasks (e.g., Vandenberg and Kuse [1978] MRT; paper folding) are simplistic versions of the real world and do not fully capture the spatial intelligence required for complex domains like mechanical engineering, medicine and architecture, to name a few. While new technologies may have been designed to support spatial thinking, their use, in turn demands different types of spatial thinking. Hegarty (2010) argues that mental imagery or mental simulation is augmented by analytic strategies like task decomposition and rulebased reasoning. Task decomposition was observed in Hegarty (1992) where complex tasks like inferring the motion of a complex pulley mechanism, was solved by decomposing the task into a sequence of step-wise interactions. Rule-based learning was observed in Schwartz (1996) where participants discovered rules about gear movement while solving similar stimuli but reverted to holistic mental simulation when shown a novel gear stimuli. Stieff (2007) found that novice students used a holistic mental rotation strategy when determining if two 3D structures of molecules were similar or mirror images as compared to expert chemists who were more likely to use an analytical 
strategy. Thus, research on complex spatial stimuli has found that visualization (similar to holistic) strategies are used by novices or experts in novel situations whereas rule-based analytic strategies are used by experts or novices who have been taught through instruction (Hegarty, 2010; Schwartz, 1996; Stieff, 2007).The experience-dependent strategy selection suggests that the holistic strategy may not be the optimum spatial strategy but is just one spatial strategy among a number of available strategies that a person must choose from depending upon the demands of the task. In fact, for most real world spatial tasks, an individual should be able to augment a holistic strategy with a rule-based piecemeal-like analytic strategy. If past experience is a criterion for preferential cognitive strategies, then the best performer should be the one who switches between a holistic and piecemeal strategy depending upon the demands of the task. This highlights a different aspect of cognitive strategy selection in mental rotation i.e. strategy flexibility.

\section{A Neuroscience Perspective}

Mental rotation findings have been well replicated in behavioral studies.

However, it is important to understand the neural structures that support and limit this observed behavior. Recent neuroimaging studies using mental rotation tasks have shown that poor- and vivid- imagers activate different brain regions while performing the same tasks (Logie, Pernet, Buonocore, \& Sala, 2011) providing further evidence that different cognitive strategies are used to solve the same spatial tasks. Clements-Stephens, Rimrodt and Cutting (2009) found that even when matched for performance, sex differences existed for brain network activation related to visuospatial processing. Similarly, Jordan, Wüstenberg, Heinze, Peters, and Jäncke (2002) found that there were significant sex 
differences in cortical activation with men showing a significant additional activation of the left motor cortex, further corroborating the story painted by behavioral studies of spatial processing that sex differences in mental rotation may be attributed to differences in cognitive strategy selection. The main areas that have been linked with mental rotation are the parietal areas, occipital, temporal i.e., premotor and somatomotor and the basal ganglia (Ark, 2002). However, given that mental rotation is a highly distributed processing task, there is much debate about the extent to which the different brain regions are involved.

\section{Eye-tracking}

The set of experiments in the current dissertation will be conducted and analyzed using a Tobii X60 eye-tracker. An important question to address at this point is - why track eye movement? How does eye movement help us access the complex neural mechanism underlying the cognitive process? With eye tracking, we are capturing the participant's path of attention. In other words, we capture those components of the image, which the individual deemed to be attention-worthy, and the order in which the different components captured the participant's eye movement. In focusing on a particular component of the image, an individual is choosing to block out other distractions because of limitations in the human information processing capacity (Duchowski, 2007). Eye fixations are therefore closely linked to the human information processing system. One of the biggest advantages to recording and analyzing eye-gaze data is that tasks can be decomposed into stages of different processing durations to capture the underlying cognitive processes. There are several eye-tracking specific terms used throughout this dissertation, the definitions for which have been included in Appendix A. 
Just and Carpenter (1976) examined the relationship between eye fixations and cognitive processes and their findings suggest that cognitive processes during mental rotation tasks could be categorized into (1) search i.e., the process to select similar segments between the two figures that can be transformed into each other (2) transformation and comparison i.e., the process of stepwise reorientation of figure segments and comparison between the reoriented or transformed figures and (3) confirmation i.e., process of checking if the remaining segments of the two figures are congruent. In the study on mental rotation strategy, gaze patterns of each individual for each stimulus can then be measured against the baseline hypothesized gaze-pattern mimicking this three-stage cognitive strategy. One might expect frequent saccades or peaks in the first portion of the gaze-pattern for the search stage, followed by longer fixation duration or plateaus in the gaze-pattern for the transformation-comparison stage, only to end once again with frequent saccades or peaks for the confirmation stage. This 3stage model appears to be a combination of both piecemeal as well as holistic strategies. It is plausible that the two strategies, then, differ not by stage (pattern) but in the duration of each stage (length of pattern). Thus, a piecemeal strategy would have a long stage 3 i.e., confirmation characterized by peaks (see Figure 4) whereas a holistic strategy would have a longer stage 2 i.e., transformation and comparison characterized by plateaus (see Figure 5). A holistic strategy selection may mean that an individual does not enter the confirmation stage, as there is no image break-up by segments. Eye- gaze data could then be grouped on pattern-similarity and examined for sex differences and performance differences. 
Although the use of eye fixation as a measure of cognitive strategies dates back to the 1800 s, the accuracy of the different processes used for obtaining gaze data is questionable. Recently, advanced eye-tracking equipment and software have allowed a definitive move towards accurate eye fixation information. In order to find individual differences in gaze patterns, raw eye-movement data are processed into fixations. Fixations, although thought of as a particular eye-pattern, is mostly just a mathematical and statistical process of parsing data to describe eye-movement variants. Eye-tracking software uses different fixation filters to set statistical rules for parsing data. Hence, filter specifications can strongly affect how gaze-plots, heat maps, eye-tracking metrics, clusters, etc. are defined, analyzed and interpreted. Eye-trackers have a high sampling rate of $60-120 \mathrm{~Hz}$, and come with built-in fixation algorithms which makes for simplistic operations for laboratory research, allowing flexibility of fixation filter specifications to suit different research questions. However, there seems to be inconsistent interpretations of the same parameters. Although eye-movement studies have been recorded from over a century ago, there is still no clear understanding on which aspect of eye-movement can be construed to represent cognition (Aslin, 2012). One solution, as proposed by $\mathrm{Yu}$, Yurovsky, and $\mathrm{Xu}$ (2011) is for the experimenter to 'look' at the raw data and to spot significant gaze events. Although there is definitely some merit to their suggestion, looking at data should act as a preliminary test for any type of data collection, not just eye-tracking data. Moreover, looking at datasets to spot abnormalities may work with smaller datasets but not for eye-tracking studies that generate thousands of time points, coordinate points and fixation points per participant. Another potential problem with large datasets generated by eye-trackers is that statistical analysis is bound to return 
significant relationships and lead the researcher astray if there are no strong apriori theoretical underpinnings to the variables used for the analysis. Two additional statistical strategies that have been suggested are (1) the use of cross-validation techniques to iteratively withhold some of the data as a test of hypotheses that were discovered with the remaining data and (2) hierarchical regression techniques to determine which of the myriad of coded variables matter (and by how much) (Aslin, 2012). A valid point made by both is that measures that are correlated determined by changes in stimuli may not be an appropriate measure of the cognitive processes underlying how the stimuli are processed. For example, one may interpret a greater number of fixations on one object to imply that the participant is using a mental strategy to rotate the image and hence takes longer to switch between objects. One the other hand, one may interpret a greater number of fixations on one object to imply that the individual is using a piecemeal strategy to compare each leg of the figure to its corresponding leg in the other figure. Thus, the exact same description of the fixation parameter can be used to justify a piecemeal strategy as well as a holistic strategy. Similarly other gaze parameters generated by the eye-tracker can be used to justify either strategy or to define a third one. Some researchers have indicated that if the number of fixations equals the number of saccades that would indicate a holistic strategy (Khooshabeh \& Hegarty, 2010). The rationale here is that a participant fixates on an entire figure and then shifts to compare it with the other figure. However, one can argue that the number of visits (made up of several fixations) in each figure should be equal to the number of saccades across figures using the same rationale. Thus, gaze parameters need to be understood in relation to the figure, as well as in relation to each other. How does one go about deciding the appropriate gaze parameter as 
an indicator of a cognitive strategy? Since eye-tracking research is still at such a nascent stage, a good idea would be to use exploratory analysis on different eye-tracking parameters for different sub-groups of individuals (i.e., males and females, high- and low- performers, high - and low- early spatial experience, self-report holistic and selfreport piecemeal, etc.) The experiments in the present dissertation use the Tobii Studio's default I-VT (velocity threshold identification) fixation filter settings for all fixation analyses and uses raw data (no fixation filters) for individual gaze-plots. The different fixation settings ensure that findings are presented in two formats (i) the raw eye-gaze data that give a visual representation and is free of fixation filter constraints (ii) the standard fixation parameters that tend to be used in eye-tracking research but are subject to filter constraints.

\section{Research Aims}

The current dissertation aimed to answer the question - Do early spatial activity experiences affect adult mental rotation performance through the development of an optimum cognitive strategy. There were three experimental conditions that differed in the amount and type of non-spatial information presented in the stimuli. In the monochromatic experimental condition, participants viewed paired figures of assembled cubes based on the Shepard and Metzler (1971) mental rotation task (see Figures 1 \& 2). In the facilitating cue (FC) condition, paired figures contained corresponding red cubes that acted as non-spatial information facilitating mental rotation (see Figure 6). In the inhibiting cue (IC) condition, paired figures contained asynchronous red cubes that acted as non-spatial information inhibiting mental rotation (see Figure 7). 
Study I Research Questions. What are the different types of cognitive strategies selected during mental rotation? Do these strategies change with the amount and type of non-spatial information available in the stimulus? Is one strategy more effective than the others? Are there sex differences in cognitive strategy selection?

It was hypothesized that there would be two distinct eye movement patterns indicative of the different cognitive strategies selected in the monochromatic condition. An eye pattern similar to a holistic strategy (i.e. high fixation counts, high fixation duration and low visit counts) would emerge as a better cognitive strategy as compared to an eye pattern similar to a piecemeal strategy (i.e. high fixation counts, low fixation duration and high visit counts). The two distinct eye patterns would emerge irrespective of the type of non-spatial information presented to the participant. However, in the facilitating cue (FC) condition, an eye pattern indicative of a participant's inclusion of the facilitating red cube during mental rotation would emerge as the optimum cognitive strategy. In contrast, in the inhibiting cue (IC) condition, an eye pattern indicative of a participant's exclusion of the inhibiting red cube during mental rotation would emerge as the optimum cognitive strategy. Thus the three experimental conditions were designed to not only give insight into cognitive strategy selection but also strategy flexibility based on facilitating or inhibiting information. Given that males consistently outperform females on mental rotation tasks, it was hypothesized that the proportion of males selecting the optimum cognitive strategy would be significantly higher than that of females in each experimental condition.

Study II Research Questions. Do early spatial activity experiences affect adult mental rotation performance? Do certain types of spatial activities enhance adult mental 
rotation performance more than others? Do spatial activity experiences mediate sex differences in mental rotation performance?

It was hypothesized that early spatial activity experience would be significantly correlated with mental rotation performance i.e., participants who had engaged in spatial activities as youth would score significantly higher on the mental rotation task as compared to participants who had not engaged in similar spatial activities independent of the participant's experimental condition. Given the existing sex difference in mental rotation, in favor of males and the greater likelihood of male participation in masculine/male sex-typed spatial activities, it was hypothesized that male sex-typed and not female sex-typed spatial activities would be significantly associated with higher mental rotation performance. Finally, it was hypothesized that male sex-typed spatial activities would partially mediate the relation between participant sex and mental rotation performance.

Study III Research Question. Do early spatial activity experiences play a role in the development of an optimum cognitive strategy? Does strategy flexibility enhance mental rotation performance? Are early spatial activity experiences indicative of strategy flexibility?

It was hypothesized that the latent variable of cognitive strategy selection would significantly predict early spatial activity experience in all three experimental conditions. However, in addition to cognitive strategy selection, the facilitating cue (FC) and inhibiting cue (IC) non-spatial conditions required strategy flexibility in order to incorporate or suppress non-spatial information. There is very little research investigating the relation between strategy flexibility and early spatial activity experience. It was 
hypothesized that there would be a positive association between early spatial activity experience, especially male sex-typed spatial activities and strategy flexibility.

Additionally, it was hypothesized that male participants would demonstrate significantly more strategy flexibility as compared to female participants. 


\section{METHODOLOGY}

\section{Participants}

Adult university students enrolled in undergraduate psychology courses, between the ages of 18-25 were recruited from a university in the Southeastern United States. The university-wide online recruitment website SONA SYSTEMS was used to get undergraduate students to enroll for the study. Students were granted one SONA credit for participation in the study that could be used as extra credit. The final sample consisted of 387 unique participants across three experimental conditions (Mage $=20.72$ years, $S D=$ 2.42 years; 109 male, 278 female). Participants were randomly assigned to three experimental conditions depending on the amount and type of non-spatial information presented during the mental rotation task. There were 164 participants in the monochromatic condition (52 male, 112 female), 93 participants in the facilitating cue (FC) condition (20 male, 73 female) and 130 participants in the inhibiting cue (IC) condition (37 male, 93 female) respectively. Participants represented an ethnically (67.7\% Hispanic/Spanish/Latino) and racially (61.83\% White; 18.97\% Black; 7.13\% Asian and $12.67 \%$ other/mixed-race) diverse group. The summary statistics of participant demographic information for each experimental condition are presented in Table 1.

\section{Materials}

Hardware and Software Specifications. A standard Dell desktop with $2.80 \mathrm{GHz}$ processor and $8 \mathrm{~GB}$ of RAM was used to present the stimuli. A Tobii X60 eye tracker was used to record participant gaze data. The technical specifications of the Tobii X60 eye tracker are presented in Appendix A. The 40 stimuli per experimental condition were 
presented on a Sony Bravia (model number: KDL-46EX500) flat screen TV monitor using Tobii Studio 3.2, an eye tracking analysis and visualization software compatible with Tobii X60 eye trackers. A Sony Handicam (model number: HDR XR160) was used to record participant self-reports on task completion. A standard Dell Desktop with 3.40 GHz processor and $6 \mathrm{~GB}$ of RAM was used to analyze the Tobii recordings. Data were analyzed using statistical software MPLUS v7, R and SPSS v20.

Stimuli Description. Each stimulus consisted of two figures of 3-D assembled cubes used by Shepard and Metzler (1971) (see Figures $1 \& 2$ ), but had been adapted for the purposes of the current dissertation (see Figures $6 \& 7$ ). In the match stimuli, figures were paired such that they differed only in angular disparity and could be rotated into congruence (see Figure 1). In the non-match stimuli, figures were paired with their mirror images such that they could not be rotated into congruence (see Figure 2). There were 20 unique pairs of match stimuli and 20 of non-match stimuli for a total of 40 stimuli in each experimental condition.

In addition to the monochromatic figures, one of the cube faces was colored red for two of the three experimental conditions. In the facilitating cue (FC) non-spatial condition, color-cube figures (see Figure 6) were designed in a way that the red cubes were in the same position in the two figures to be compared. In the inhibiting cue (IC) non-spatial condition, color-cube figures (see Figure 7) were designed in a way that the red cubes were in different positions in the two figures to be compared. The coloring protocol for both the facilitating and inhibiting color-cube figures was predetermined to be completely random such that there was no one arm or cube that was specifically 
chosen to be the color cube in a stimulus. Thus, each facilitating and inhibiting colorcube stimuli was unique.

The stimuli were digitized, modified to include a red cube and made compatible with Tobii Studio using Adobe Premier Pro CS6.

Counterbalancing. In order to counterbalance for practice effects and fatigue, the 40 stimuli were divided into two sets of 20 stimuli each. The two sets of 20 stimuli were alternately presented at the beginning of a test condition. Stimuli within each set were not counterbalanced.

\section{Procedure}

Figure 8 shows a detailed flowchart of the different study components, and measures used for the current dissertation.

Step 1. Recruitment. Participants were recruited from a Southeastern University using the online recruitment website SONA SYSTEMS. The online recruitment advertisement informed participants that they would be required to complete a spatial test in a laboratory setting followed by an online survey. They were also told that the entire study duration would be approximately one hour. Participants would receive 1 SONA credit for the completion of the study, which could be used as extra credit for a psychology course. Participants between the ages of 18 and 25 years could sign up to participate in the study. This was the only eligibility criterion set for the online recruitment sign-up form.

Step 2. Informed Consent. Participants were asked to carefully read and sign an adult consent form pre-approved by the Institutional Review Board (IRB) at the beginning of their laboratory visit. The consent form informed participants that the 
purpose of the study was to examine the variables most effective in improving spatial skills. A trained research assistant described the study procedure in detail. Participants were reminded that their participation in the study was completely voluntary and that they could choose to withdraw from the study at any time during the course of the experiment. The process of consenting took on average 10-12 minutes.

Step 3. Eye tracking Study Component. If a participant agreed to participate in the study after reading the study information on the consent form and listening to the study description by the trained research assistant, they were asked to enter the eye tracking room (see Figure 9). The eye-tracker room consisted of a desk-mounted Tobii X60 eye tracker, a large screen TV monitor (model specification: Sony Bravia, KDL46EX500), and a video camera (model specification: Sony Handicam HDR XR160). Participants were asked to keep all their belongings (e.g., book bags, mobile phones, etc.) on a table at the end of the room so as to remove all distractions during the task.

Calibration. At the beginning of the mental rotation task, participants were taken through a calibration process. A calibration process is a quality control measure to ensure that the eye-tracker saves the specific characteristics of the participant's eyes. In Tobii $\mathrm{X} 60$, the participant eye characteristics are combined with an internal, physiological 3D eye model, which contains important information about shapes, light refraction and reflection of the cornea, etc. The combination of participant calibration and the Tobii internal 3D eye model is then used for all future gaze data statistics. Hence, the quality of participant calibration determines the accuracy of the participant gaze data produced by Tobii Studio. Participant calibration is a critical first step of the eye tracking study component. During the calibration process, participants were asked to closely follow a 
green dot that appeared to randomly move across the display screen allowing the eye tracker to record participant eye characteristics. A similar calibrating process was used on completion of the eye-tracking task.

Experimental conditions. On successful calibration, participants were randomly assigned to one of three experimental conditions that differed in the amount and type of non-spatial information present in the stimuli.

Monochromatic Condition. Participants were presented with two 3D figures of assembled cubes based on the Shepard and Metzler (1971) mental rotation task (see Figures $1 \& 2)$.

Facilitating Cue (FC) Condition. Participants were presented with two 3D figures of assembled cubes similar to that in the monochromatic condition. However, in this condition, figures were modified to include a non-spatial component i.e., one of the cubes in each figure was colored red. In the FC condition, the red cube in the left figure corresponded to the red cube in the right figure and thus acted as non-spatial information meant to facilitate or enhance mental rotation (see Figure 6).

Inhibiting Cue (IC) Condition. Similar to the FC condition, participants were presented with images containing a non-spatial component i.e., one of the cubes in each figure was colored red. However, in the IC condition, the red cube in the right figure did not correspond to the red cube in the left figure and thus acted as non-spatial information meant to distract or inhibit mental rotation (see Figure 7).

In all three experimental conditions stimuli were presented on a large television screen connected to a Tobii X60 eye-tracker. The eye-tracker was used to record participant gaze patterns as a measure of cognitive strategy selection. Participants were 
asked to decide if the two images were a match (similar but rotated) or a non-match (i.e. mirror images of each other). They were shown three solved trials as well as three practice trials before the task began. Participants received feedback for their responses to the practice trials. There were 40 trials with three rest periods of 30 seconds each after every 10 trials. Participants recorded their answers using a mouse connected to the recording computer. The task took approximately 20-25 minutes depending upon the participant's response time. The participant gaze data recorded during this phase were used as a primary measure of cognitive strategy selection. The eye tracking study component took on average 20-25 for completion from calibration at the beginning of the task to calibration at the end of the task.

Step 4. Verbal Protocol Study Component. At the end of the eye-tracking task, participants were asked a series of questions related to the mental rotation task completed during the eye tracking study component (See Appendix B). Participant response was recorded using a Sony Handicam. The verbal descriptions recorded during this phase was later coded and used as a secondary measure of cognitive strategy selection. The verbal protocol study component took on average 5-8 minutes for completion.

Step 5. Online Survey Study Component. Finally, each participant was asked to fill out an online survey, which included demographic, early spatial activity experience and strategy-selection information. The online survey study component took on average 15-20 minutes for completion. Appendix B includes the measures used in the online survey study component. 


\section{Measures}

Participants viewed the measures in the online survey in a fixed sequence:

Demographics, spatial anxiety scale, strategy questionnaire, spatial activity experience, recalled childhood gender identity/gender role questionnaire. A copy of the different measures used in the online survey is presented in Appendix B.

Shepard and Metzler (1971) Mental Rotation Task. The Shepard and Metzler (1971) mental rotation task comprises pairs of images of three dimensional, asymmetrical assemblies of cubes. In each pair, the images are either (1) identical assemblies of cubes but rotated at different angles from each other (see Figure 1) or (2) assemblies of cubes that are mirror images of each other and rotated at different angles (see Figure 2). Participants were shown 40 trials of matched and non-matched images. Items were counterbalanced for degree of rotation as well as matched and non-matched images. The facilitating cue (FC) and inhibiting cue (IC) experimental conditions testing for the effects of non-spatial information (i.e. color), consisted of modified stimuli that had one colored cube in each of the two images per pair (see Figures $6 \& 7$ ). Participants were asked to decide on each trial if the two images were a match (identical but rotated) or a non-match (mirror images).

The Shepard and Metzler (1971) mental rotation task was used as a measure of mental rotation ability.

Spatial Anxiety Scale. Lawton (1994) devised a spatial anxiety scale to measure the level of anxiety experienced by a participant in eight situations requiring spatial/navigational skills. For the purpose of the dissertation, a revised version published by Lawton \& Kallai (2002) was used. Participants were asked to rate how much they 
were bothered by a way-finding task that might produce anxiety, on a Likert scale ranging from ' 1 ' $=$ 'not at all' to ' 4 ' = 'Severely $-\mathrm{I}$ could barely stand it'. The Cronbach alpha for this scale was .87. An example question on the spatial anxiety scale is - " Deciding which direction to walk in an unfamiliar city or town after coming out of a train/bus/metro station or parking garage." The complete spatial anxiety scale is presented in Appendix B.

The spatial anxiety measure was included in the online survey so as to eliminate spatial anxiety as a possible confounding variable for performance differences across the three experimental conditions.

Strategy questionnaire. The online survey consisted of questions on strategy using a strategy questionnaire devised by Moè, Meneghetti, and Cadinu (2009a). The questionnaire aimed at assessing the strategies used to solve mental rotation task items. Questions were divided to test for a holistic strategy - "I rotated the stimulus on the left to match the stimulus on the right", "I rotated the stimulus on the right to match the stimulus on the left", "I rotated the stimulus as a single whole object" and "I imagined myself rotating around the object" and a piecemeal strategy - "I counted the cubes" and "I rotated the stimulus piece by piece." In all, there were six items on strategy. Participants were asked to rate how much they used each strategy, on a Likert scale ranging from ' 1 ' = 'not at all' to ' 7 ' = 'very much'. The strategy questionnaire had a low Cronbach alpha of -.28. A low Cronbach's alpha is desirable in this case, since we do not want the strategies to be related to each other. The complete strategy questionnaire is presented in Appendix B. 
The strategy questionnaire was included in the online survey to corroborate evidence of a particular cognitive strategy selected during the mental rotation task. Although eye movement parameters were used as primary indicators of the underlying cognitive strategy selection, the strategy questionnaire was used as a complimentary measure of cognitive strategy selection.

Spatial Activity Experience. Newcombe et al. (1983) developed the spatial activity scale to measure the spatial experience of adolescents and adults. The spatial activity survey asked participants to indicate whether they had ever participated in 81 different spatial activities from 3 to 18 years of age. Spatial activities were sex-typed as masculine (e.g. football, carpentry, rock climbing), neutral (e.g. bowling, painting, photography) and feminine (e.g. ballet, gymnastics, baton twirling). There were 40 masculine sex-typed, 21 feminine sex-typed and 20 neutral sex-typed items on the spatial activity scale. Participants indicated the degree to which they participated in each of the 81 spatial activities, on a scale ranging from ' 1 ' = 'never participated' to ' 6 ' $=$ 'participated more than once a week'. The complete spatial activity experience survey is presented in Appendix B.

The spatial activity experience survey was included to address the developmental role of early spatial activity experience in cognitive strategy selection and consequently in mental rotation performance.

\section{Dependent Variable}

Mental rotation score. The mental rotation score (MRT score) is a number ranging from 0 to 40 determined by the number of accurate responses (match or non- 
match) participants make on the Shepard and Metzler (1971) mental rotation task. This score was used to gauge a participant's mental rotation performance.

\section{Independent and Mediating Variables}

Cognitive strategy selection. Cognitive strategy selection was measured in three different ways: Eye-tracking parameters, self-report (verbal protocol) and self-rating (strategy questionnaire).

Eye-tracking parameters. Cognitive strategy was calculated as a latent variable with the different eye movement parameters as latent indicators of cognitive strategy selection. The fixation counts, fixation durations, visit durations and visit counts (see Appendix A for detailed description of each eye movement parameter) were used as latent indicators of cognitive strategy selection. Eye movement was used as the primary indicator of the underlying cognitive strategy selected.

Verbal protocol. At the end of the eye tracking study component, participants were asked a series of questions (see Appendix B). These video recordings were transcribed and coded for words that indicated different cognitive strategies (see Table 2). In response to question 1 (i.e., " How did you decide whether the two figures were a match or a non-match?'), participants who used words like 'rotation', 'flipping', 'turning in my head' etc. to describe their strategy were coded as using a holistic strategy. Participants who used words like 'counted', 'arm of figure', etc. to describe their strategy were coded as using a piecemeal strategy. Participants who used words from both categories were coded as using a mixed strategy. Thus, categorical scores ranged from 0-3 depending upon the words used to describe a strategy. In response to question 4 (i.e. "Did you use the red cube in any way while making your decision?") in the 
facilitating cue (FC) and inhibiting cue (IC) conditions, participant responses were coded as 0 or 1, depending on whether they responded with a yes or a no respectively.

Strategy Questionnaire. In the online survey, participants responded to the strategy questionnaire. Scores were cumulative for items describing the same strategy. Items 1 to 4 described a holistic strategy and scores could range from 0-28. Items 5 and 6 described a piecemeal strategy and scores could range from 0-14. A higher score in either strategy would indicate that the participant relied more on that particular strategy.

The eye-tracking parameters, verbal protocol and strategy questionnaire were used as complimentary methods in order to understand cognitive strategy selection and its role in mental rotation performance.

\section{Spatial activity experience.}

Masculine sex-typed. Of the 81 items in the spatial activity experience survey (Newcombe et al., 1983), there were 40 items sex-typed as masculine ( e.g. football, carpentry). Participants received 1 point for each masculine activity engaged in as youth. Thus, scores could range from 0 to 40 .

Feminine sex-typed. Of the 81 items in the spatial activity experience survey (Newcombe et al., 1983), there were 21 items sex-typed as feminine (e.g. ballet, gymnastics). Participants received 1 point for each feminine activity engaged in as youth. Thus, scores could range from 0 to 21 .

Neutral. Of the 81 items in the spatial activity experience survey (Newcombe et al., 1983), there were 20 items that were neither masculine nor feminine sex-typed (e.g. bowling, painting). Participants received 1 point for each neutral activity engaged in as youth. Thus, scores could range from 0 to 20 . 
The data collected in each experimental condition were analyzed on two levels (1) Statistical analysis of pre-determined eye-tracking parameters like, eye fixations, saccades, visit counts, etc. and (2) Time series analysis of eye-gaze data using change point analysis. The Tobii I-VT (velocity threshold identification) filter was used for the statistical analysis of eye-tracking parameters. The size of the area of interest (AOI) that was analyzed varied from the entire image to a single red cube, depending upon the experimental condition (see Figures $10 \& 11$ ). 


\section{PRELIMINARY ANALYSES}

The three studies included in the current dissertation draw inferences from comparisons across three experimental conditions - monochromatic, facilitating cue (FC) and inhibiting cue (IC) conditions. Although participants had been randomly assigned to one of the three experimental conditions, it was important that there be no pre-existing differences between the three participant groups. The demographics survey information was used to affirm similarity among participant groups. Tables 1 and 3 show a detailed comparison of age, GPA, ethnicity, race, spatial anxiety and general anxiety between the three experimental conditions. The three groups of participants showed no significant differences prior to the mental rotation task.

\section{Participant Exclusion Criteria}

Participants were excluded from the final analysis if one or both of the following situations occurred (1) System crashes or technical issues with the eye-tracker resulting in either no eye movement recordings or inconsistent presentation of the stimuli. (2) A task completion time of two standard deviations from the mean indicating a lack of interest/motivation, a guessing strategy or having not understood the task. On the basis of one or both of these criteria, 12 participants were excluded from the final analysis. This was approximately $3 \%$ of the total sample.

\section{Normality and Outlier Analysis}

Prior to conducting the primary analyses, descriptive statistics for all study variables were calculated (see Table 4). Data were examined for normality and outliers. With regard to normality, skewness and kurtosis were used as indicators of univariate normality, with absolute values greater than 2.3 indicating severe non-normality (Lei \& 
Lomax, 2005). Kurtosis and skewness for all variables were within acceptable ranges.

Data were also evaluated for non-model based outliers by examining leverage indices for each individual and defining an outlier as a leverage score four times greater than the mean leverage. No outliers were found.

\section{Mental Rotation Performance}

Results of a one-way ANOVA suggest that there is a significant difference in mental rotation performance across the three experimental conditions, $F(2,372)=12.72$, $p<.001$. A Post hoc analysis revealed that there was no significant difference in mental rotation performance between the monochromatic condition $(M=29.54, S D=6.52)$ and the facilitating cue (FC) condition $(M=28.84, S D=6.27), \mathrm{t}(253)=.84, p>.05, d=.1$. However, there was a significant difference in mental rotation performance between the inhibiting cue (IC) condition $(M=25.58, S D=7.33)$ and the monochromatic condition $(M=29.54, S D=6.52), t(280)=4.78, p<.001, \mathrm{~d}=.57$. There was a significant difference in mental rotation performance between the inhibiting cue (IC) $(M=25.58, S D=7.33)$ condition and the facilitating cue (FC) condition $(M=28.84, S D=6.27), t(211)=3.43, p<$ $.001, d=.48($ see Table 5)

Sex Differences in Mental Rotation Performance. In the monochromatic condition, males $(M=31.98, S D=5.72)$ significantly outperformed females $(M=28.42$, $S D=6.59), t(160)=3.32, p<.001, d=.58$.In the facilitating cue $(\mathrm{FC})$ condition, there was no significant sex differences with males $(M=30.20, S D=6.25)$ performing only slightly better than females $(M=28.47, S D=6.26), t(91)=1.10, p>.05, d=.27$. In the inhibiting cue condition, there was a significant sex difference with males $(M=28.28, S D=8.20)$ outperforming females $(M=24.59, S D=6.77), t(118)=2.49, p<.05, d=.5$ (see Table 6) 
Figure 12 shows the mental rotation performance in each experimental condition by participant sex.

Interaction between Participant Sex and Experimental Condition. There was no significant interaction effect between participant sex and experimental condition on mental rotation performance $F(2,369)=0.50, p=.61$

\section{Female Mental Rotation Performance Across Experimental Conditions.}

There was no significant difference between females in the monochromatic condition $(M=28.42, S D=6.59)$ and females in the facilitating cue $(\mathrm{FC})$ condition $(M=28.47$, $S D=6.26), t(182)=.05, p=.95, d=.0$. However, females in the inhibiting cue (IC) condition $(M=24.59, S D=6.77)$, did significantly worse than females in the monochromatic condition $(M=28.42, S D=6.59), t(197)=4.02, p<.001, d=.57$ and females in the facilitating cue (FC) condition $(M=28.47, S D=6.26), t(159)=3.75, p<.001$, $d=.6$

Male Mental Rotation Performance Across Experimental Conditions. There was no significant difference between males in the monochromatic condition $(M=32.98$, $S D=5.72)$ and males in the facilitating cue $(\mathrm{FC})$ condition $(M=30.20, S D=6.25), t(69)=$ $1.79, p>.05, d=.46$. There was no significant difference between males in the facilitating cue $(\mathrm{FC})$ condition $(M=30.20, S D=6.25)$ and the inhibiting cue (IC) condition $(M=28.28$, $S D=8.20), t(50)=.90, p>.05, d=.26$. Interestingly, though, males performed significantly worse in the inhibiting cue (IC) condition $(M=28.28, S D=8.20)$ as compared to males in the monochromatic condition $(M=32.98, S D=5.72), t(81)=3.08, p<.01, d=.6$. 


\section{Relation between Self -report Video Protocol and mental rotation performance.}

Monochromatic Condition. A one-way ANOVA revealed a significant difference in mental rotation performance as a function of reported cognitive strategies $F(2$, $53.15)=10.51, p<.001$. Since the homogeneity of variance assumption was violated, the value reported here is Welch's F. The violation of the homogeneity of variance assumption along with unequal sample sizes was addressed using the Games-Howell post hoc procedure. A Games-Howell post hoc test revealed significant differences in mental rotation performance between participants who reported selecting a holistic strategy $(M=31.14, S D=5.85)$ and participants who reported selecting a piecemeal strategy $(\mathrm{M}=25.44, S D=5.87), p<.001, d=.97$. There were no significant performance differences between the holistic $(M=31.14, S D=5.85)$ and the mixed $(M=28.13, S D=7.41), p=.10, d=$. 45 reported strategies. There were no significant performance differences between the piecemeal $(M=25.44, S D=5.87)$, and the mixed $(M=28.13, S D=7.41), \mathrm{p}=.28, d=.39$ reported strategies (see Table 7)

Facilitating Cue (FC) Condition. A one-way ANOVA revealed a significant difference in mental rotation performance as a function of reported cognitive strategies $F$ $(2,86)=5.04, p=.01$. A Hochberg's GT2 post hoc test was run to address the unequal sample sizes. The Post hoc test revealed significant differences in mental rotation performance between participants who reported selecting a holistic strategy $(M=30.19$, $S D=5.89)$ and participants who reported selecting a piecemeal strategy $(M=25.45$, $S D=6.12), p=.01, d=.79$ during the mental rotation task. There were no significant performance differences between the holistic $(M=30.19, S D=5.89)$ and the mixed ( $M=28.75, S D=6.18), p=.89, d=.24$ reported strategies. There were no significant 
performance differences between the piecemeal $(M=25.45, S D=6.12)$, and the mixed ( $M=28.75, S D=6.18), p=.46, d=.54$ reported strategies (see Table 7)

Inhibiting Cue (IC) Condition. A one-way ANOVA revealed a significant difference in mental rotation performance as a function of reported cognitive strategies $F$ $(2,49.11)=4.09, p=.023$. Since the homogeneity of variance assumption was violated, the value reported here is Welch's F. The violation of the homogeneity of variance assumption along with unequal sample sizes was addressed using the Games-Howell post hoc procedure. A Games-Howell post hoc test revealed significant differences in mental rotation performance between participants who reported selecting a holistic strategy $(M=27.05, S D=8.31)$ and participants who reported selecting a piecemeal strategy ( $M=23.00, S D=6.24), p=.03, d=.55$. There were no significant performance differences between the holistic $(M=27.05, S D=8.31)$ and the mixed $(M=26.50, S D=5.03), p=.94, d=$ .08 reported strategies. There were no significant performance differences between the piecemeal $(M=23.00, S D=6.24)$, and the mixed $(M=26.50, S D=5.03), p=.10, d=.61$ reported strategies (see Table 7)

Figures 13, 14 and 15 show the relation between cognitive strategy selection (as coded from video recordings of participant self-reports) and mental rotation performance across experimental conditions.

\section{Relation between Strategy Questionnaire and Mental Rotation Performance.}

Table 8 provides the mean estimates of each item in the strategy questionnaire for the three experimental conditions. There were significant correlations between the different items in the strategy questionnaire. Table 9 provides a summary of the correlations between the different items in the strategy questionnaire in the three 
experimental conditions. The significant positive correlations between items describing different strategies could be interpreted in three ways (1) Participants were more likely to use a combination of holistic and piecemeal strategies during mental rotation and therefore there was a high correlation between the different items (2) The strategy questionnaire was administered after the participant had answered other measures in the online survey and were more likely to have forgotten what strategies they had used during the mental rotation task and/or (2) Cognitive strategy selection is a subconscious process and may require awareness of the different strategies in order to accurately identify the strategy selected during mental rotation. In which case, a self-rating using a strategy questionnaire may not be the best way of collecting strategy information.

Monochromatic Condition. Item 2 of the strategy questionnaire "I rotated the stimulus on the right to match the stimulus on the left." was positively correlated to mental rotation performance $(r=.27, p<.001)$. Item 2 described a holistic strategy. There were no other significant correlations between any of the items on the strategy questionnaire and mental rotation performance.

Facilitating Cue (FC) Condition. There were no significant correlations between any of the items on the strategy questionnaire and mental rotation performance.

Inhibiting Cue (IC) Condition. Mental rotation performance was significantly correlated with item 2 of the strategy questionnaire "I rotated the stimulus on the right to match the stimulus on the left." $(r=.25, p=.01)$ and item 3 "I rotated the stimulus as a single whole object." $(r=.24, p=.01)$. Both items described a holistic strategy.

Figure 16 shows the relation between the proportions of holistic to piecemeal item ratings and mental rotation performance. 


\section{Discussion}

Participants were randomly assigned to one of three experimental conditions monochromatic, facilitating cue (FC) and inhibiting cue (IC) conditions respectively. The three experimental conditions differed in terms of the amount and type of non-spatial information present in the stimuli during the mental rotation task. Because of the presence of facilitating non-spatial information, participants in the FC condition were expected to score the highest of the three conditions. As a result of the presence of inhibiting non-spatial information, participants in the IC condition were expected to score the lowest of the three conditions. As predicted, participants in the IC condition performed significantly worse than the other two experimental conditions. However, participants in the FC condition did not significantly outperform participants in the monochromatic condition. The different effects of non-spatial information on mental rotation performance suggests that it is the absence of inhibiting non-spatial information, rather than the presence of facilitating non-spatial information that can significantly enhance mental rotation performance.

Consistent with spatial literature, significant sex differences, with males out performing females, were found in two out of the three experimental conditions. There were no sex differences found in the facilitating cue (FC) condition. A possible explanation is that there was not enough statistical power to detect sex differences in the facilitating cue (FC) condition and therefore the lack of sex differences should be interpreted with caution. However, given the large effect sizes of sex differences seen in mental rotation literature eliminating the need for large sample sizes, this finding could be also attributed to the fact that males scored slightly lower and females scored slightly 
higher as compared to males and females in the monochromatic condition. This could mean that although the presence of facilitating non-spatial information marginally improved performance in females, it resulted in marginally poorer performance in males. As a consequence, the overall group performance for the facilitating condition stayed the same as the monochromatic condition although the sex differences disappeared.

With regard to the presence of non-spatial information, male and female mental rotation performance was affected differently. Female mental rotation score was significantly higher in the facilitating cue condition than the inhibiting cue condition, suggesting that female mental rotators are negatively affected by the presence of distracting non-spatial cues. On the other hand, male mental rotation performance was not significantly different in the facilitating cue and inhibiting cue conditions, suggesting that male mental rotators are less affected by the presence of any type of non-spatial information. The sex differences in the relation between non-spatial information and mental rotation performance is consistent with spatial literature suggesting that good mental rotators are less likely to be affected by non-spatial information in the environment. However, the lack of difference in male performance could also be attributed to poor statistical power, given the small proportion of male participants in the facilitating cue condition. Taken together with the earlier discussion on sex differences, results suggest that it is the absence of distracting cues rather than the presence of facilitating cues that enhances female mental rotation performance. 


\section{STUDY I - COGNITIVE STRATEGY SELECTION IN MENTAL ROTATION Research Question}

Cognitive strategy refers to an individual's preferential or habitual manner of processing information for problem solving (Kozhevnikov, 2007). By definition, it would appear that mental rotation would involve visualization and transformation of mental images. Spatial literature describes several different cognitive strategies selected from during mental rotation (Hegarty, 2010; Khooshabeh et al., 2012; Kozhevnikov et al., 2002; Shepard \& Metzler, 1971; Vandenberg \& Kuse, 1978). Broadly speaking, most cognitive strategies can be grouped into two categories - holistic and piecemeal. A holistic strategy is one in which an individual mentally rotates an image as a whole and a piecemeal strategy is one in which an individual breaks down the image into several pieces for rotation and comparison (Khooshabeh et al., 2012). However, there is still much debate surrounding the definition and effectiveness of these cognitive strategies. In addition, most research on cognitive strategy relies on participant self-report for strategy information, which may be suggestive in nature. The current study was designed to address the following research questions (1) what are the different cognitive strategies selected during mental rotation? (2) Which strategy is associated with better mental rotation performance? (3) Are there sex differences in cognitive strategy selection? Pre-

defined eye-movement parameters like fixation counts, visit counts, fixation duration and visit duration were used as indirect measures of the underlying cognitive processes.

It was hypothesized that there would be two distinct eye movement patterns indicative of a holistic and piecemeal strategy respectively. A holistic strategy would be characterized by high fixation duration and low visit counts indicative of high cognitive 
load and fewer comparisons between the paired figures. A piecemeal strategy would be characterized by low fixation duration and high visit counts indicative of rapid eye movement shifts for multiple comparisons between the paired figures. Consistent with the existing spatial literature, a holistic strategy would be associated with a higher mental rotation score as compared to a piecemeal strategy. Finally, male participants would be represented in a significantly higher proportion in the more effective eye -pattern group as compared to female participants.

\section{Method}

Participants. The final sample consisted of 387 unique participants across three experimental conditions ( $M_{a g e}=20.72$ years, $S D=2.42$ years; 109 male, 278 female). Participants were randomly assigned to three experimental conditions depending on the amount and type of non-spatial information presented during the mental rotation task. There were 164 participants in the monochromatic condition (52 male, 112 female), 93 participants in the facilitating cue (FC) condition (20 male, 73 female) and 130 participants in the inhibiting cue (IC) condition (37 male, 93 female), respectively. See Tables 1 and 2 for participant demographic information.

\section{Materials.}

Hardware and Software. A standard Dell desktop with $2.8 \mathrm{GHz}$ processor and 8 GB of RAM was used to present the stimuli. The 40 stimuli per experimental condition were presented on a Sony Bravia, (model number: KDL-46EX500) flat screen TV monitor using Tobii Studio 3.2, an eye tracking analysis and visualization software compatible with Tobii X60 eye trackers. A Tobii X60 eye tracker was used to record participant gaze data. A standard Dell Desktop with $3.40 \mathrm{GHz}$ processor and $6 \mathrm{~GB}$ of 
RAM was used to analyze the Tobii recordings. A standard Dell desktop with $3.20 \mathrm{GHz}$ processor and $8 \mathrm{~GB}$ of RAM was used to run statistical analysis using MPLUS version 7.

Stimuli. Each stimulus consisted of two figures of 3-D assembled cubes used by Shepard and Metzler (1971) (see Figures $1 \& 2$ ), but had been adapted for the purposes of the current dissertation (see Figures $6 \&$ 7). In the monochromatic condition, there was no color cubes. In the facilitating cue (FC) condition, the red cube in the left figure corresponded to the red cube in the right figure. In the inhibiting cue (IC) condition the red cube in the right figure did not correspond to the red cube in the left figure

\section{Procedure.}

On consenting, participants were asked to enter the eye tracking room for the mental rotation task. After calibration, participants were shown three solved trials as well as three practice trials before the task began. Participants received feedback for their responses to the practice trials. At the end of the practice trials, participants were handed a mouse connected to the recording computer to record their decision. There were 40 trials with three rest periods of 30 seconds each after every 10 trials. The entire process took 20-25 minutes for completion.

\section{Analytic Overview}

Latent variable mixture modeling (LVMM) is a flexible analytical technique that allows participant data to be grouped into patterns of similar behavior to determine the influence of these patterns on other variables of interest. In other words, it is a way of identifying sub groups of individuals sharing similar but directly unobservable characteristics (Berlin, Williams \& Parra, 2014). Latent classes probabilistically determine participant membership i.e. each participant has a unique probability for each 
of the latent classes, for a sum total of 1 across all latent classes (Muthén \& Asparouhov, 2003). Although there are many different latent models to choose from, the current analyses used data that were cross-sectional and the latent class eye movement indicators (fixation duration, fixation count, visit duration and visit count) were continuous.

Therefore, latent profile analysis was selected as the most appropriate latent variable mixture model. One of the biggest advantages of latent profile analysis is its ability to deal with absolute magnitudes independent of sample size and the use of arbitrary score units of the latent indicators (Gibson, 1959).

Model Selection and Interpretation. The best fitting model was selected using information criteria (IC)-based fit indices - Bayesian Information Criteria (BIC; Schwartz, 1978), Akaike Information Criteria (AIC; Akaike, 1987) and Adjusted BIC (ABIC, Sclove, 1987). A lower value on the AIC, BIC and ABIC indicate a good model fit. Generally, the BIC is preferred for statistical model comparisons (Nylund, Asparouhov, \& Muthen, 2007). In addition, entropy was used to assess the accuracy with which latent models classified individual participants into their most likely latent class. Entropy values range from 0 to 1,1 representing greatest classification accuracy. Finally, the Lo-Mendell-Rubin test (LMR; Lo, Mendell, \& Rubin, 2001) and the Bootstrap Liklihood Ratio test (BLRT; McLachlan \& Peel, 2000) likelihood ratio tests were used to compare the model improvement between neighboring class models. These tests provide a $p$ value that allows the determination of statistically significant improvement in model fit for the inclusion of one more latent class. 


\section{Preliminary Analysis}

Participants were excluded from the analyses if there had been system crashes or technical issues with the eye tracker and/or if the task completion time was over two standard deviations from the mean indicating a guessing strategy or lack of understanding of task requirements. Twelve participants were excluded from the final sample, which was approximately $3 \%$ of the total sample. Prior to conducting the latent profile analysis, data were examined for normality and outliers (see chapter III for more details). Data were normal and no outliers were found.

\section{Analysis}

\section{Latent Profile Analysis Using Eye-movement Parameters as Latent Class}

Indicators of Cognitive Strategy Selection. One of the biggest advantages of latent profile analysis is that it is a model-based technique, which not only provides probability of class membership but also goodness-of-fit statistics to compare models. The eyetracking parameters of fixation duration (FD), visit duration (VD), fixation count (FC) and visit count (VC) were used as continuous latent class indicators of cognitive strategy with mental rotation score (MRT) and participant sex as latent class predictors. A total of 4 models (Class 1 to Class 4) were compared.

Monochromatic condition. The figures presented in each stimulus formed part of the area of interest (AOI) for analysis of the eye tracking parameters in the monochromatic condition (see Figure 10). Figure 17 shows a still image depicting the eye gaze pattern (path and duration) recorded by the Tobii X60 eye tracker. Eye fixation parameters of FD, VD, FC and $\mathrm{VC}$ are parsed from similar recordings using fixation filters. The results of the model fit indices are presented in Table 10. For model 2, the 
entropy value of .86 was close to 1 , signifying good precision in classification. The entropy values were almost identical for the models comprising 2, 3 and 4 latent classes. Additionally, there was a reduction in the AIC, BIC, and adjusted BIC fit indices with increasing number of latent classes indicating better model fit for the 4-class model. However, the LMR p- value indicated that the 3-class and 4-class models did not fit the data significantly better than the more parsimonious 2-class model. Hence, the 2-class latent profile model was selected. In addition, the cognitive strategy literature supports the hypothesis that there are typically 2 types of cognitive strategies selected during mental rotation i.e. holistic and piecemeal strategies.

The participants' average classification probability or posterior probabilities for the models are presented in Table 11. High certainty of classification should show high posterior probabilities in only one class but low posterior probabilities in the other classes. High posterior probabilities were observed in 2-class, 3-class and 4-class models and very minimal (near zero fractional values) overlaps between classes. The mean posterior probability for a student that belonged to a 2-class latent profile model was highest at .97. The eye-tracking parameters involving duration i.e. fixation duration (FD) and visit duration (VD) were almost identical indicating a similar mean completion time between the two latent profiles. However, latent class 1, similar to a holistic strategy had low mean fixation and visit counts (fewer switches between and within images) in comparison to latent class 2, similar to a piecemeal strategy (greater number of switches between and within images) (see Table 12).

Facilitating cue (FC) condition. The facilitating red cubes in the figures presented in each stimulus were part of the area of interest (AOI) for analyses in the 
facilitating cue condition (see Figure 11). Figure 18 shows a still image depicting the eye gaze pattern (path and duration) recorded by the Tobii X60 eye tracker. Eye fixation parameters of $\mathrm{FD}, \mathrm{VD}, \mathrm{FC}$ and $\mathrm{VC}$ are parsed from similar recordings using fixation filters. The results of the model fit indices are presented in Table 10. For model 2, the entropy value of .96 was close to 1 , signifying good precision in classification. The entropy value decreased for the 3-class and 4-class latent models respectively. There was an increase in the AIC, BIC, and adjusted BIC fit indices with increasing number of latent classes indicating better model fit for the 2-class model. Additionally, the LMR pvalue indicated that the 3-class and 4-class models did not fit the data better than the more parsimonious 2-class model. Hence, the 2-class latent profile model was selected.

The participants' average classification probability or posterior probabilities for the models are presented in Table 11. Highest posterior probabilities were observed in the 2-class latent model with very minimal (near zero fractional values) overlaps between classes. The mean posterior probability for a student that belonged to a 2-class latent profile model was highest at .99 . Similar to the monochromatic condition, the eyetracking parameters involving duration i.e., fixation duration (FD) and visit duration (VD) were almost identical indicating a similar mean completion time between the two latent profiles. However, latent class 1 had lower mean fixation and visit counts (fewer switches between and within the red cubes) suggesting a strategy in which participants did not use the facilitating non-spatial information for mental rotation. In comparison, latent class 2, had higher mean fixation and visit counts (greater number of switches between and within red cubes) suggesting a strategy in which participants used the facilitating non-spatial information for mental rotation (see Table 12). 
Inhibiting cue (IC) condition. The inhibiting red cubes in the figures presented in each stimulus were part of the area of interest (AOI) for analyses in the inhibiting cue condition (see Figure 11). Figure 19 shows a still image depicting the eye gaze pattern (path and duration) recorded by the Tobii X60 eye tracker. Eye fixation parameters of FD, VD, FC and VC are parsed from similar recordings using fixation filters. The results of the model fit indices are presented in Table 10. Although there was a reduction in the LL, AIC, BIC and ABIC values, the LMR p-value indicated no significant improvement in fit for the 2-class, 3-class and 4-class latent models in comparison to the more parsimonious 1-class latent model. It was concluded that participants did not fall into distinct cognitive strategy profiles as seen in the monochromatic and facilitating cue (FC) conditions (see Table 11).

\section{Latent Profiles as Predictors of Mental Rotation Performance and}

Participant Sex. The two latent profiles that emerged in the monochromatic and facilitating cue $(\mathrm{FC})$ conditions were used as categorical predictors of mental rotation performance and participant sex using logistic regression (see Table 13). Figures 20 and 21 show the relation between the latent classes of cognitive strategy selection and mental rotation performance in the monochromatic and facilitating cue (FC) conditions respectively.

Monochromatic condition. There was a significant difference in mental rotation performance between latent class $1(M=27.43, S D=6.31)$ and latent class $2(M=34.72$, $S D=3.44), t(147.29)=9.44, p<.001 ; d=1.43$ (see Figure $Z$ ). However, there was no significant association between the latent classes and participant $\operatorname{sex} \chi^{2}(1)=1.43, p=.23$ (see Table 14) 
Facilitating cue (FC) condition. There was a significant difference in mental rotation performance between latent class $1(M=27.84, S D=6.19)$ and latent class 2 $(M=33.29, S D=4.52), t(91)=3.43, p=.001 ; d=1.01$ (see Figure Z). A Fisher's exact test (FET) showed no significant association between the two latent classes and participant $\operatorname{sex}(p=.76)($ see Table14)

Figures 22 and 23 show the relation between the latent classes of cognitive strategy selection and mental rotation performance by participant sex in the monochromatic and facilitating cue (FC) conditions respectively.

\section{Discussion}

The current study was designed to address the following research questions (1) what are the different cognitive strategies selected during mental rotation? (2) Which strategy is associated with better mental rotation performance? (3) Are there sex differences in cognitive strategy selection? As hypothesized, a latent profile analysis revealed two distinct eye movement patterns in both the monochromatic and the facilitating cue (FC) conditions. Interestingly, no latent classes emerged in the inhibiting cue condition. It is important to note here, that the inhibiting cue condition was also the lowest performing group, even though there were no significant differences in performance between the facilitating cue (FC) and the monochromatic conditions. The difference in cognitive strategy selection suggests that (1) a well-defined cognitive strategy is associated with better mental rotation performance and (2) cognitive strategy selection is flexible and changes with the nature of spatial and non-spatial information.

Monochromatic Condition. Latent class 1 had significantly smaller fixation and visit counts than latent class 2 , an eye pattern consistent with the definition of a holistic 
strategy in which an individual rotates an image as a whole (i.e. fewer fixations on individual arms) and therefore makes fewer comparisons between the paired figures (i.e. fewer visits to each figure). Latent class 2 had significantly higher fixation and visit counts than latent class 1 , an eye pattern consistent with the definition of a piecemeal strategy in which an individual breaks down each figure into its constituent arms (i.e. higher fixations on individual arms) and makes several comparisons of these arms or pieces between the paired figures (i.e., more visits to each figure).

Spatial literature is biased towards a holistic strategy as the more effective strategy for mental rotation. In addition, preliminary analysis on participant self-reports collected during the video protocol and the online strategy questionnaire suggested that a holistic strategy was significantly associated with better mental rotation performance. However, latent class 1 , similar to that of a holistic strategy was found to be negatively associated with mental rotation performance and latent class 2 , similar to that of a piecemeal strategy was found to be positively associated with mental rotation performance. This result suggests that a piecemeal strategy and not a holistic strategy may be associated with good mental rotation performance and is task dependent, which has important implications for spatial training pedagogy and should be investigated further.

Facilitating Cue (FC) Condition. Unlike that in the monochromatic condition where the entire figure was part of the area of interest (AOI), only the facilitating red cubes in each figure was part of the AOI used for analysis in the facilitating cue (FC) condition (see Figure 11). Latent class 1 had significantly smaller fixation and visit counts than latent class 2 . This suggests that participants who fit the latent class 1 profile 
were those who fixated on and visited the red cube significantly less than participants who fit the latent class 2 profile. Since the red cube was providing facilitative non-spatial information, participants who used this cube as part of their cognitive strategy should have significantly outperformed participants who failed to use the facilitating cue. Results supported this hypothesis with latent class 2 being positively associated with mental rotation performance. The superiority of latent class 2 eye-pattern supports the argument for the use of non-spatial cues in education pedagogy that requires mental rotation skills.

In light of the fact that participants in the facilitating cue (FC) condition did not significantly outperform participants in the monochromatic condition despite the presence of a facilitating non-spatial cue, suggests that flexibility in cognitive strategy selection and not a specific cognitive strategy facilitates mental rotation performance. The concept of strategy flexibility will be built on in more detail in study 3 in the current dissertation.

Inhibiting Cue (IC) Condition. Interestingly, there were no latent classes of eye gaze parameters found in the inhibiting cue (IC) condition, the worst performing experimental condition. The lack of a well-defined strategy supports the argument that it is flexibility in cognitive strategies needed in different situations rather than one optimum strategy that facilitates mental rotation. This finding has important implications for training techniques focused on improving spatial ability.

Finally, there were no significant sex differences in cognitive strategy selection in either the monochromatic or the facilitating cue (FC) conditions, although the percentage of male participants in the more effective latent class of cognitive strategy was higher than that of female participants in both experimental conditions. Given that the 
proportion of male participants in all three experimental conditions was considerably lower than that of female participants, the statistical analysis of the relation between participant sex and cognitive strategy selection may have lacked sufficient statistical power to detect sex differences. 


\section{STUDY II - ROLE OF EARLY SPATIAL ACTIVITY EXPERIENCE IN MENTAL ROTATION PERFORMANCE}

\section{Research Question}

Sex differences seen in spatial research, specifically mental rotation (Linn \& Peterson, 1985; Maccoby \& Jacklin, 1974; D. Voyer, et al., 1995) have been attributed to biological/hormonal factors (Gaulin \& Hoffman, 1988; Hausmann, Slabbekoorn, Van Goozen, Cohen-Kettenis \& Güntürkün, 2000; Silverman \& Kastuk, Choi \& Phillips, 1999), different attentional/cognitive strategies (Hugdahl, Thomsen \& Ersland, 2006; Linn \& Peterson, 1985) as well as social/experiential factors (Baenninger \& Newcombe, 1989; 1995; Nazareth et al., 2013) among others. Research investigating social/experiential factors suggests that differential involvement in spatial activity experiences may contribute to differences in spatial performance ((Nazareth et al., 2013; Neidhardt \& Popp, 2010; Newcombe et al., 1983; Sherman, 1967; 1974). Study I examined the cognitive factor of cognitive strategy selection as a possible explanation for sex differences. The present study investigates the social/experiential factor of early spatial activity experiences, specifically those that have been sex-typed into maleappropriate (e.g., mechanical drawing, building models, carpentry) and femaleappropriate (e.g., ballet, sketching) and their effect on mental rotation performance. The current study was designed to investigate the following research questions (1) Do early spatial activity experiences predict mental rotation performance? (2) Are there specific types of spatial activities, either male sex-typed, female sex-typed or neutral activities associated with higher mental rotation performance? (3) Do early spatial activity experiences mediate sex differences observed in mental rotation performance? 


\section{Method}

Participants. A subset of the original sample population was asked to fill out the spatial activity experience survey by Newcombe et al. (1983). The final sample consisted of 191 (40 male, 151 female) unique participants across three experimental conditions Participants were randomly assigned to one of three experimental conditions depending on the amount and type of non-spatial information presented during the mental rotation task. There were 74 participants in the monochromatic condition (18 male, 56 female), 51 participants in the facilitating cue (FC) condition (6 male, 45 female) and 66 participants in the inhibiting cue (IC) condition (16 male, 50 female) respectively. Descriptive statistics for the subset of participants are presented in Tables 15 and 16. The mean and standard deviations for all study variables were not significantly different from the larger sample population.

\section{Materials.}

Hardware and Software. A standard Dell desktop with $2.8 \mathrm{GHz}$ processor and 8 GB of RAM was used to present the stimuli. The 40 stimuli per experimental condition were presented on a Sony Bravia, (model number: KDL-46EX500) flat screen TV monitor using Tobii Studio 3.2, an eye tracking analysis and visualization software compatible with Tobii X60 eye trackers. A Tobii X60 eye tracker was used to record participant gaze data. A standard Dell Desktop with $3.40 \mathrm{GHz}$ processor and $6 \mathrm{~GB}$ of RAM was used to analyze the Tobii recordings

Stimuli. Each stimulus consisted of two figures of 3-D assembled cubes used by Shepard and Metzler (1971) (see Figures $1 \& 2$ ), but had been adapted for the purposes of the current dissertation (see Figures $6 \& 7$ ). In the monochromatic condition, there were 
no color cubes. In the facilitating cue (FC) condition, the red cube in the left figure corresponded to the red cube in the right figure. In the inhibiting cue (IC) condition the red cube in the right figure did not correspond to the red cube in the left figure

\section{Measures}

Spatial Activity Experience Survey. Newcombe et al. (1983) developed the spatial activity scale to measure the spatial experience of adolescents and adults. The spatial activity survey asked participants to indicate whether they had ever participated in 81 different spatial activities from 3 to 18 years of age. Spatial activities were sex-typed as masculine (e.g., football, carpentry, rock climbing), neutral (e.g., bowling, painting, photography) and feminine (e.g., ballet, gymnastics, baton twirling). There were 40 masculine sex-typed, 21 feminine sex-typed and 20 neutral sex-typed items on the spatial activity scale. Participants received 1 point for each spatial activity engaged in as youth. Thus number of spatial activities could range from 0-81, male sex-typed from 0-40, female sex-typed from 0-21 and neutral from 0-20.

\section{Procedure}

On consenting, participants were asked to enter the eye tracking room for the mental rotation task. After calibration, participants were shown three solved trials as well as three practice trials before the task began. Participants received feedback for their responses to the practice trials. At the end of the practice trials, participants were handed a mouse connected to the recording computer to record their decision. There were 40 trials with three rest periods of 30 seconds each after every 10 trials. The entire process took 20-25 minutes for completion. Participants then responded to questions in the verbal 
protocol before completing the online survey, which included the spatial activity experience measure.

\section{Preliminary Analysis}

Participants were excluded from the analyses if there had been system crashes or technical issues with the eye tracker and/or if the task completion time was over two standard deviations from the mean indicating a guessing strategy or lack of understanding of task requirements. Seven participants were excluded from the final sample, which was less than $4 \%$ of the total sample. Prior to conducting the mediation analysis, data were examined for normality and outliers (see chapter III for more details) Data were normal and no outliers were found.

Monochromatic Condition. Consistent with earlier findings, males $(M=32.18$, $S D=5.96)$ significantly outperformed females $(M=27.70, S D=6.63)$ on the MRT, $t$ $(65)=2.47, p<.05, d=0.71$. Males $(M=12.71, S D=6.49)$ participated in significantly more masculine/male spatial activities than females $(M=8.66, S D=5.09), t(65)=2.64, p<.05$, $d=0.69$ (see Figure 24). There was no significant difference in male $(M=7.61, S D=4.35)$ and female $(M=7.71, S D=4.05)$ engagement in female sex-typed spatial activities $t(65)=$. 09, $p=.93$ (see Figure 25).

Facilitating Cue (FC) Condition. There were no sex differences in mental rotation score with male participants $(M=27.83, S D=5.19)$ performing only slightly better than female participants $(M=27.62, S D=6.46), t(49)=-0.08, p=.94$. There was a significant difference in male $(M=15.50, S D=4.04)$ and female $(M=10.38, S D=4.65)$ engagement in male sex-typed spatial activities, $t(49)=-2.57, p<.001, d=1.18$ (see Figure 24). There was no significant difference in male $(M=10.50, S D=.25)$ and female 
$(M=8.98, S D=3.20)$ engagement in female sex-typed spatial activities $t(49)=1.09, p=.28$ (see Figure 25)

Inhibiting Cue (IC) Condition. Similar to the facilitating cue (FC) condition there were no sex differences in mental rotation score with males $(M=29.63, S D=6.94)$ scoring only slightly higher than females $(M=25.78, S D=6.74), t(64)=-1.97, p=.05, d=$. 57. There was a significant difference in male $(M=13.69, S D=5.59)$ and female $(M=9.67$, $S D=5.57$ ) engagement in male sex-typed spatial activities, $t(65)=-2.51, p=.01$ (see

Figure 24). There was a significant difference in male $(M=7.31, S D=.57)$ and female $(M=9.27, S D=4.30)$ engagement in female sex-typed spatial activities $t(65)=1.72, p=.03$ (see Figure 25).

\section{Analytic Overview}

Mediation Analysis is designed to investigate the mechanism by which a predictor variable influences the outcome. The Baron and Kenny (1986) approach is one of the most widely used methods to test for mediation. However, the approach has been widely criticized for several reasons, not the least of which is low statistical power to detect mediation. The Sobel test overcomes some of the drawbacks of the Baron and Kenny (1986) method. However, a major flaw of the Sobel test is the assumption that the sampling distribution of the indirect effect is normal which is almost always violated. Hayes (2009) reviews the different methods of mediation analysis and discusses their advantages and disadvantages. Bootstrapping is an alternative to the previous two methods, providing the highest power and Type I error control (MacKinnon et al., 2004) Bootstrapping does not make any of the assumptions made by the other mediation 
methods. In the current analysis, bootstrapping with 1000 iterations was used for the mediation analysis using PROCESS, a modeling tool for SPSS (Hayes, 2012).

\section{Analysis}

\section{Relation between Early Spatial Activity Experience and Mental Rotation}

\section{Performance.}

\section{Mediation Analysis Using Early Spatial Activity Experience As a Mediator.}

Monochromatic Condition. All three variables of interest, sex of participant, number of masculine spatial activities, and MRT scores, were significantly and positively correlated with each other (Figure 26), meeting the prerequisite for a mediation analysis $(r$ 's $>0.29, p$ 's $<.05)$ Regression analysis established that sex of participant significantly predicted their MRT scores $(\beta=.29, t=2.47, p<.05)$. Figure 27 shows the strength of this direct effect (path $c$ ) of sex of the participant on MRT scores. When the number of masculine spatial activities was included as a potential mediator, the path coefficient $\left(c^{\prime}\right)$ reduced and was no longer significant $(\beta=.21, t=1.77, p=0.08)$ suggesting that sex of participant influences MRT scores via number of masculine spatial activities (see Figure 27). The preliminary conditions for a mediation analysis were not met by female or neutral spatial activities. The model accounted for $14.02 \%$ of the variance (using $R^{2}$ ) on $M R T$ scores and provides statistical support that the reduction in the direct effect of sex of participant on MRT scores was significant, and indicates a partial mediation of $M R T$ score.

Facilitating Cue (FC) Condition. Since there were no sex differences a mediation analysis could not be run. There were no significant relations between mental rotation 
performance and early male sex-typed $(r=.06, p=.68)$ or female sex-typed $(r=-.23, p=$. 11) spatial activities.

Inhibiting Cue (IC) Condition. Since there were no sex differences, a mediation analysis could not be run. There were no significant relations between mental rotation performance and early male sex-typed $(r=.002, p=.99)$ or female sex-typed $(r=-.14, p=$. 25) spatial activities.

\section{Discussion}

The present study investigates the role of early spatial activity experience, particularly those spatial activities that have been sex-typed as masculine/male-oriented in mental rotation performance. In the monochromatic condition, results suggest that the relation between sex of the participant and MRT score is partially mediated and explained by the number of masculine spatial activities participants had engaged in as youth.

Female sex-typed and neutral spatial activities did not demonstrate a similar mediating effect on sex differences in mental rotation performance. The mediating effect of early male sex-typed spatial activity experience supports the argument that spatial skill is malleable (Uttal et al., 2013). Doyle et al. (2012) discuss the differential exposure in favor of boys, to male sex-typed spatial content like contact sports, toys requiring manipulation in space and mathematics and science. Similarly, Baenninger and Newcombe (1995) discuss the formal and informal environmental experiences facilitating spatial ability that are differentially available to boys and girls. Encouraging female youth to engage in particular types of spatial activity experiences could help bridge the gap in spatial ability between the sexes. Spatial ability relates to future Science, Technology, Engineering and Mathematics (STEM) success (Shea, Lubinski \& Benbow, 2001; Wai et 
al., 2009) and addressing the factors that contribute to sex differences in spatial ability is of paramount importance.

However, the mediating effect of male sex-typed spatial activities was only seen in the monochromatic condition. Since there were no sex differences in the facilitating cue (FC) and the inhibiting cue conditions, the mediating relation of male sex-typed spatial activities could not be examined. The lack of sex differences could be attributed to the small proportions of males in the non-spatial cue conditions i.e. the facilitating cue and inhibiting cue conditions and consequently low statistical power. However, what is interesting in these two non-spatial cue conditions is that there was no significant correlation between mental rotation performance and early spatial activity experience, either male sex-typed or female sex-typed. The overarching research question guiding this dissertation was: Do experiential factors affect adult mental rotation performance through the development of an optimum cognitive strategy? However, results from this study indicate that experiential factors may predict mental rotation performance in the absence of any non-spatial information i.e. with no additional non-spatial complexities in the stimuli. This finding points to a more complex relation between early spatial activity experience and mental rotation performance than initially predicted. One hypothesis is that experiential factors may be limited to influencing the development of a cognitive strategy without influencing the flexibility of strategy selection needed for different types of stimuli. Study 3 builds on this research question by exploring the relation between cognitive strategy selection, strategy flexibility and early spatial activity experience. 
VII. STUDY III - RELATION BETWEEN COGNITIVE STRATEGY SELECTION, STRATEGY FLEXIBILITY AND EARLY SPATIAL ACTIVITY EXPERIENCE

\section{Research Question}

This study investigates the overarching developmental question that guides the current dissertation - Do experiential factors affect adult mental rotation performance through the development of an optimum cognitive strategy? The preceding studies, study I and study II examined the cognitive and social/experiential factors that affect adult mental rotation performance independent of each other. The goal of this study is to examine the relation between the cognitive factor of strategy selection and the experiential factor of early spatial activity experience. Thus, the results of study I and II act as preliminary analysis for study III.

Results of study I suggest that there are two distinct cognitive strategies, indicated by the 2-class latent model composed of eye-movement indicators. The two distinct cognitive strategies were significant predictors of mental rotation performance in the monochromatic and the facilitating cue (FC) conditions. No distinct latent classes emerged in the inhibiting cue (IC) condition, which performed significantly worse than the monochromatic and facilitating cue (FC) conditions. Study I identified the different cognitive strategies and also confirmed the importance of cognitive strategy selection for

mental rotation performance, with one strategy being better than the other. For the sake of convenience, study III was divided into two parts. In the first part of study III, it was hypothesized that the latent classes indicative of the different cognitive strategies would significantly predict early spatial activity experience in the monochromatic and facilitating cue (FC) conditions respectively. 
Results of study II suggested a complex relation between early spatial activity experience and mental rotation performance. One plausible hypothesis is that the experiential factor of early spatial activity experience might be limited to influencing the development of a cognitive strategy without influencing the flexibility of strategy selection needed for different types of stimuli. The second part of study III built on this research question by exploring the relation between strategy flexibility and early spatial activity experience.

\section{Relation between cognitive strategy selection and early spatial activity experience}

\section{Part 1. Method}

Participants. The subset of participants analyzed in study II was used for the first part of study III. The final sample consisted of 191 ( 40 male, 151 female) unique participants across three experimental conditions (see Tables $15 \& 16$ for a complete list of participant demographics)

\section{Materials}

Hardware and Software. A standard Dell desktop with $2.8 \mathrm{GHz}$ processor and 8 GB of RAM was used to present the stimuli. The 40 stimuli per experimental condition were presented on a Sony Bravia, (model number: KDL-46EX500) flat screen TV monitor using Tobii Studio 3.2, an eye tracking analysis and visualization software compatible with Tobii X60 eye trackers. A Tobii X60 eye tracker was used to record participant gaze data. A standard Dell Desktop with $3.40 \mathrm{GHz}$ processor and $6 \mathrm{~GB}$ of RAM was used to analyze the Tobii recordings. The statistical software $\mathrm{R}$ was used to analyze the data. 
Stimuli. Each stimulus consisted of two figures of 3-D assembled cubes used by Shepard and Metzler (1971) (see Figures $1 \& 2$ ), but had been adapted for the purposes of the current dissertation (see Figures $6 \& 7$ ). In the monochromatic condition, there were no color cubes. In the facilitating cue (FC) condition, the red cube in the left figure corresponded to the red cube in the right figure. In the inhibiting cue (IC) condition the red cube in the right figure did not correspond to the red cube in the left figure

\section{Procedure}

On consenting, participants were asked to enter the eye tracking room for the mental rotation task. After calibration, participants were shown three solved trials as well as three practice trials before the task began. Participants received feedback for their responses to the practice trials. At the end of the practice trials, participants were handed a mouse connected to the recording computer to record their decision. There were 40 trials with three rest periods of 30 seconds each after every 10 trials. The entire process took 20-25 minutes for completion.

\section{Analytic Overview}

Latent variable mixture modeling (LVMM) is a flexible analytical technique that allows participant data to be grouped into patterns of similar behavior to determine the influence of these patterns on other variables of interest. In other words, it is a way of identifying sub groups of individuals sharing similar but directly unobservable characteristics (Berlin, Williams \& Parra, 2014). Latent classes probabilistically determine participant membership i.e. each participant has a unique probability for each of the latent classes, for a sum total of 1 across all latent classes (Muthén \& Asparouhov, 2003). Although there are many different latent models to choose from, the current 
analyses used data that were cross-sectional and the latent class eye movement indicators (fixation duration, fixation count, visit duration and visit count) were continuous.

Therefore, latent profile analysis was selected as the most appropriate latent variable mixture model.

\section{Preliminary Analysis}

Participants were excluded from the analyses if there had been system crashes or technical issues with the eye tracker and/or if the task completion time was over two standard deviations from the mean indicating a guessing strategy or lack of understanding of task requirements. Prior to conducting the latent profile analysis, data were examined for normality and outliers (see chapter III for more details) Data were normal and no outliers were found.

\section{Analysis}

Latent Profiles as Predictors of Early Spatial Activity Experience. The eyetracking parameters of fixation duration (FD), visit duration (VD), fixation count (FC) and visit count (VC) were used as continuous latent class indicators of cognitive strategy (see study I for full analysis). The two latent profiles that emerged in the monochromatic and facilitating cue (FC) conditions were used as categorical predictors of male sextyped, female sex-typed and neutral early spatial activity experiences.

Monochromatic condition. The latent classes of cognitive strategy selection significantly predicted male sex-typed spatial activity experience, $t(77)=2.32, p<.05$, C.I. $=.41$ to $.03 d=.6$ (see Figure 28). The latent classes of cognitive strategy selection did not significantly predict female sex-typed spatial activity experience $t(77)=1.93$, $p>.05$. 
Facilitating cue (FC) condition. The latent classes of cognitive strategy selection did not significantly predict male sex-typed spatial activity experience, $t(49)=1.58, p>$ .05 (see Figure 28) or female sex-typed spatial activity experience, $t(49)=.87, p>.05$

Inhibiting Cue (IC) Condition. Latent profile analysis suggested a one class latent model and hence the relation between the latent variable of cognitive strategy selection and mental rotation performance could not be examined for this experimental condition.

\section{Discussion}

The first part of study III investigated the developmental role of early spatial activity experience in the selection of an optimum cognitive strategy.

Monochromatic Condition. As hypothesized, greater participation in spatial activities was significantly associated with the latent class predictive of a higher mental rotation score in the monochromatic condition. This supports the argument that early engagement with spatial content aids in the development of an optimum cognitive strategy. Certain types of spatial activities specifically male sex-typed activities were found to be significantly associated with cognitive strategy in the monochromatic condition. It is important to note that female sex-typed spatial activities were not found to be significantly associated with cognitive strategy selection. This result is consistent with the findings of study II, where female sex-typed spatial activity experience did not mediate the relation between participant sex and mental rotation performance.

Facilitating Cue (FC) Condition. Contrary to the hypothesis, there was no significant relation between early spatial activity experience and cognitive strategy selection. It is important to note here, that the latent classes for the FC experimental 
condition do not indicate a holistic and piecemeal strategy. Rather, they indicate the flexibility of traditional cognitive strategies to take into account facilitating non-spatial information. Since the area of interest (AOI) was restricted to the red cube, the latent classes represented the extent to which a participant could incorporate facilitating cues to improve overall accuracy and speed. Therefore, the lack of a significant relation does not imply that early spatial activity experience does not play a developmental role in cognitive strategy selection. It may however suggest, that spatial activity experience may not play a developmental role in strategy flexibility. Interestingly, there had been no significant relation between spatial activity experience and mental rotation performance for the FC experimental condition. This further corroborates the hypothesis that spatial activity experience may not influence strategy flexibility needed in the case of modified stimuli.

\section{The Role of strategy flexibility in Mental Rotation Performance}

\section{Part 2. Method}

Participants. The total participant pool was used for the change point analysis. The final sample consisted of 387 unique participants across three experimental conditions $\left(M_{a g e}=20.72\right.$ years, $S D=2.42$ years; 109 male, 278 female) (see Table 1 for a complete list of participant demographic information).

\section{Materials.}

Hardware and Software. A standard Dell desktop with $2.8 \mathrm{GHz}$ processor and 8 GB of RAM was used to present the stimuli. The 40 stimuli per experimental condition were presented on a Sony Bravia, (model number: KDL-46EX500) flat screen TV monitor using Tobii Studio 3.2, an eye tracking analysis and visualization software 
compatible with Tobii X60 eye trackers. A Tobii X60 eye tracker was used to record participant gaze data. A standard Dell Desktop with $3.40 \mathrm{GHz}$ processor and $6 \mathrm{~GB}$ of RAM was used to analyze the Tobii recordings. The Statistical tool, $R$ was used to run the change point analysis.

Stimuli Description. Each stimulus consisted of two figures of 3-D assembled cubes used by Shepard and Metzler (1971) (see Figures $1 \& 2$ ). The stimuli contained a non-spatial color cue in the facilitating cue (FC) and inhibiting cue (IC) conditions. (see Figures $6 \& 7$ )

\section{Procedure}

On consenting, participants were asked to enter the eye tracking room for the mental rotation task. There were 40 trials with three rest periods of 30 seconds each after every 10 trials. The entire process took 20-25 minutes for completion.

\section{Analytic Overview}

In order to investigate strategy flexibility, it was necessary to deviate from traditional analysis examining pre-defined eye tracking parameters of fixations and saccades and to analyze raw time-series data of eye positions provided by the eye tracker. Raw eye movement time series analysis helps to overcome the limitations imposed by fixation filters in different eye trackers. It also facilitates the investigation of changes in eye movement pattern from one stimulus to the next.

Thus, in order to study strategy flexibility, a within-subject statistical method was needed that could analyze changes in eye gaze patterns from among thousands of eye positions per participant for 387 participants across experimental conditions. Each participant, irrespective of the different experimental conditions, had been presented with 
40 stimuli in addition to the calibration and sample stimuli. The eye tracker provided eye position information every 16 milliseconds. Therefore, the statistical method would have to be suitable to identify the location of multiple change points (of the order of 100) to detect changes in eye patterns between stimuli and within stimuli.

Changepoint analysis is used to identify changes in statistical properties within a given time series (Killick, Fearnhead \& Eckley, 2012). Three commonly used changepoint algorithms are the Binary segmentation (BinSeg), Pruned Exact Linear Time (PELT) and Segment Neighborhoods (SegNeigh) methods respectively. The PELT algorithm is more computational efficient and has increased accuracy in multiple changepoint detection (Killick et al., 2012). The changepoint analysis used for the current dissertation used the PELT algorithm for multiple changepoint detection. The mathematical equation defining the PELT algorithm is beyond the scope of the current dissertation and can be found in great detail in Killick et al., (2012).

\section{Preliminary analysis}

Raw eye gaze time series data comprising information regarding the x-position of participants eye gaze at any given time point was used for the change point analysis. Eye movement during the calibration process at the beginning and at the end of the mental rotation task was removed from the within-subject analysis. In addition, eye movement data recorded during the trial and practice sessions at the beginning of the mental rotation task was removed from the analysis. Each participant's cleaned eye gaze time series for the 40 stimuli was then run through the PELT algorithm in R, and the number of change points detected was used as an indicator of strategy flexibility and its relation to spatial activity experience and mental rotation performance (see Figure 29). Participant eye gaze 
data were excluded from the change point analysis if the eye tracker had recorded less than $50 \%$ of participant eye movement. Table 17 presents the final sample size after applying the exclusion criterion in each of the three experimental conditions.

\section{Analysis}

Relation between cognitive strategy flexibility and mental rotation performance

In each of the three experimental conditions, there was a strong positive correlation between the number of change points detected by the PELT algorithm and mental rotation performance ( $\mathrm{rs}>.45$ ), controlling for spatial anxiety (see Table 18). Figures 30,31 and 32 present scatterplots of the relation between number of changepoints detected and mental rotation performance in the monochromatic, facilitating cue (FC) and inhibiting cue (IC) conditions respectively. There were no significant differences in the number of change points detected for participants in the monochromatic condition ( $M=$ 381.97, $S D=200.99)$, facilitating cue (FC) condition $(M=392.66, S D=180.32)$, and inhibiting cue (IC) condition $(M=409.28, S D=233.33)$, Welch's $F(2,191.12)=.43, p=$ .65. In addition, there was no sex difference in the number of change points detected in the three experimental conditions. The means and standard deviations for the number of change points detected for male and female participants in the three experimental conditions are presented in Table 19.

Relation between cognitive strategy flexibility and early spatial activity experience

In the monochromatic condition, there was a significant positive correlation between participation in early spatial activities and number of changepoints detected ( $r=$. $31, p=.008)$. Table 20 presents correlations for male sex-typed, female sex-typed and neutral spatial activity experience. However, there were no significant correlations 
between participation in early spatial activities and number of changepoints detected in the facilitating cue $(r=-.06, p=.71)$ and the inhibiting cue $(r=.10, p=.46)$ conditions respectively.

\section{Discussion}

The second part of study III investigated the complex relation between early spatial activity experience and cognitive strategy flexibility. It was hypothesized that experiential factors may be limited to influencing the development of a cognitive strategy without influencing the flexibility of strategy selection needed for different types of stimuli, for example, complex stimuli comprising non-spatial information. Results support this hypothesis. In all three experimental conditions, cognitive strategy flexibility was positively correlated to mental rotation performance. This suggests that switching between cognitive strategies depending on task complexity enhances mental rotation performance. This contradicts the existing assumption that there is one optimum cognitive strategy for mental rotation and that strategy is similar to a holistic approach to mental rotation. Instead, the current finding supports the view that there is one optimum strategy for every type of stimuli and the flexibility to switch between strategies based on stimuli type is what separates the good performers from the poor performers. In other words, participants who use a holistic strategy may not necessarily be better than participants who use a piecemeal strategy or vice versa. Participants who are capable of rapidly switching between holistic and piecemeal strategies given certain spatial or nonspatial information tend to outperform participants who rigidly choose one or the other cognitive strategy. 
The current analysis investigated the developmental role of early spatial activity experience in cognitive strategy flexibility. The non-significant relation between spatial experience and strategy flexibility in the non-spatial cue conditions (i.e. facilitating cue and inhibiting cue conditions) suggest that early spatial experience may influence the development of cognitive strategies but may not play as important a role in the development of strategy flexibility. The significant relation between spatial experience and strategy flexibility in the monochromatic condition can be attributed to the fact that participants were not required to manipulate non-spatial information as compared to the non-spatial cue conditions. The lack of facilitating or inhibiting cues in the monochromatic condition may have made it possible to perform well even if participants stuck to one optimum cognitive strategy throughout the mental rotation task.

Finally, results indicating non-significant sex differences in cognitive strategy flexibility should be interpreted with caution because of the low statistical power available to detect sex differences. However, the lower number of changepoints detected for female participants as compared to male participants in all three experimental conditions indicates a possible trend and should be investigated in future studies. 


\section{GENERAL DISCUSSION}

\section{Summary}

The current dissertation was designed to examine cognitive and experiential factors that affect adult mental rotation. The overarching research question guiding the dissertation was: Do experiential factors affect adult mental rotation performance through the development of an optimum cognitive strategy? On the one hand, research supports the malleability of spatial skills and the enhancing effect of experiential factors

like early spatial activity experience, on the other hand, we are still fairly uncertain about the mechanism by which experiential factors enhance mental rotation performance. The current dissertation sought to address this gap in the literature by examining cognitive strategy selection as a possible mechanism by which experiential factors like early spatial activity experience enhance mental rotation performance.

Each of the three studies included in the dissertation aimed to address a specific aspect of cognitive strategy selection and its relation to early spatial activity experience and mental rotation performance. Three experimental conditions were designed to act as groups of comparison within each study. The three experimental conditions differed in the amount and type of non-spatial information present in the stimulus. All three experimental conditions used variations of the Shepard and Metzler (1971) mental rotation task consisting of pairs of figures of 3D assembled cubes. The paired figures were either similar but rotated at different angles or mirror images of each other. In the monochromatic condition, there was no non-spatial information. In the facilitating cue (FC) condition, a red colored cube in each figure acted as a facilitating non-spatial cue. In 
the inhibiting cue (IC) condition, a red colored cube in each figure acted as an inhibiting non-spatial cue.

\section{Factors Affecting Adult Mental Rotation Performance}

\section{Amount and type of Non-spatial Information}

Results suggest that the presence of distracting non-spatial information like color negatively impacts male and female mental rotation performance. However, the presence of facilitating non-spatial information does not significantly enhance male or female mental rotation performance. This is an important finding with regard to training techniques and educational pedagogy designs aimed at enhancing mental rotation performance. Spatial training and educational material should focus on reducing the amount of distracting non-spatial information presented to the learner rather than solely on increasing the amount of facilitating non-spatial information. There was no difference in performance between male participants in the inhibiting cue and facilitating cue conditions, suggesting that the type of non-spatial cues does not affect male as much as it does female mental rotation performance. However, this can be attributed to low statistical power on account of a much smaller male participant pool and should be interpreted with caution.

\section{Cognitive Strategy Selection}

Previous research has mainly relied on self-reports, either verbal descriptions or survey checklists to understand cognitive strategy selection. However, the process of choosing a strategy while mentally rotating may not be a conscious process and therefore cannot be accessed via participant self-reports. In the current dissertation, even immediately after the task, many participants found it difficult to put into words the 
process they had used to make their decision during the mental rotation task. As expected, coded verbal reports suggested that a holistic strategy was associated with higher mental rotation performance, which is consistent with other self-report studies investigating cognitive strategy selection.

Similarly, strategy checklists may be suggestive in nature. This can be seen by the fact that most of the items in the strategy checklist used in the current dissertation were positively correlated with each other indicating that participants were not able to see that "rotating the stimulus piece by piece" and "rotating the stimulus as a single whole" were contradictory items and could not both have been true. Either the differences in the strategy items were not discernible to the participants in the current sample or their responses in fact were indicative of strategy flexibility, which would explain the high ratings for contradictory strategy items. With either assumption, the use of a strategy questionnaire alone did not help in identifying a single optimum cognitive strategy or the flexibility of strategy selection. Study I used an eye tracker to record participant eye gaze during mental rotation as an indicator of the underlying subconscious cognitive process.

Results of study I revealed two distinct eye movement patterns, based on eye fixations and saccades as indicators of the underlying cognitive strategy. In the monochromatic condition, the eye pattern resembling a piecemeal strategy was predictive of higher mental rotation performance contradicting existing research suggesting that holistic strategy is predictive of a better mental rotation performance. This finding highlights the gaps in our understanding of different cognitive strategies and their role in mental rotation performance. In the facilitating cue (FC) condition, the eye pattern incorporating the facilitating non-spatial cue in the cognitive strategy was predictive of 
better mental rotation performance. This finding contradicts the assumption of the existence of a single optimum cognitive strategy for all types of stimuli and highlights the need for flexibility in strategy selection based on the amount and type of spatial and nonspatial cues present in the stimulus. In the inhibiting cue condition, the lack of distinct eye patterns and the overall low performance in the IC group confirms the importance of cognitive strategy selection and flexibility for better mental rotation performance.

\section{Early Spatial Activity Experience}

Study II examined the role of early spatial activity experience in mental rotation performance. Male sex-typed spatial activities were found to significantly mediate the relation between participant sex and mental rotation performance in the monochromatic condition. This finding supports existing spatial research examining the role of experiential factors like early spatial activity experience in enhancing spatial skill. Encouraging female youth to engage in particular types of spatial activity experiences could help bridge the gap in spatial ability between the sexes. Spatial ability relates to future Science, Technology, Engineering and Mathematics (STEM) success (Shea et al., 2001; Wai et al., 2009) and addressing the factors that contribute to sex differences in spatial ability is of paramount importance. The lack of a significant relation between early spatial activity experience and mental rotation performance in the non-spatial cue conditions (i.e. facilitating cue and inhibiting cue conditions) shifts the focus from the development of an optimum cognitive strategy enhanced by early spatial activity experience towards future research aimed at understanding the development of strategy flexibility. One plausible explanation is that early spatial experience helps in the development of cognitive strategies that can be used across different spatial tasks. 
However, prolonged or current engagement with spatial content may facilitate strategy flexibility. The strategy questionnaire used in the current dissertation was based on retrospective participation in spatial activities and hence could not be used to investigate the role of prolonged or current spatial engagement in mental rotation performance and cognitive strategy flexibility.

Study III examined the developmental role of early spatial activity experience in the selection of an optimum cognitive strategy to enhance mental rotation performance. Results suggest a significant relation between early spatial activity experiences, specifically male sex-typed spatial activities and the optimum cognitive strategy in the monochromatic condition. This finding should act as a beacon against the stereotyping of spatial activities. Males are likely to spend more time in male sex-typed spatial activities than females, which could possibly explain the underperformance of women in spatial tasks.

\section{Cognitive Strategy Flexibility}

Study III examined the relation between cognitive strategy flexibility, mental rotation performance and early spatial activity experience. The number of changepoints detected using the PELT algorithm was used as an indicator of cognitive strategy flexibility. Results suggest a strong positive correlation between cognitive strategy flexibility and mental rotation performance. This strongly supports the argument that an optimum cognitive strategy may exist for a single type of stimulus, but may vary across stimuli depending on the amount and type of non-spatial information. Results also suggest that even though early spatial activity experience may play a developmental role 
in cognitive strategy selection, it may require prolonged or current engagement in spatial activities to influence cognitive strategy flexibility.

\section{Implications}

The implications of findings of the current dissertation are two fold.

Theoretical Implications. From a cognitive perspective, the latent variable of cognitive strategy selection composed of distinct eye movement patterns and its relation to mental rotation performance can help us in understanding inter-individual changes in cognitive strategy and intra-individual changes in cognitive strategy over time and task complexity. Spatial literature has consistently shown a sex difference in mental rotation performance with males outperforming females (Nazareth et al., 2013). With recent research linking success in science, technology, engineering and mathematics (STEM) disciplines to spatial ability (Lubinski, 2010; Wai et al., 2009), the need to close the gender gap has become more urgent. So far, interventions to improve spatial performance have worked equally well for men and women. However, given the finding that the type of non-spatial information affects male and female participants differently, we may be on the threshold of finding a way in which to close the gap between male and female mental rotation performance. Education in STEM fields may incorporate additional features in their educational pedagogy that helps female learners to grasp concepts that have seemed elusive in the past. While mental rotation research has primarily examined sex differences, the examination of raw eye-gaze streams, in the current dissertation, acts as exploratory analyses to discover new groupings of data based on previously unexamined or less frequently examined variables like early spatial activity experience, spatial 
anxiety, gender-role, etc. In addition, the raw eye-gaze plots create a uniform database of eye movement data, free of interpretation and fixation filter bias.

From a developmental standpoint, the current dissertation sets the foundation for the argument that cognitive strategy selection is the mechanism that develops as a consequence of spatial experience. Results suggest differences in cognitive strategy and performance as a function of early spatial experience and therefore it would be beneficial to track the development of these cognitive strategies over the entire lifespan and across populations with specific developmental delays. The findings of Quaiser-Pohl and Lehmann (2010) suggest that around the age of 6 years, children transition to using either a holistic or an analytical approach to mental rotation. Children younger than 6 years have been found to mentally rotate (Estes, 1998; Marmor, 1975; 1977), but there has been little research studying the cognitive strategy selected for mental rotation and the role of early spatial activity on the development of these strategies. Thus, the findings of the current dissertation can be extended beyond the undergraduate population in future studies. The early spatial experience of the current adult undergraduate sample is based on retrospective self-report of recalled childhood activities, which may not be an accurate reflection of their spatial experiences. This error can be greatly reduced by examining early spatial experience in younger populations.

Methodological Implications. Advances in technology have helped us use indirect measures like eye movement to track cognitive processes. However, like the initial stages of any new technology, researchers using eye tracking still grapple with conflicting interpretations of the data recorded by different eye-trackers, using different fixation filters. Although the volume of data ensures high statistical power, analyses on 
eye tracking parameters can be interpreted in several ways. The present methodological study, advocates for the use of raw eye-gaze plots to standardize eye-tracking findings across research. This provides a visual image of eye movement, which can then be categorized into different streams of data based on their temporal properties, free of investigator bias. In conclusion, this line of research aims at advancing our ability to link eye movement to cognitive processes while also enhancing our ability to manage and interpret large volumes of data.

\section{Future Directions}

Development of cognitive strategies in younger populations. This dissertation investigates the role of experiential factors on mental rotation performance through the development of an optimum cognitive strategy. Based on the findings, it would be interesting to track the development of these cognitive strategies in younger children. Children as young as 6 years have been found to mentally rotate (Estes, 1998; Marmor, $1975 ; 1977)$, but there has been little research studying the cognitive strategy selected for mental rotation and the role of early spatial activity on the development of these strategies. Future research can look at the role of different experiential factors like engagement in spatial activities, spatial language input, classroom/educational design, spatial toys, videogames etc. on the development of cognitive strategies across different age groups.

Development of effective spatial training techniques. In addition to engagement in spatial activities, spatial training has been found to be an effective way of improving mental rotation ability, thus suggesting malleability of spatial ability. Hoyek et al. (2009) found that mental rotation training sessions improved mental rotation test 
performance, which in turn was transferred to anatomy learning. Similarly, Wright et al. (2008) found a transfer of practice gains to novel practiced stimuli as well as novel nonpracticed stimuli thus concluding that practice effects were process based and not instance based. In Moreau (2013), 2D training was found to lead to an improvement in 2D tasks while 3D training was found to improve performance on 3D tasks. In addition, gender differences were found to disappear after 3D videogame training. Spence, $\mathrm{Yu}$, Feng, and Marshman (2009) found that the learning curve for women was similar to men when learning a basic spatial skill and hence training could help improve spatial performance in females. Uttal et al. (2013) present a meta-analysis of studies investigating the magnitude and generalizability of training on spatial skills. They concluded that training effects were stable and transferable to other spatial tasks that participants had not been trained on. However, these training studies raise important questions about the malleability of spatial cognition. For example, are there sex differences in responsiveness to spatial training? How much training is required to improve and sustain spatial performance? To be able to answer these questions, it is imperative to first investigate the mechanism (i.e. hypothesized as cognitive strategy selection) that develops as a consequence of experiential factors. Research on spatial training should then look at the development of flexibility in cognitive strategies as a result of different types and intensities of spatial training.

Gender Role Stereotyping. In recent years, the lines marking traditional gender roles have become blurred (Auster \& Ohm 2000; Hyde \& Lindberg, 2007). This suggests that an individual's gender role rather than their biological sex could be a more accurate predictor of their performance on spatial tasks. Individuals who identify with a more 
masculine gender role could potentially outperform those who identify with a more feminine gender role, even after controlling for participant sex. In their meta-analysis, Reilly and Neumann (2013) discuss gender-role mediation as a possible explanation for sex differences in mental rotation ability. They discuss studies in which researchers examined gender role of the subjects rather than their biological gender to study sex differences in mental rotation. Nash (1979) elaborates on the different mechanisms by which gender-role identification could influence spatial performance and these include gender-role conformity and self-efficacy beliefs. The proposed analysis in this dissertation includes gaze patterns based on performance and reported gender. However, future research using gender role identity, as a third comparison category would provide further insight into cognitive strategy selection.

\section{Limitations}

Statistical Analyses. In latent profile analyses, after selecting the preferred model for each experimental condition, the individual participant's posterior probabilities for each latent class were exported. Logistic regressions and ANOVAs were run to determine the relation between the latent variable of cognitive strategy selection and other variables of interest like mental rotation performance, participant sex and early spatial activity experience. This strategy may introduce some amount of error and consequently decrease precision (Berlin et al., 2014). However, the alternative was to use probability-based multiple imputation or pseudo class draws (Asparouhov \& Muthen, 2007), which has also been recently criticized (Lanza, Tan, \& Bray, 2013).

Statistical Power. The sample population in the current dissertation was recruited from undergraduate psychology courses, which typically have a higher proportion of 
females as compared to males. This was a major limitation of the dissertation as there was not sufficient statistical power to detect sex differences in some of the statistical analyses. 


\section{REFERENCES}

Akaike, H. (1987). Factor analysis and AIC. Psychometrika, 52(3), 317-332. doi:10.1007/BF02294359.

Alington, D. E., Leaf, R. C., \& Monaghan, J. R. (1992). Effects of Stimulus Color, Pattern, and Practice on Sex Differences in Mental Rotations Task Performance. The Journal of Psychology, 126(5), 539-553. doi:10.1080/00223980.1992.10543387

Ark, W. S. (2002). Neuroimaging studies give new insight to mental rotation (pp. 18221828). Presented at the 35th Annual Hawaii International Conference on System Sciences, IEEE Comput. Soc. doi:10.1109/HICSS.2002.994096

Aslin, R. N. (2012). Infant eyes: A window on cognitive development. Infancy, 17(1), 126140. doi:http://dx.doi.org/10.1111/j.1532-7078.2011.00097.x

Asparouhouv, T., \& Muthén, B. O. (2007). Wald test of mean equality for potential latent class predictors in mixture modeling. Retrieved November, 9, 2009.

Auster, C. J., \& Ohm, S. C. (2000). Masculinity and femininity in contemporary American society: A reevaluation using the Bem Sex-Role Inventory. Sex Roles, 43(7), 499528.

Baenninger, M., \& Newcombe, N. (1995). Environmental input to the development of sexrelated differences in spatial and mathematical ability. Learning and Individual Differences, 7(4), 363-379.

Baenninger, M., \& Newcombe, N. (1989). The role of experience in spatial test performance: A meta-analysis. Sex roles, 20(5-6), 327-344.

Baron, R. M., \& Kenny, D. A. (1986). The moderator-mediator variable distinction in social psychological research: Conceptual, strategic, and statistical considerations. Journal of personality and social psychology, 51(6), 1173.

Bem, S. L. (1974). The measurement of psychological androgyny. Journal of Consulting 
and Clinical Psychology, 42, 155-162.

Berlin, K. S., Williams, N. A., \& Parra, G. R. (2014). An introduction to latent variable mixture modeling (part 1): Overview and cross-sectional latent class and latent 
profile analyses. Journal of pediatric psychology, 39(2), 174-187.

Cherney, I. D., \& Voyer, D. (2010). Development of a Spatial Activity Questionnaire I: Items Identification - Springer. Sex Roles, 62(1-2), 89-99.

Cherney, I. D. (2008). Mom, let me play more computer games: They improve my mental rotation skills. Sex Roles, 59(11-12), 776-786. doi:http://dx.doi.org/10.1007/s11199008-9498-z

Cherney, I. D., \& London, K. (2006). Gender-linked Differences in the Toys, Television Shows, Computer Games, and Outdoor Activities of 5- to 13-year-old Children Springer. Sex Roles.

Clements-Stephens, A. M., Rimrodt, S. L., \& Cutting, L. E. (2009). Developmental sex differences in basic visuospatial processing: differences in strategy use? Neuroscience Letters, 449(3), 155-160. doi:10.1016/j.neulet.2008.10.094

Doyle, R. A., Voyer, D., \& Cherney, I. D. (2012). The relation between childhood spatial activities and spatial abilities in adulthood. Journal of Applied Developmental Psychology, 33(2), 112-120.

Duchowski, A. (2007). Eye tracking methodology: Theory and practice (Vol. 373). Springer Science \& Business Media.

Estes, D. (1998). Young Children's Awareness of Their Mental Activity: The Case of Mental Rotation. Child Development, 69(5), 1345-1360.

Gaulin, S. J., \& Hoffman, H. A. (1988). Evolution and development of sex differences in spatial ability. Human reproductive behaviour: a Darwinian perspective, 129-152.

Gibson, W. A. (1959). Three multivariate models: Factor analysis, latent structure analysis, and latent profile analysis. Psychometrika, 24(3), 229-252.

Ginn, S. R., \& Pickens, S. J. (2005). Relationships between spatial activities and scores on the mental rotation test as a function of sex. Perceptual and Motor Skills, 100(3), 877-881. doi:http://dx.doi.org/10.2466/PMS.100.3.877-881 
Guillot, A., Champely, S., Batier, C., Thiriet, P., \& Collet, C. (2006). Relationship Between Spatial Abilities, Mental Rotation and Functional Anatomy Learning. 
Advances in Health Sciences Education, 12(4), 491-507. doi:10.1007/s10459-0069021-7

Hausmann, M., Slabbekoorn, D., Van Goozen, S. H., Cohen-Kettenis, P. T., \& Güntürkün, O. (2000). Sex hormones affect spatial abilities during the menstrual cycle. Behavioral neuroscience, 114(6), 1245.

Hayes, A. F. (2012). PROCESS: A versatile computational tool for observed variable mediation, moderation, and conditional process modeling.

Hayes, A. F. (2009). Beyond Baron and Kenny: Statistical mediation analysis in the new millennium. Communication monographs, 76(4), 408-420.

Hegarty, M. (1992). Mental animation: Inferring motion from static displays of mechanical systems. Journal of Experimental Psychology: Learning, Memory, and Cognition, 18(5), 1084. doi:10.1037/0278-7393.18.5.1084

Hegarty, M. (2010). Components of Spatial Intelligence. In Psychology of Learning and Motivation (Vol. 52, pp. 265-297). Elsevier. doi:10.1016/S0079-7421(10)52007-3

Hugdahl, K., Thomsen, T., \& Ersland, L. (2006). Sex differences in visuo-spatial processing: An fMRI study of mental rotation. Neuropsychologia, 44(9), 1575-1583.

Hyde, J. S. (1990). Meta-Analysis and the Psychology of Gender Differences. Signs, 16(1), $55-73$.

Hyde, J. S., Lindberg, S. M., \& Wilson, A. (2007). Facts and assumptions about the nature of gender differences and the implications for gender equity. Handbook for achieving gender equity through education, 2, 19-32.

Jordan, K., Wüstenberg, T., Heinze, H. J., Peters, M., \& Jäncke, L. (2002). Women and men exhibit different cortical activation patterns during mental rotation tasks. Neuropsychologia, 40(13), 2397-2408.

Just, M. A., \& Carpenter, P. A. (1976). Eye fixations and cognitive processes. Cognitive Psychology, 8(4), 441-480. doi:10.1016/0010-0285(76)90015-3 
Khooshabeh, P., \& Hegarty, M. (2008). How visual information affects a spatial task. Presented at the Proceedings of Cognitive Science Society

Khooshabeh, P., Hegarty, M., \& Shipley, T. F. (2012). Individual Differences in Mental Rotation. Experimental Psychology (Formerly Zeitschrift Für Experimentelle Psychologie), 60(3), 164-171. doi:10.1027/1618-3169/a000184

Killick, R., Fearnhead, P., \& Eckley, I. A. (2012). Optimal detection of changepoints with a linear computational cost. Journal of the American Statistical Association, 107(500), 1590-1598.

Kozhevnikov, M. (2007). Cognitive styles in the context of modern psychology: Toward an integrated framework of cognitive style. Psychological Bulletin, 133(3), 464. doi:10.1037/0033-2909.133.3.464

Kozhevnikov, M., Hegarty, M., \& Mayer, R. E. (2002). Revising the Visualizer-Verbalizer Dimension: Evidence for Two Types of Visualizers. Cognition and Instruction, 20(1), 47-77.

Lawton, C. A. (1994). Gender differences in way-finding strategies: Relationship to spatial ability and spatial anxiety. Sex Roles, 30(11-12), 765-779. doi:10.1007/BF01544230

Lawton, C. A., \& Kallai, J. (2002). Gender Differences in Wayfinding Strategies and Anxiety About Wayfinding: A Cross-Cultural Comparison. Sex Roles, 47(9-10), 389-401. doi:10.1023/A:1021668724970

Lanza, S. T., Tan, X., \& Bray, B. C. (2013). Latent class analysis with distal outcomes: A flexible model-based approach. Structural equation modeling: a multidisciplinary journal, 20(1), 1-26.

Lei, M., \& Lomax, R. G. (2005). The effect of varying degrees of nonnormality in structural equation modeling. Structural Equation Modeling, 12(1), 1-27.

Linn, M. C., \& Petersen, A. C. (1985). Emergence and Characterization of Sex Differences in Spatial Ability: A Meta-Analysis. Child Development, 56(6), 1479-1498.

Lippa, R. A., Collaer, M. L., \& Peters, M. (2009). Sex Differences in Mental Rotation and Line Angle Judgments Are Positively Associated with Gender Equality and 
Economic Development Across 53 Nations. Archives of Sexual Behavior, 39(4), 990-997. doi:10.1007/s10508-008-9460-8

Lo, Y., Mendell, N. R., \& Rubin, D. B. (2001). Testing the number of components in a normal mixture. Biometrika, 88(3), 767-778.

Logie, R. H., Pernet, C. R., Buonocore, A., \& Sala, Della, S. (2011). Low and high imagers activate networks differentially in mental rotation. Neuropsychologia, 49(11), 30713077. doi:10.1016/j.neuropsychologia.2011.07.011

Lubinski, D. (2010). Spatial ability and STEM: A sleeping giant for talent identification and development. Personality and Individual Differences, 49(4), 344-351. doi:10.1016/j.paid.2010.03.022

Maccoby, E. E., \& Jacklin, C. N. (Eds.). (1974). The psychology of sex differences (Vol. 1). Stanford University Press.

MacKinnon, D. P., Lockwood, C. M., \& Williams, J. (2004). Confidence limits for the indirect effect: Distribution of the product and resampling methods. Multivariate behavioral research, 39(1), 99-128.

Marmor, G. S. (1975). Development of kinetic images: When does the child first represent movement in mental images? Cognitive Psychology, 7(4), 548-559. doi:10.1016/0010-0285(75)90022-5

Marmor, G. S. (1977). Mental rotation and number conservation: Are they related? Developmental Psychology, 13(4), 320. doi:10.1037/0012-1649.13.4.320

McLachlan, G. J., \& Peel, D. (2000). Finite mixture modelsWiley. New York.

Moè, A., Meneghetti, C., \& Cadinu, M. (2009a). Women and mental rotation: Incremental theory and spatial strategy use enhance performance. Personality and Individual Differences, 46(2), 187-191. doi:10.1016/j.paid.2008.09.030

Moreau, D. (2013). Differentiating two- from three-dimensional mental rotation training effects. The Quarterly Journal of Experimental Psychology, 66(7), 1399-1413. doi:10.1080/17470218.2012.744761 
Muthén, B., \& Asparouhov, T. (2003). Modeling interactions between latent and observed continuous variables using maximum-likelihood estimation in Mplus. Mplus Web Notes, 6, 1-9.

Nash, S. C. (1975). The relationship among sex-role stereotyping, sex-role preference, and the sex difference in spatial visualization. Sex Roles, 1(1), 15-32.

Nazareth, A., Herrera, A., \& Pruden, S. M. (2013). Explaining sex differences in mental rotation: role of spatial activity experience. Cognitive Processing, 14(2), 201-204. doi:10.1007/s10339-013-0542-8

Neidhardt, E., \& Popp, M. (2010). Spatial tests, familiarity with the surroundings, and spatial activity experience: How do they contribute to children's spatial orientation in macro environments?. Journal of Individual Differences, 31(2), 59.

Newcombe, N., Bandura, M. M., \& Taylor, D. G. (1983). Sex differences in spatial ability and spatial activities. Sex Roles, 9(3), 377-386. doi:10.1007/BF00289672

Nylund, K. L., Asparouhov, T., \& Muthén, B. O. (2007). Deciding on the number of classes in latent class analysis and growth mixture modeling: A Monte Carlo simulation study. Structural equation modeling, 14(4), 535-569.

Pylyshyn, Z. (2003). Return of the mental image: are there really pictures in the brain? Trends in Cognitive Sciences.

Quaiser-Pohl, C., Rohe, A. M., \& Amberger, T. (2010). The solution strategy as an indicator of the developmental stage of preschool children's mental-rotation ability. Journal of Individual Differences, 31(2), 95. doi:10.1027/1614-0001/a000017

Reilly, D., \& Neumann, D. L. (2013). Gender-Role Differences in Spatial Ability: A MetaAnalytic Review. Sex Roles, 68(9-10), 521-535. doi:10.1007/s11199-013-0269-0

Schwarz, G. (1978). Estimating the dimension of a model. The annals of statistics, 6(2), 461-464.

Schwartz, D. (1996). Shuttling between depictive models and abstract rules: Induction and fallback. Cognitive Science, 20(4), 457-497. doi:10.1016/S0364-0213(99)80012-3 
Sclove, S. L. (1987). Application of model-selection criteria to some problems in multivariate analysis. Psychometrika, 52(3), 333-343.

Shea, D. L., Lubinski, D., \& Benbow, C. P. (2001). Importance of assessing spatial ability in intellectually talented young adolescents: A 20-year longitudinal study. Journal of Educational Psychology, 93(3), 604.

Shepard, R. N., \& Metzler, J. (1971). Mental rotation of three-dimensional objects.

Sherman, J. A. (1967). Problem of sex differences in space perception and aspects of intellectual functioning. Psychological Review, 74(4), 290. doi:10.1037/h0024723

Sherman, J. A. (1974). Field articulation, sex, spatial visualization, dependency, practice, laterality of the brain and birth order. Perceptual and Motor Skills, 38(3c), 12231235.

Signorella, M. L., Krupa, M. H., Jamison, W., \& Lyons, N. (1986). A short version of a spatial activity questionnaire - Springer. Sex Roles, 14(9-10), 475-479.

Silverman, I., Kastuk, D., Choi, J., \& Phillips, K. (1999). Testosterone levels and spatial ability in men. Psychoneuroendocrinology, 24(8), 813-822.

Spence, I., Yu, J. J., Feng, J., \& Marshman, J. (2009). Women match men when learning a spatial skill. Journal of Experimental Psychology: Learning, Memory, and Cognition, 35(4), 1097-1103. doi:10.1037/a0015641

Stieff, M. (2007). Mental rotation and diagrammatic reasoning in science. Learning and Instruction, 17(2), 219-234. doi:10.1016/j.learninstruc.2007.01.012

Terlecki, M. S., \& Newcombe, N. S. (2005). How important is the digital divide? the relation of computer and videogame usage to gender differences in mental rotation 
ability. Sex Roles, 53(5-6), 433-441. doi:http://dx.doi.org/10.1007/s11199-005-67650

Terlecki, M. S., Newcombe, N. S., \& Little, M. (2008). Durable and generalized effects of spatial experience on mental rotation: gender differences in growth patterns. Applied Cognitive Psychology, 22(7), 996-1013. doi:10.1002/acp.1420

Uttal, D. H., Meadow, N. G., Tipton, E., Hand, L. L., Alden, A. R., Warren, C., \& Newcombe, N. S. (2013). The malleability of spatial skills: A meta-analysis of training studies. Psychological Bulletin, 139(2), 352. doi:10.1037/a0028446

Vandenberg, S. G., \& Kuse (1978). Mental rotations, a group test of three dimensional spatial visualization. Perceptual and Motor Skills, 47(2), 599-604.

doi:10.2466/pms.1978.47.2.599

Voyer, D., Nolan, C., \& Voyer, S. (2000). The relation between experience and spatial performance in men and women. Sex Roles,43(11-12), 891-915. doi:http://dx.doi.org/10.1023/A:1011041006679

Voyer, D., Voyer, S., \& Bryden, M. P. (1995). Magnitude of sex differences in spatial abilities: A meta-analysis and consideration of critical variables. Psychological Bulletin, 117(2), 250. doi:10.1037/0033-2909.117.2.250

Wai, J., Lubinski, D., \& Benbow, C. P. (2009). Spatial ability for STEM domains: Aligning over 50 years of cumulative psychological knowledge solidifies its importance. Journal of Educational Psychology, 101(4), 817. doi:10.1037/a0016127

Wright, R., Thompson, W. L., Ganis, G., Newcombe, N. S., \& Kosslyn, S. M. (2008). Training generalized spatial skills. Psychonomic Bulletin \& Review, 15(4), 763-771. doi:10.3758/PBR.15.4.763

Yu, C., Yurovsky, D., \& Xu, T. (2011). Visual Data Mining: An Exploratory Approach to Analyzing Temporal Patterns of Eye Movements. Infancy, 17(1), 33-60. doi:10.1111/j.1532-7078.2011.00095.x

Zucker, K. J., Mitchell, J. N., Bradley, S. J., Tkachuk, J., Cantor, J. M., \& Allin, S. M. (2006). The Recalled Childhood Gender Identity/Gender Role Questionnaire: 
Psychometric Properties. Sex Roles, 54(7-8), 469-483. doi:10.1007/s11199-0069019-x 


\section{Appendix A}

Tobii X60 Eye Tracker Specifications and Terminology

Technical Specifications for Tobii X60 eye trackers (reproduced from the Tobii X60 technical manual)

\begin{tabular}{|c|c|}
\hline Technical Specifications & Tobii X60 \\
\hline Dimensions (lXbXh) & $320 \mathrm{~mm}$ X $163 \mathrm{~mm}$ X $85 \mathrm{~mm}$ \\
\hline Data Rate & $60 \mathrm{~Hz}$ \\
\hline Accuracy & Typical 0.5 degrees \\
\hline Spatial Resolution & Typical 0.2 degrees \\
\hline Head Movement Error & Typical 0.2 degrees \\
\hline Tracking Distance & $50-80 \mathrm{~cm}$ \\
\hline Top head-motion speed & $25 \mathrm{~cm} / \mathrm{second}$ \\
\hline
\end{tabular}


Definitions

(Reproduced from the Tobii Studio 3.2 user manual) A mathematical and statistical process by which raw data is parsed into fixations based on the velocity (in visual degrees per

Fixation second) of the directional shifts of the eye. If the velocity of the eye movement is below a certain threshold the samples are classified as part of a fixation.

Fixation Duration

Fixation Count

Visit

Visit Duration

Visit Count

Percentage

Fixated
Measures the duration of each individual fixation within an AOI.

Measures the number of times the participant fixates on an AOI

The interval of time between the first fixation on the AOI and the next fixation outside the AOI.

Measures the duration of each individual visit within an AOI

Measures the number of visits within an active AOI

Measures the number of recordings in which participants have fixated at least once within an AOI or AOI group and expresses it as a fraction of the total number of recordings 


\section{Appendix $B$}

\section{Verbal Protocol}

Participants were asked a series of questions in a fixed sequence at the end of the eye tracking study component.

1. "How did you decide whether the two figures were a match or a non-match?"

2. "Which figure did you generally choose to rotate? The one to the right or the one to the left?"

3. Which hand do you predominantly use for writing purposes?"

4. "Did you use the red cube in any way while making your decision?"

Note: Question 2 was asked only if a participant used the words "rotate", "flip", etc., to indicate rotation in response to question 1. Question 4 was asked only if a participant was either in the facilitating cue (FC) or the inhibiting cue (IC) conditions and had not mentioned the red cube in question 1. Although question 3 was asked to examine handedness and its role in mental rotation, it is beyond the scope of the current dissertation. 


\section{Measures}

\section{Spatial Anxiety Scale (Lawton \& Kallai, 2002).}

Instruction: Below is a list of scenarios that we would like for you to imagine.

Please indicate how much you are bothered by each scenario.

1. Deciding which direction to walk in an unfamiliar city or town after coming out of a train/bus/metro station OR parking garage.

2. Finding my way to an appointment in an unfamiliar area of a city or town.

3. Leaving a store that I have been to for the first time and deciding which way to turn to get to a destination.

4. Finding my way back to a familiar area after realizing I have made a wrong turn and become lost while travelling.

5. Finding my way in an unfamiliar shopping mall, medical center, or large building complex.

6. Finding my way out of a complex arrangement of offices that I have visited for the first time.

7. Trying a new route that I think will be a shortcut, without a map.

8. Pointing in the direction of a place outside that someone wants to get to and has asked for directions, when I am in a windowless room.

9. When driving south, quickly deciding which way to turn to head east.

10. In a classroom, being asked to answer the following: John is taller than Mary, and Mary is taller than Chris. Who is shorter, John or Chris?

11. Being asked to point to a foreign country on a map.

12. Being asked to give directions from school to my house. 
13. Working out how much change a cashier should have given me after buying several items.

14. Calculating how many days until a person's birthday.

15. Calculating what year a 15 -year-old would have been born.

16. Locating my car in a very large parking lot or parking garage.

Note: The spatial anxiety scale ranged from 1 - 'not at all' to 4 - 'Severely. I could barely stand it'. 
Strategy Questionnaire (Moè, Meneghetti, \& Cadinu, 2009b).

Instruction: Think back to the test that you took. Rate how much you used each strategy below ( 0 indicating that you did not use this strategy and 7 indicating that you heavily implemented this strategy):

1. I rotated the stimulus on the left to match the stimulus on the right.

2. I rotated the stimulus on the right to match the stimulus on the left.

3. I rotated the stimulus as a single whole object.

4. I imagined myself rotating around the object.

5. I counted the cubes.

6. I rotated the stimulus piece by piece. 


\section{Spatial Activity Survey (Newcombe et al., 1983).}

We would like you to indicate the degree to which you have participated in each of the following 81 activities. Next to each activity, there is a scale ranging from 1 (never participated) to 6 (participated more than once a week). Please mark the number, which best corresponds to your level of participation for each activity. For the purpose of this survey please indicate your participation in each of these activities from preschool (3years-old) through adolescence (18-years-old).

\section{List of Activities according to sex-type.}

Note: In the actual survey, activities were presented to the participant in random order.

\begin{tabular}{lll}
\hline Masculine & Neutral & Feminine \\
\hline Touch Football & Bowling & Figure Skating \\
Tackle Football & Softball & Field hockey \\
Baseball & Advanced tennis & Baton Twirling (toss in air) \\
Basketball & Ping pong & Baton twirling ( $>$ 1 baton) \\
Ice Hockey & Volleyball & Water ballet \\
Advanced Racquetball & Beginning racquetball & Gymnastics \\
Soccer & Dodge ball & Ballet (pirouettes) \\
Squash & Jumping horses & Ballet (choreography) \\
Darts & Driving & Tap Dance (own routine) \\
Horseshoes & Frisbee & Disco dancing (with falls) \\
Archery & Jewelry (mount stones) & Pottery (wheel) \\
Golf & Drawing (three- & Embroidery (no pattern) \\
Hunting & dimensional) & Crochet (with seams) \\
Target Shooting & Painting (3 dimensional) & Knitting (with seams) \\
Rock Climbing & Leatherwork (with seams) & Knitting (multicolor) \\
Canoeing (shooting rapids) & Sculpting & Quilting \\
Sledding (around & wrap) & \\
obstacles) & Photography & Tailoring \\
Skiing & Navigate in car & Arranging furniture \\
Skiing (jumping) & Layout for newspaper, & Touch Typing \\
Skateboarding & Marching band & Interior Decorating \\
Fencing & & Sketch Clothes Designs \\
High Jumping & & \\
Pole Vaulting & & \\
& & \\
& &
\end{tabular}


Shooting Pool

Foosball

Air Hockey

Glass Blowing

Building model planes

Building train or racecar

sets

Building go-carts

Juggling

Mechanical drawing

Car Repair

Electrical Circuitry

Plumbing

Carpentry

Make/fix radios, stereos

Sketch auto designs

Sketch house plans

Using compass 


\section{TABLES}

Table 1

Summary Statistics of Participant Demographic Information for Study I and III

\begin{tabular}{|c|c|c|c|c|}
\hline & & Monochromatic & $\begin{array}{l}\text { Facilitating Cue } \\
\text { Condition }\end{array}$ & $\begin{array}{c}\text { Inhibiting Cue } \\
\text { Condition }\end{array}$ \\
\hline \multirow{3}{*}{ Sample Size } & Female & $112(68.3 \%)$ & $73(78.5 \%)$ & $93(71.5 \%)$ \\
\hline & Male & $52(31.7 \%)$ & $20(21.5 \%)$ & $37(28.5 \%)$ \\
\hline & Total & 164 & 93 & 130 \\
\hline \multirow{3}{*}{ Ethnicity } & Hispanic & $111(67.7 \%)$ & $60(64.5 \%)$ & $72(55.4 \%)$ \\
\hline & Other & $53(32.3 \%)$ & $31(33.3 \%)$ & $51(39.2 \%)$ \\
\hline & White & $102(62.2 \%)$ & $61(65.6 \%)$ & $75(57.7 \%)$ \\
\hline \multirow{3}{*}{ Race } & $\begin{array}{c}\text { Africa- } \\
\text { American }\end{array}$ & $24(14.6 \%)$ & $20(21.5 \%)$ & $27(20.8 \%)$ \\
\hline & Asian & $13(7.9 \%)$ & $4(4.3 \%)$ & $12(9.2 \%)$ \\
\hline & Other & $32(19.5 \%)$ & $10(10.8 \%)$ & $10(7.7 \%)$ \\
\hline
\end{tabular}


Table 2

Coding scheme for Video Protocol

Holistic Strategy (categorical 1)

"I rotated figure/image..."

"I flipped figure/image..."

"I turned figure/image..."

"I tilted whole figure/image..."

"I switched one figure to the same position..."

"I reversed figure positions..."
Piecemeal Strategy (categorical 2)

"I counted cubes in figure/image..."

"I counted lines in one figure/image..."

"I looked at angles..."

"I traced lines..."

"I looked at the top arm..."

Note. If participants used a combination of holistic and piecemeal strategy words to describe his/her decision making process, it was categorized as a mixed strategy (categorical 3) 
Table 3

Descriptive Statistics of Participant Demographic Information for Study I and III

\begin{tabular}{ccccc} 
& & Monochromatic & $\begin{array}{c}\text { Facilitating Cue } \\
\text { Condition }\end{array}$ & $\begin{array}{c}\text { Inhibiting Cue } \\
\text { Condition }\end{array}$ \\
\hline Age & M (SD) & $21.16(2.75)$ & $20.51(2.58)$ & $20.50(1.93)$ \\
GPA & M (SD) & $3.11(0.55)$ & $3.20(0.58)$ & $3.13(0.58)$ \\
Spatial Anxiety & M (SD) & $27.89(6.60)$ & $29.67(6.53)$ & $29.92(7.54)$ \\
General Anxiety & M (SD) & $33.18(13.03)$ & $33.28(12.33)$ & $35.11(14.42)$ \\
\hline
\end{tabular}


Table 4

Descriptive Statistics for Study Variables

\begin{tabular}{|c|c|c|c|}
\hline Variable Name & Mean (SD) & Skewness & Kurtosis \\
\hline \multicolumn{4}{|l|}{ Monochromatic Condition } \\
\hline Completion Time & $9.11(3.30)$ & 1.17 & 1.35 \\
\hline MRT Score & $29.54(6.52)$ & 0.27 & 1.08 \\
\hline Fixation Duration & $0.23(0.06)$ & -0.48 & 0.55 \\
\hline Fixation Count & $12.38(6.30)$ & 0.78 & 0.66 \\
\hline Visit Duration & $0.75(0.33)$ & 0.19 & 0.38 \\
\hline Visit Count & $4.98(2.31)$ & 0.72 & 0.41 \\
\hline Spatial Activity & $1.56(0.35)$ & 0.74 & 0.15 \\
\hline Masculine Spatial Activity & $1.46(0.37)$ & 1.57 & 0.54 \\
\hline Feminine Spatial Activity & $1.48(.38)$ & 1.18 & 0.54 \\
\hline Neutral Spatial Activity & $1.81(.54)$ & 0.87 & 0.62 \\
\hline \multicolumn{4}{|l|}{ Facilitating Cue (FC) Condition } \\
\hline Completion Time & $8.48(2.90)$ & 0.90 & 0.61 \\
\hline MRT Score & $28.84(6.27)$ & -0.24 & -0.89 \\
\hline Fixation Duration & $0.25(0.05)$ & -0.49 & 0.96 \\
\hline Fixation Count & $3.88(1.75)$ & 1.17 & 1.57 \\
\hline Visit Duration & $0.36(0.08)$ & -0.04 & 0.43 \\
\hline Visit Count & $2.96(1.22)$ & 1.02 & 1.01 \\
\hline Spatial Activity & $1.62(0.27)$ & 0.14 & -0.31 \\
\hline Masculine Spatial Activity & $1.46(.24)$ & 0.32 & -0.27 \\
\hline Feminine Spatial Activity & $1.63(.42)$ & 0.81 & 0.53 \\
\hline Neutral Spatial Activity & $1.94(.44)$ & 0.27 & -0.14 \\
\hline \multicolumn{4}{|l|}{ Inhibiting Cue (IC) Condition } \\
\hline Completion Time (mins) & $8.75(3.46)$ & 0.60 & 0.21 \\
\hline MRT Score & $25.58(7.33)$ & 0.22 & -1.25 \\
\hline Fixation Duration & $0.24(0.07)$ & -0.05 & 0.35 \\
\hline Fixation Count & $3.68(1.76)$ & 0.88 & 0.55 \\
\hline Visit Duration & $0.34(0.10)$ & -0.14 & 1.30 \\
\hline Visit Count & $2.80(1.26)$ & 0.80 & 0.12 \\
\hline Spatial Activity & $1.63(0.36)$ & 0.56 & 0.23 \\
\hline Masculine Spatial Activity & $1.53(0.39)$ & 1.11 & 1.90 \\
\hline Feminine Spatial Activity & $1.55(0.46)$ & 0.95 & 0.41 \\
\hline Neutral Spatial Activity & $1.93(0.54)$ & 0.50 & -0.31 \\
\hline
\end{tabular}


Table 5

Comparison of Mental Rotation Performance Monochromatic

Facilitating Cue Inhibiting Cue Condition Condition

\begin{tabular}{lcccc} 
MRT Score & $\mathrm{M}$ & 29.54 & 28.84 & $* 25.58$ \\
& $\mathrm{SD}$ & 6.52 & 6.27 & 7.33 \\
\hline * Indicates significant difference in mental rotation performance from the other two \\
experimental conditions
\end{tabular}
experimental conditions 
Table 6

Sex Differences in Mental Rotation Performance

\begin{tabular}{lccccc}
\hline \multirow{2}{*}{ MRT Score } & \multicolumn{2}{c}{ Male } & \multicolumn{2}{c}{ Female } & Significance \\
\cline { 2 - 6 } & $\mathrm{M}$ & $\mathrm{SD}$ & $\mathrm{M}$ & $\mathrm{SD}$ & $\mathrm{p}$ \\
\hline Monochromatic & 31.98 & 5.72 & 28.42 & 6.59 & $* t(160)=3.32, d=0.58$ \\
Facilitating Cue (FC) & 30.20 & 6.25 & 28.47 & 6.26 & $\mathrm{t}(91)=1.10, \mathrm{NS}$ \\
Inhibiting Cue (IC) & 28.28 & 8.20 & 24.59 & 6.77 & $* \mathrm{t}(118)=2.49, \mathrm{~d}=.50$ \\
\hline
\end{tabular}

$$
* \mathrm{p}<.05
$$


Table 7.

Relation between Self-reported (Video Protocol) Cognitive Strategy Selection and Mental Rotation Performance

\begin{tabular}{lccc}
\hline Self-reported Strategy & $N$ & $\begin{array}{c}\text { Mean (MRT } \\
\text { Score) }\end{array}$ & $S D$ \\
\hline Monochromatic Condition & 94 & 31.14 & 5.85 \\
$\quad$ Holistic Strategy & 27 & 25.44 & 5.87 \\
Piecemeal Strategy & 32 & 28.13 & 7.41 \\
Mixed Strategy & & & \\
& & & \\
Facilitating Cue (FC) Condition & 59 & 30.19 & 5.89 \\
Holistic Strategy & 22 & 25.45 & 6.12 \\
Piecemeal Strategy & 8 & 28.75 & 6.18 \\
Mixed Strategy & & & \\
& & & \\
Inhibiting Cue (IC) Condition & 56 & 27.05 & 6.31 \\
Holistic Strategy & 36 & 23.00 & 6.24 \\
Piecemeal Strategy & 16 & 26.50 & 5.03 \\
Mixed Strategy & & & \\
\hline
\end{tabular}


Table 8 .

Mean Estimates of Each Item in the Strategy Questionnaire

\begin{tabular}{lll}
\multicolumn{1}{c}{ Strategy Questionnaire } & $\mathrm{M}$ & $\mathrm{SD}$ \\
\hline $\begin{array}{l}\text { Monochromatic Condition (N=162) } \\
\text { "I rotated the stimulus on the left to match the stimulus on the }\end{array}$ & 3.34 & 2.54 \\
right." & & \\
"I rotated the stimulus on the right to match the stimulus on & 4.31 & 2.48 \\
the left." & 3.94 & 2.44 \\
"I rotated the stimulus as a single whole object." & 2.59 & 2.80 \\
"I imagined myself rotating around the object." & 4.74 & 2.20 \\
"I counted the cubes." & 1.98 & 2.13 \\
"I rotated the stimulus piece by piece." & & \\
& & \\
Facilitating Cue (FC) Condition (N=93) & 3.13 & 2.58 \\
"I rotated the stimulus on the left to match the stimulus on the & & \\
right." \\
"I rotated the stimulus on the right to match the stimulus on \\
the left." \\
"I rotated the stimulus as a single whole object." \\
"I imagined myself rotating around the object." \\
"I counted the cubes." \\
"I rotated the stimulus piece by piece."
\end{tabular}


Table 9

Correlations between Items in the Strategy Questionnaire

\begin{tabular}{|c|c|c|c|c|c|c|}
\hline & 1 & 2 & 3 & 4 & 5 & 6 \\
\hline \multicolumn{7}{|l|}{ Monochromatic Condition $(N=162)$} \\
\hline $\begin{array}{l}\text { 1. "I rotated the stimulus on the left to } \\
\text { match the stimulus on the right." }\end{array}$ & 1 & $.39 * *$ & .08 & .10 & $.16^{*}$ & .01 \\
\hline $\begin{array}{l}\text { 2. "I rotated the stimulus on the right } \\
\text { to match the stimulus on the left." }\end{array}$ & - & 1 & .13 & -.04 & -.09 & .07 \\
\hline $\begin{array}{l}\text { 3. "I rotated the stimulus as a single } \\
\text { whole object." }\end{array}$ & - & - & 1 & .14 & .03 & .04 \\
\hline $\begin{array}{l}\text { 4. "I imagined myself rotating around } \\
\text { the object." }\end{array}$ & - & - & - & 1 & .09 & $.22 * *$ \\
\hline 5. "I counted the cubes." & - & - & - & - & 1 & $.30 * *$ \\
\hline $\begin{array}{l}\text { 6. "I rotated the stimulus piece by } \\
\text { piece." }\end{array}$ & - & - & - & - & - & 1 \\
\hline
\end{tabular}

Facilitating Cue (FC) Condition $(N=91)$

1. "I rotated the stimulus on the left to match the stimulus on the right."

2. "I rotated the stimulus on the right to match the stimulus on the left." 3. "I rotated the stimulus as a single whole object."

4. "I imagined myself rotating around the object."

5. "I counted the cubes."

6. "I rotated the stimulus piece by piece."

Inhibiting Cue (IC) Condition

$(N=120)$

1. "I rotated the stimulus on the left to match the stimulus on the right."

2. "I rotated the stimulus on the right to match the stimulus on the left." 3. "I rotated the stimulus as a single whole object."

4. "I imagined myself rotating around the object."

5. "I counted the cubes."

6 . "I rotated the stimulus piece by piece."

$\begin{array}{cccccc}1 & - & .19 & -.15 & .17 & .05 \\ - & 1 & -.05 & .13 & -.11 & .10 \\ - & - & 1 & .13 & .29 * * & .05 \\ - & - & - & 1 & -.11 & .14 \\ - & - & - & - & 1 & .02 \\ - & - & - & - & - & 1\end{array}$


Table 10

Fit Indices for the Latent Profile Analysis Models of Cognitive Strategy Selection during Mental Rotation

\begin{tabular}{|c|c|c|c|c|}
\hline No. Of Classes & 1 & 2 & 3 & 4 \\
\hline No. Of free parameters & 12 & 15 & 22 & 29 \\
\hline Monochromatic Condition & $N=162$ & & & \\
\hline LL & -1376.34 & -636.45 & -575.36 & -513.53 \\
\hline AIC & 2776.67 & 1302.90 & 1194.73 & 1085.06 \\
\hline $\mathrm{BIC}$ & 2813.87 & 1349.21 & 1262.65 & 1174.60 \\
\hline $\mathrm{ABIC}$ & 2775.88 & 1301.72 & 1193.00 & 1082.79 \\
\hline Entropy & $\mathrm{NA}^{\mathrm{c}}$ & .86 & .87 & .90 \\
\hline $\operatorname{LMR}(p)$ & $\mathrm{NA}^{\mathrm{c}}$ & $.03^{\mathrm{b}}$ & .38 & .24 \\
\hline $\operatorname{BLTR}(p)$ & $\mathrm{NA}^{\mathrm{c}}$ & .00 & .00 & .00 \\
\hline Facilitating Cue $(F C)$ & $N=93$ & & & \\
\hline Condition & & & & \\
\hline LL & -435.25 & -7.92 & 20.27 & 48.57 \\
\hline AIC & 894.49 & 45.85 & 3.47 & -39.14 \\
\hline $\mathrm{BIC}$ & 924.88 & 83.84 & 59.19 & 34.31 \\
\hline $\mathrm{ABIC}$ & 887.00 & 36.48 & -10.26 & -57.24 \\
\hline Entropy & $\mathrm{NA}^{\mathrm{c}}$ & .96 & .85 & .91 \\
\hline $\operatorname{LMR}(p)$ & $\mathrm{NA}^{\mathrm{c}}$ & $.01^{\mathrm{b}}$ & .77 & .25 \\
\hline $\operatorname{BLTR}(p)$ & $\mathrm{NA}^{\mathrm{c}}$ & .00 & .00 & .00 \\
\hline Inhibiting Cue (FC) & $N=121$ & & & \\
\hline Condition & & & & \\
\hline LL & -2096.05 & -923.79 & -591.45 & -274.47 \\
\hline AIC & 4216.10 & 1877.58 & 1226.90 & 606.94 \\
\hline $\mathrm{BIC}$ & 4249.94 & 1919.52 & 1288.41 & 688.02 \\
\hline $\mathrm{ABIC}$ & 4212.00 & 1872.09 & 1218.85 & 596.33 \\
\hline Entropy & $\mathrm{NA}^{\mathrm{c}}$ & 1 & 1 & 1 \\
\hline $\operatorname{LMR}(p)$ & $\mathrm{NA}^{\mathrm{c}}$ & .34 & .27 & .24 \\
\hline $\operatorname{BLTR}(p)$ & $\mathrm{NA}^{\mathrm{c}}$ & .00 & .00 & .00 \\
\hline
\end{tabular}

Note. $\mathrm{LL}=$ Log Likelihood; AIC = Akaike Information Criterion; ABIC = Adjusted BIC; LMR = Lo-Mendell-Rubin Adjusted Likelihood Ratio Test; BLRT = Bootstrap Likelihood Ratio Test. There were 4 indicators: fixation duration (FD), fixation count (FC), visit duration (VD) and visit count (VC).

${ }^{\mathrm{b}}$ Best-fitting model according to that index.

${ }^{\mathrm{c}}$ LMR and BLRT are not available for the one-class model. 
Table 11

Average Latent Class Probabilities for Most Likely Class Membership

Latent Class Model Latent Class membership

\begin{tabular}{|c|c|c|c|c|}
\hline \multirow{2}{*}{\multicolumn{5}{|c|}{$\begin{array}{l}\text { Monochromatic Condition } \\
\text { 2-class }\end{array}$}} \\
\hline & & & & \\
\hline \multicolumn{5}{|l|}{ Class $1(n=115)$} \\
\hline Class $2(n=47)$ & .03 & .93 & - & - \\
\hline \multicolumn{5}{|l|}{3 -class } \\
\hline Class $1(n=33)$ & .94 & .0 & .02 & - \\
\hline Class $2(n=27)$ & .00 & .93 & .03 & - \\
\hline Class $3(n=102)$ & .07 & .07 & .95 & - \\
\hline \multicolumn{5}{|l|}{ 4-class } \\
\hline Class $1(n=23)$ & .95 & .00 & .05 & .00 \\
\hline Class $2(n=34)$ & .00 & .91 & .10 & .00 \\
\hline Class $3(n=100)$ & .02 & .03 & .95 & .00 \\
\hline Class $4(n=5)$ & .00 & .01 & .00 & 1.00 \\
\hline \multicolumn{5}{|c|}{ Facilitating Cue (FC) Condition } \\
\hline \multicolumn{5}{|c|}{ 2-class } \\
\hline Class $1(n=76)$ & .99 & .01 & - & - \\
\hline Class $2(n=17)$ & .01 & .99 & - & - \\
\hline \multicolumn{5}{|l|}{3 -class } \\
\hline Class $1(n=24)$ & .90 & .02 & .06 & - \\
\hline Class $2(n=13)$ & .01 & .98 & .00 & - \\
\hline Class $3(n=56)$ & .10 & .00 & .94 & - \\
\hline \multicolumn{5}{|l|}{ 4-class } \\
\hline Class $1(\mathrm{n}=9)$ & .98 & .00 & .02 & .00 \\
\hline Class $2(n=24)$ & .00 & .94 & .04 & .00 \\
\hline Class $3(n=48)$ & .01 & .06 & .94 & .00 \\
\hline Class $4(n=12)$ & .01 & .00 & .00 & .99 \\
\hline \multicolumn{5}{|l|}{ Inhibiting Cue (IC) Condition } \\
\hline \multicolumn{5}{|l|}{ 2-class } \\
\hline Class $1(n=120)$ & 1.00 & .00 & - & - \\
\hline Class $2(n=1)$ & .00 & 1.00 & - & - \\
\hline \multicolumn{5}{|l|}{3 -class } \\
\hline Class $1(n=118)$ & 1.00 & .00 & .00 & - \\
\hline Class $2(n=1)$ & .00 & 1.00 & .00 & - \\
\hline Class $3(n=2)$ & .00 & .00 & 1.00 & - \\
\hline \multicolumn{5}{|l|}{ 4-class } \\
\hline Class $1(n=2)$ & 1.00 & .00 & .00 & .00 \\
\hline Class $2(n=2)$ & .00 & 1.00 & .00 & .00 \\
\hline Class $3(n=116)$ & .00 & .00 & 1.00 & .00 \\
\hline Class $4(n=1)$ & .00 & .00 & .00 & 1.00 \\
\hline
\end{tabular}


Table 12

Latent Class Means for Eye Movement Parameters in the 2-class Latent Model

\begin{tabular}{|c|c|c|}
\hline \multirow[b]{2}{*}{ Unstandardized means } & \multicolumn{2}{|c|}{ Latent Class } \\
\hline & 1 & 2 \\
\hline \multicolumn{3}{|l|}{ Monochromatic Condition* } \\
\hline Fixation Duration & .23 & .25 \\
\hline Fixation Count & 9.41 & 20.47 \\
\hline Visit Duration & .75 & .78 \\
\hline Visit Count & 3.86 & 7.92 \\
\hline \multicolumn{3}{|l|}{ Facilitating Cue (FC) Condition ${ }^{+}$} \\
\hline Fixation Duration & .25 & .29 \\
\hline Fixation Count & 3.21 & 6.82 \\
\hline Visit Duration & .34 & .42 \\
\hline Visit Count & 2.48 & 5.02 \\
\hline \multicolumn{3}{|l|}{ Inhibiting Cue (FC) Condition ${ }^{+}$} \\
\hline Fixation Duration & - & - \\
\hline Fixation Count & - & - \\
\hline Visit Duration & - & - \\
\hline Visit Count & - & - \\
\hline
\end{tabular}


Table 13

Logistic Regression Coefficients for the 2-class Latent Profile Analysis with Mental Rotation Score and Participant Sex as Latent Class Predictors

\begin{tabular}{lcc}
\hline & \multicolumn{2}{c}{ Latent Class } \\
\cline { 2 - 3 } Lonochromatic Condition & 1 & 2 \\
MRT Score & $-.232^{*}$ & $.232^{*}$ \\
Sex & .048 & -.048 \\
Facilitating Cue (FC) Condition & & \\
MRT Score & $-.177^{*}$ & $.177^{*}$ \\
Sex & .148 & -.148 \\
Inhibiting Cue (FC) Condition & & - \\
MRT Score & - & - \\
Sex & - & \\
\hline
\end{tabular}


Table 14

Latent Classes of Cognitive Strategy by Participant Sex

Latent Profiles based on Eye movement Parameters

Participant Sex Latent Class 1 Latent Class 2

Monochromatic Condition

$\left(\chi^{2}=1.43, \mathrm{p}>.05, \mathrm{df}=1\right)$

Female

$82(73.9 \%)$

$33(64.7 \%)$

$29(26.1 \%)$

Male

$60(82.2 \%)$

$18(35.3 \%)$

Facilitating Cue (FC) Condition

$(\mathrm{p}, \mathrm{FET}=.76, \mathrm{df}=1)$

Female

$16(80 \%)$

$13(17.8 \%)$

Male

$4(20 \%)$

Inhibiting Cue (FC) Condition

Female

Male

Note. Numbers in parentheses indicate row percentages. 
Table 15

Summary statistics of Participant Demographic Information for Study II and Study III(1)

\begin{tabular}{ccccc}
\hline & Monochromatic & $\begin{array}{c}\text { Facilitating Cue } \\
\text { Condition }\end{array}$ & $\begin{array}{c}\text { Inhibiting Cue } \\
\text { Condition }\end{array}$ \\
\hline \multirow{3}{*}{ Sample Size } & Female & $56(75.7 \%)$ & $45(88.2 \%)$ & $50(75.7 \%)$ \\
& Male & $18(24.3 \%)$ & $6(11.8 \%)$ & $16(24.3 \%)$ \\
& Total & 74 & 51 & 66 \\
\hline
\end{tabular}


Table 16

Descriptive Statistics of Participant Demographic Information for Study II and Study III(1)

\begin{tabular}{lcccc}
\hline & & Monochromatic & $\begin{array}{c}\text { Facilitating Cue } \\
\text { Condition }\end{array}$ & $\begin{array}{c}\text { Inhibiting Cue } \\
\text { Condition }\end{array}$ \\
\hline Age & M (SD) & $21.19(5.56)$ & $21.32(2.45)$ & $21.24(3.53)$ \\
SPA & M (SD) & $3.14(0.53)$ & $3.13(0.62)$ & $3.12(0.48)$ \\
General Anxiety & M (SD) & $34.68(13.34)$ & $32.62(11.25)$ & $34.50(13.77)$ \\
\hline
\end{tabular}


Table 17

Summary Statistics of Participant Demographic Information for Study III (2)

\begin{tabular}{ccccc}
\hline & & Monochromatic & $\begin{array}{c}\text { Facilitating Cue } \\
\text { Condition }\end{array}$ & $\begin{array}{c}\text { Inhibiting Cue } \\
\text { Condition }\end{array}$ \\
\hline \multirow{3}{*}{ Sample Size } & Female & $90(72.6 \%)$ & $64(80 \%)$ & $74(72.5 \%)$ \\
& Male & $34(27.4 \%)$ & $16(20 \%)$ & $28(27.5 \%)$ \\
& Total & 124 & 80 & 102 \\
\hline
\end{tabular}


Table 18

Correlation between Cognitive Strategy Flexibility and Mental rotation performance Change points

\begin{tabular}{cllc}
\cline { 2 - 4 } & \multirow{2}{*}{ Monochromatic } & Pearson Correlation & $0.46^{* *}$ \\
\cline { 2 - 4 } Mental Rotation & $\mathrm{N}$ & 121 \\
\cline { 2 - 4 } Score & Facilitating Cue & Pearson Correlation & $0.53^{* *}$ \\
& (FC) & $\mathrm{N}$ & 77 \\
\cline { 2 - 4 } & \multirow{2}{*}{ Inhibiting Cue (IC) } & Pearson Correlation & $0.56^{* *}$ \\
& $\mathrm{~N}$ & 99
\end{tabular}

**. Significant at $\mathrm{p}(2$-tailed $)<.00$

Control variable - spatial anxiety 
Table 19

Cognitive Strategy Flexibility by Participant Sex

\begin{tabular}{lccccc}
\hline \multirow{2}{*}{ MRT Score } & \multicolumn{2}{c}{ Male } & \multicolumn{2}{c}{ Female } & Significance \\
\cline { 2 - 6 } & $\mathrm{M}$ & $\mathrm{SD}$ & $\mathrm{M}$ & $\mathrm{SD}$ & $\mathrm{p}$ \\
\hline Monochromatic & 415.29 & 203.81 & 369.38 & 199.60 & $\mathrm{t}(122)=-1.14, \mathrm{p}=.26$ \\
Facilitating Cue (FC) & 428.13 & 217.09 & 383.80 & 170.74 & $\mathrm{t}(78)=-0.88, \mathrm{p}=.38$ \\
Inhibiting Cue (IC) & 422.43 & 260.26 & 404.31 & 224.01 & $\mathrm{t}(100)=-0.33, \mathrm{p}=.73$ \\
\hline$* \mathrm{p}<.05$ & & & & &
\end{tabular}


Table 20

Correlation between Cognitive Strategy Flexibility and Early Spatial Activity Experience

\begin{tabular}{|c|c|c|c|c|}
\hline & $\begin{array}{l}\text { Spatial } \\
\text { Activities }\end{array}$ & $\begin{array}{l}\text { Male Sex- } \\
\text { typed } \\
\text { Spatial } \\
\text { Activities }\end{array}$ & $\begin{array}{l}\text { Female Sex- } \\
\text { typed Spatial } \\
\text { Activities }\end{array}$ & $\begin{array}{l}\text { Neutral } \\
\text { Spatial } \\
\text { Activities }\end{array}$ \\
\hline \multicolumn{5}{|l|}{ Monochromatic Condition } \\
\hline Pearson’s Correlation & $.31 *$ & $.24^{*}$ & $.35 * *$ & .20 \\
\hline Significance (2-tailed) & .01 & .04 & .00 & .09 \\
\hline Degree of Freedom & 69 & 69 & 69 & 69 \\
\hline \multicolumn{5}{|l|}{ Facilitating cue (FC) } \\
\hline \multicolumn{5}{|l|}{ Condition } \\
\hline Pearson's Correlation & -.06 & -.06 & .03 & -.10 \\
\hline Significance (2-tailed) & .71 & .71 & .86 & .53 \\
\hline Degree of Freedom & 41 & 41 & 41 & 41 \\
\hline \multicolumn{5}{|l|}{ Inhibiting cue (IC) } \\
\hline \multicolumn{5}{|l|}{ Condition } \\
\hline Pearson's Correlation & .10 & .01 & .17 & .10 \\
\hline Significance (2-tailed) & .46 & .94 & .20 & .46 \\
\hline Degree of Freedom & 53 & 53 & 53 & 53 \\
\hline
\end{tabular}

*. Significant at $\mathrm{p}(2$-tailed $)<.05$

$* *$. Significant at $\mathrm{p}(2$-tailed $)<.001$

Control variable - spatial anxiety 


\section{FIGURES}

Figure 1. A sample stimulus used in the monochromatic experimental condition, based on the Shepard and Metzler (1971) mental rotation task. The two figures are similar and can be rotated into congruence. The correct participant response for this stimulus is 'match'.

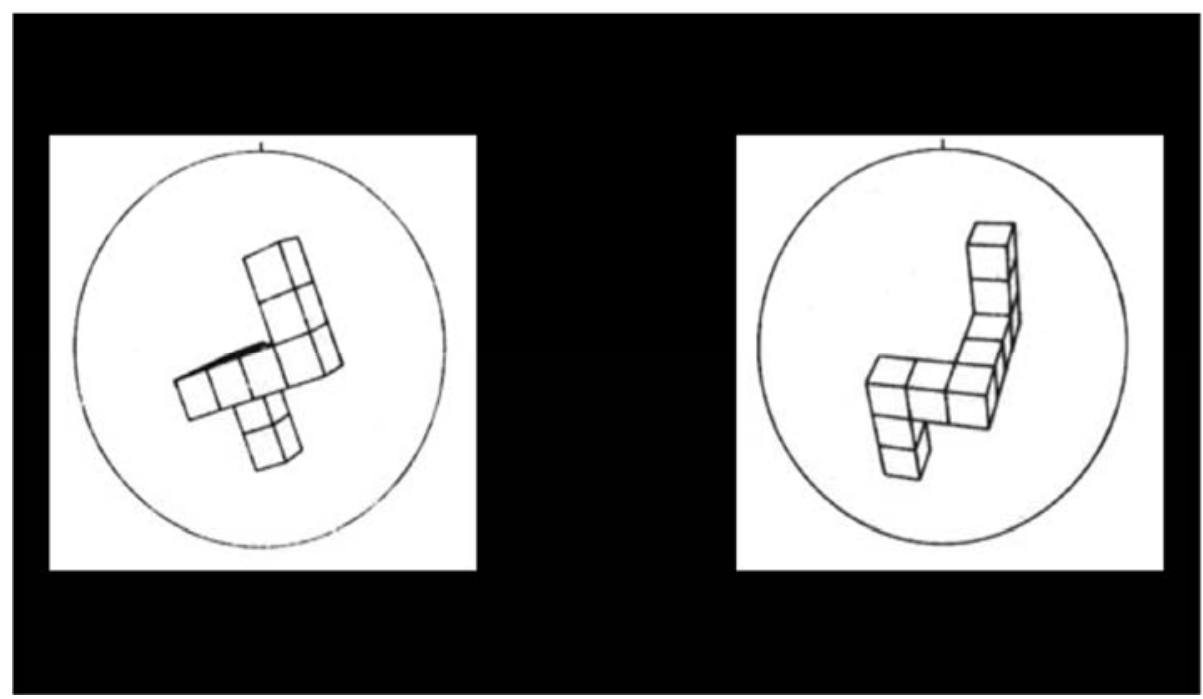


Figure 2. A sample stimulus used in the monochromatic experimental condition, based on the Shepard and Metzler (1971) mental rotation task. The two figures are mirror images of each other and cannot be rotated into congruence. The correct participant response for this stimulus is 'non-match'.

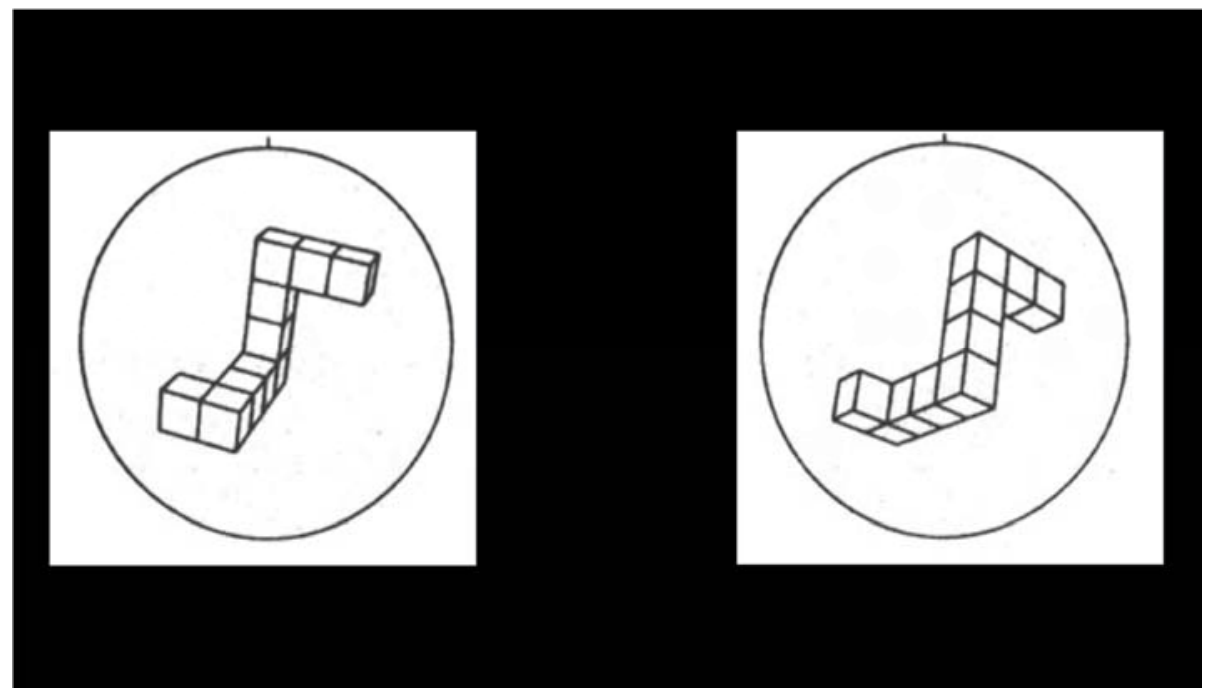


Figure 3: Overarching research question guiding the current dissertation: Do experiential factors affect adult mental rotation performance through the development of an optimum cognitive strategy?

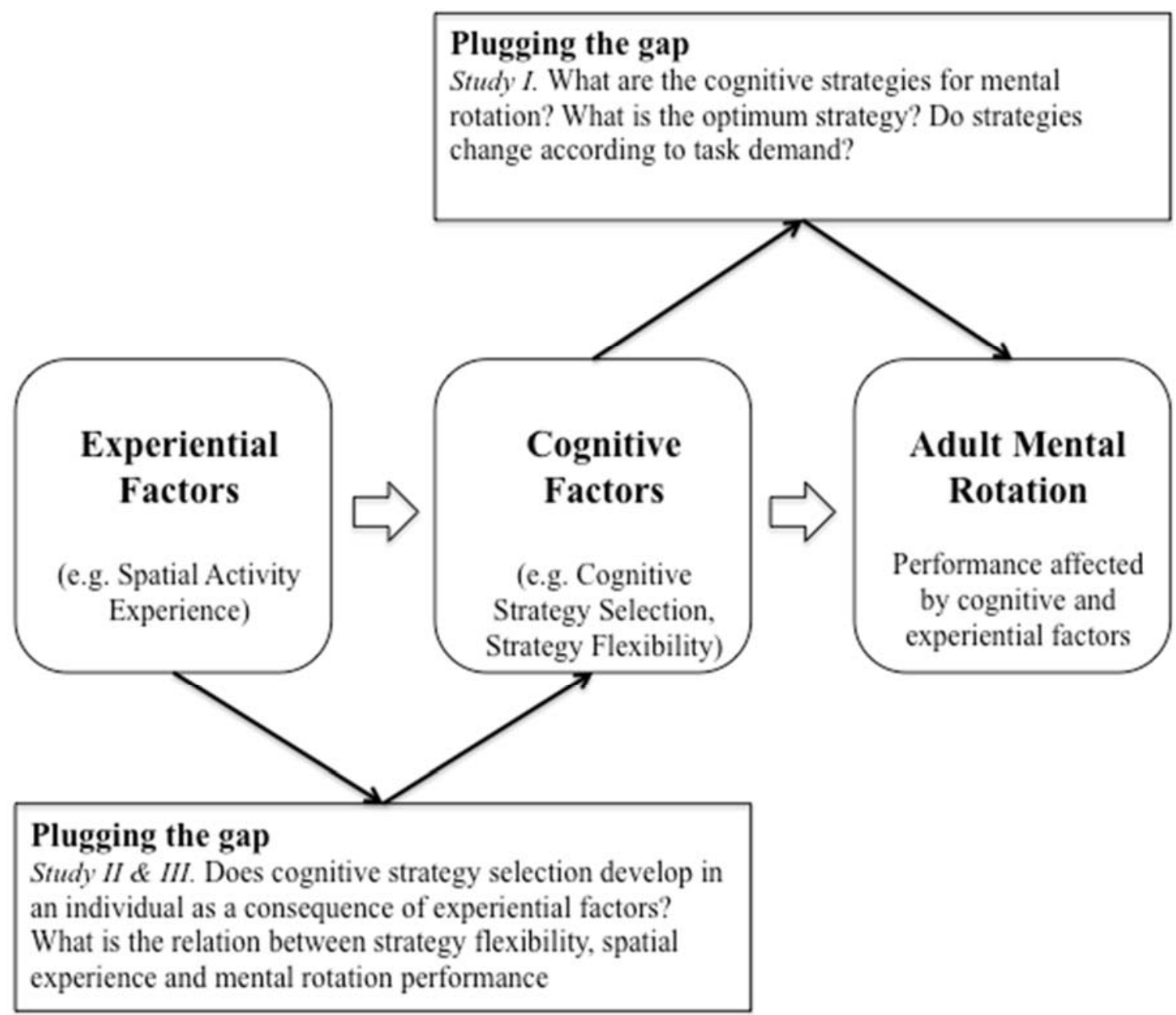


Figure 4. Hypothesized eye-gaze pattern of a piecemeal strategy with a longer confirmation stage, characterized by frequent peaks.

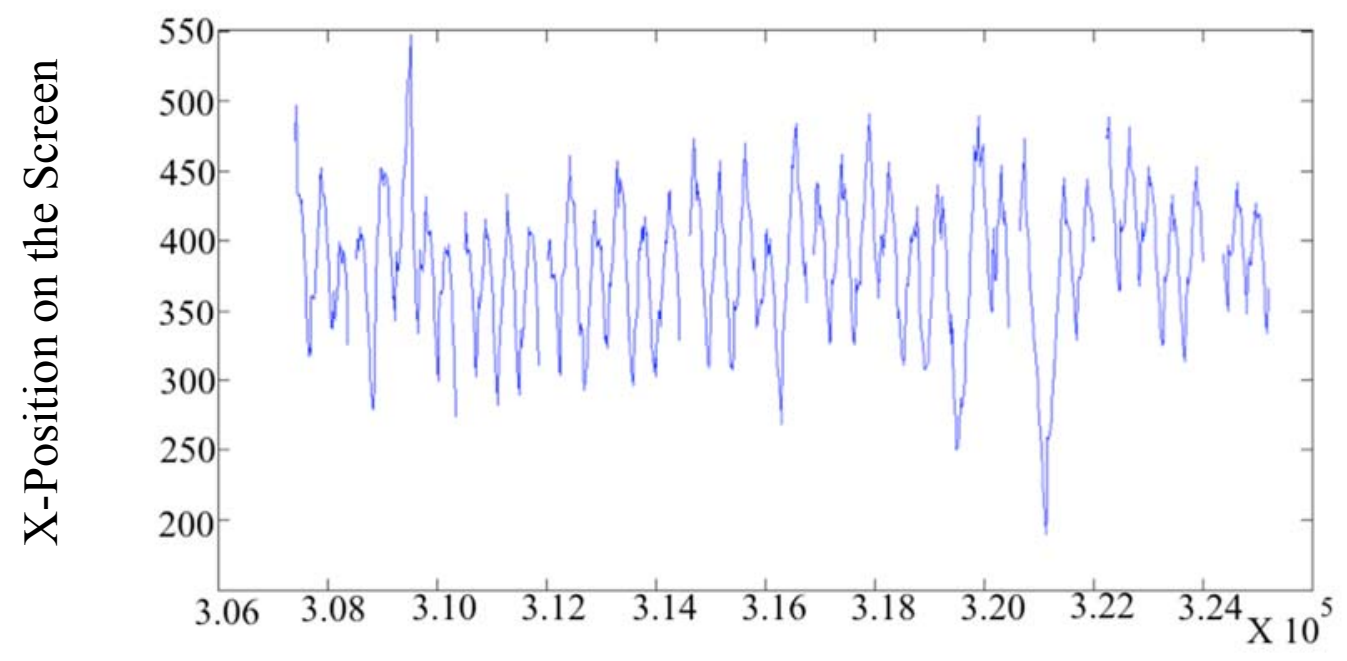

Time in Milliseconds 
Figure 5. Hypothesized eye-gaze pattern of a holistic strategy with a longer transformation and comparison stage, characterized by frequent plateaus.

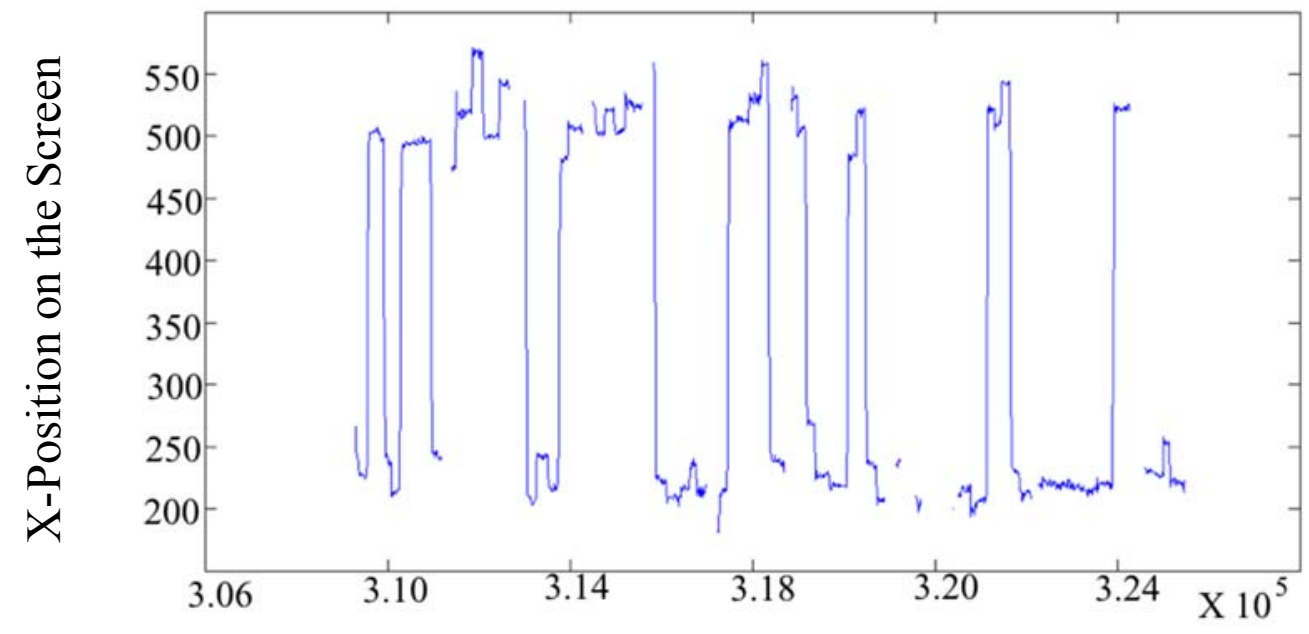

Time in Milliseconds 
Figure 6. A sample stimulus used in the facilitating cue (FC) condition. The red cube in the left figure corresponds to the red cube in the right figure. Therefore, the non-spatial color information is hypothesized to facilitate mental rotation.

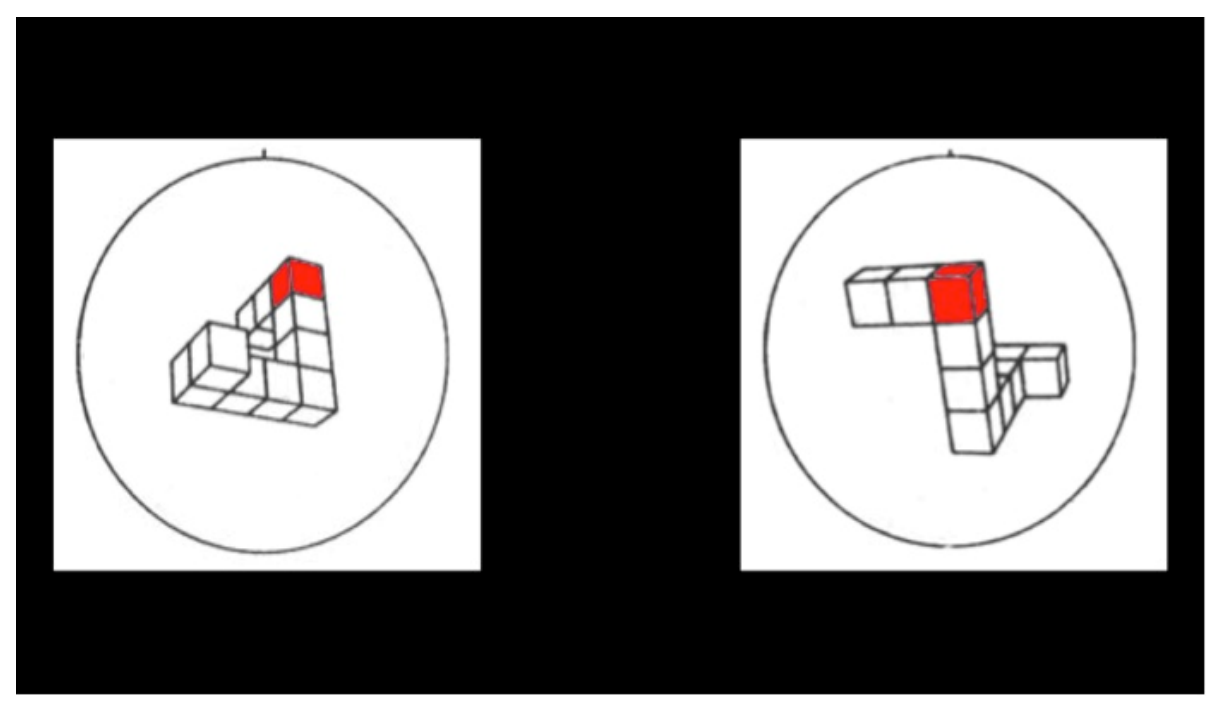


Figure 7. A sample stimulus used in the inhibiting cue (IC) condition. The red cube in the left figure does not correspond to the red cube in the right figure. Therefore, the nonspatial color information is hypothesized to inhibit mental rotation.

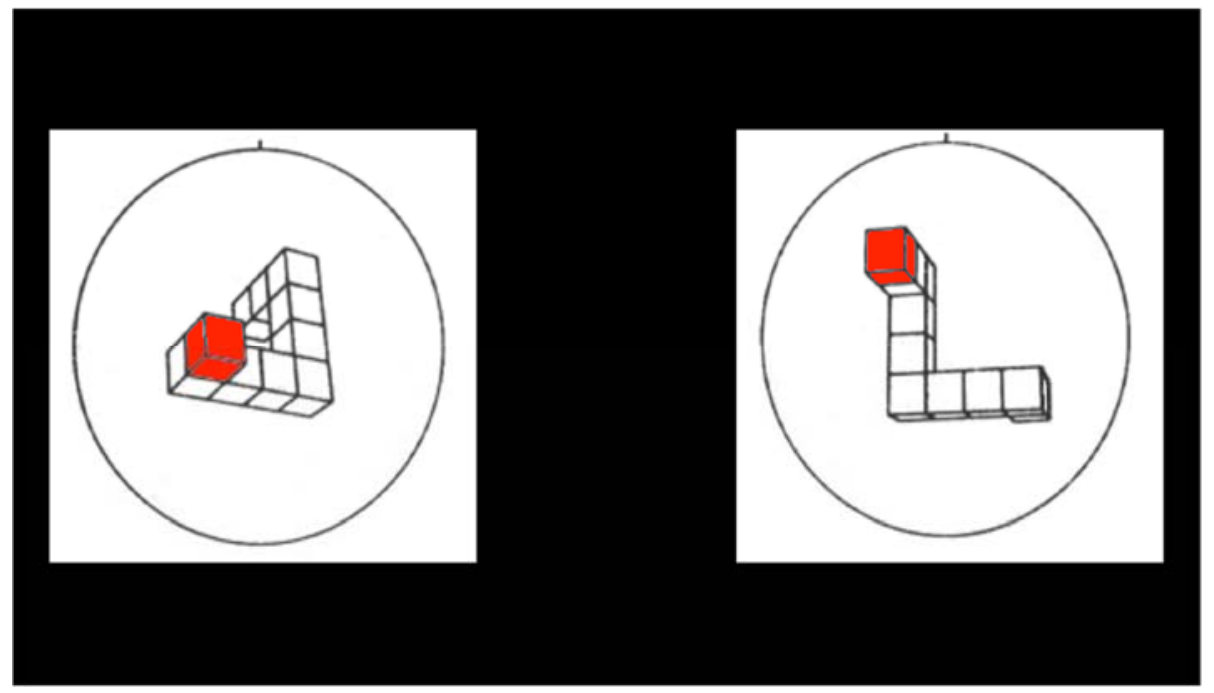


Figure 8. Flowchart of procedure and measures used in the current dissertation

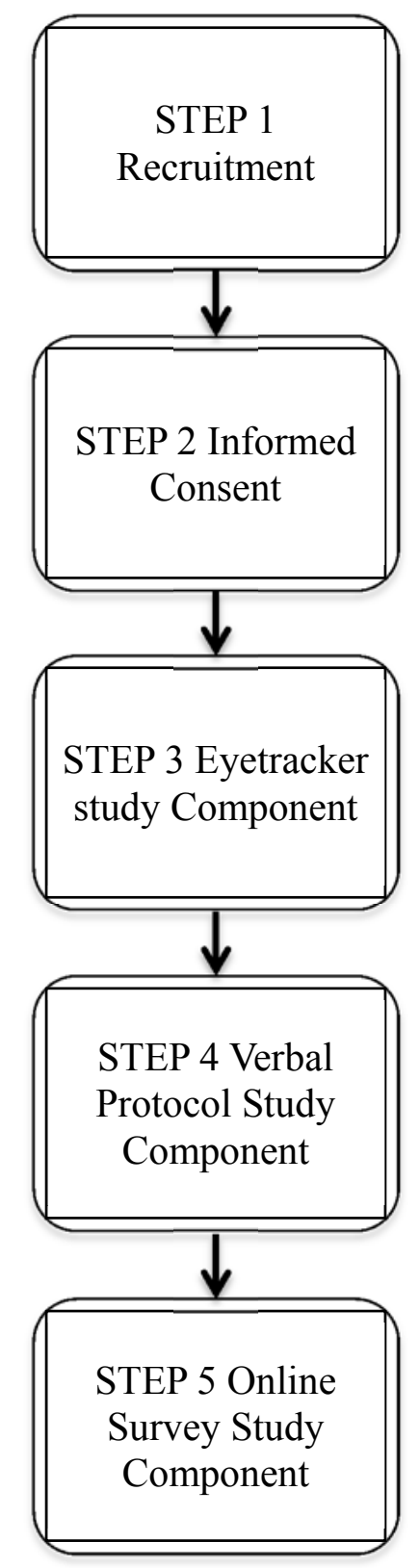

1. SONA SYSTEM - online recruitment system

2. Eligibility Criteria - Age between 18 and 25 years

1. Signed adult consent preapproved by IRB

2. Participation was voluntary and participants could withdraw any time during the study

1. Randomly assigned to one of three experimental conditions - Monochromatic, Facilitating Cue (FC) or Inhibiting Cue (IC) Conditions

2. Stimuli within each experimental condition was presented in two ways to counterbalance for fatigue

1. Asked a series of questions related to the task completed

2. Questions were asked in a fixed format

1. Filled out an online survey which presented measures in a fixed sequence 
Figure 9. Eye-tracking experimental room set-up

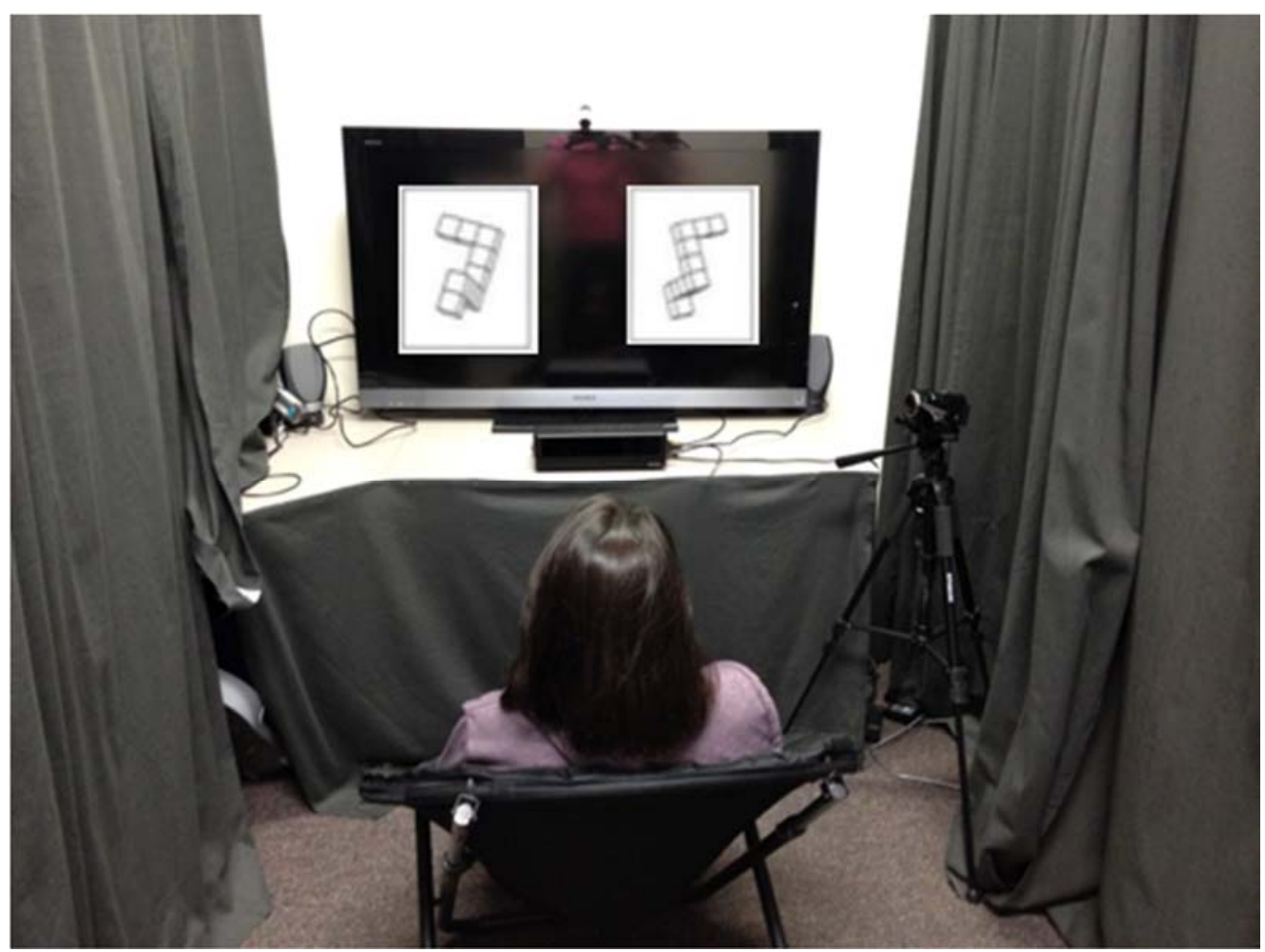


Figure 10. A sample stimulus used in the monochromatic condition. The red tinted box encompassing the figure indicates the area-of-interest (AOI) used to determine eye movement parameters.

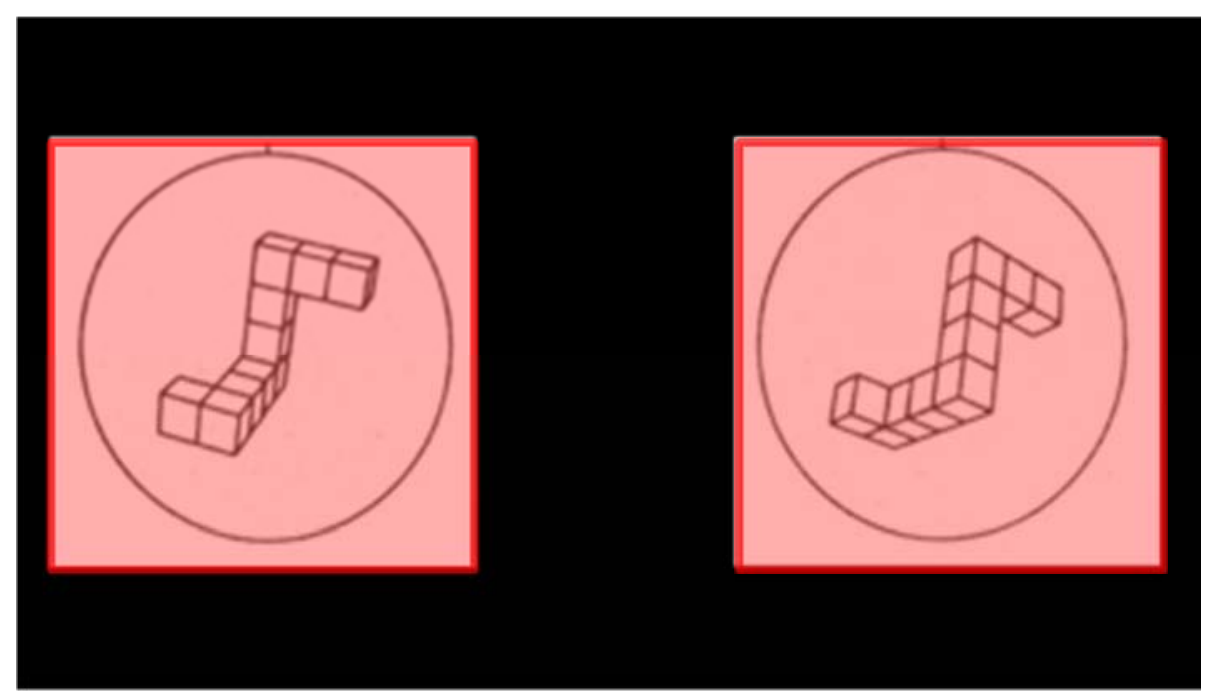


Figure 11. A sample stimulus used in the non-spatial cue condition. The red tinted box encompassing the red cube indicates the area-of-interest (AOI) used to determine eye movement parameters.

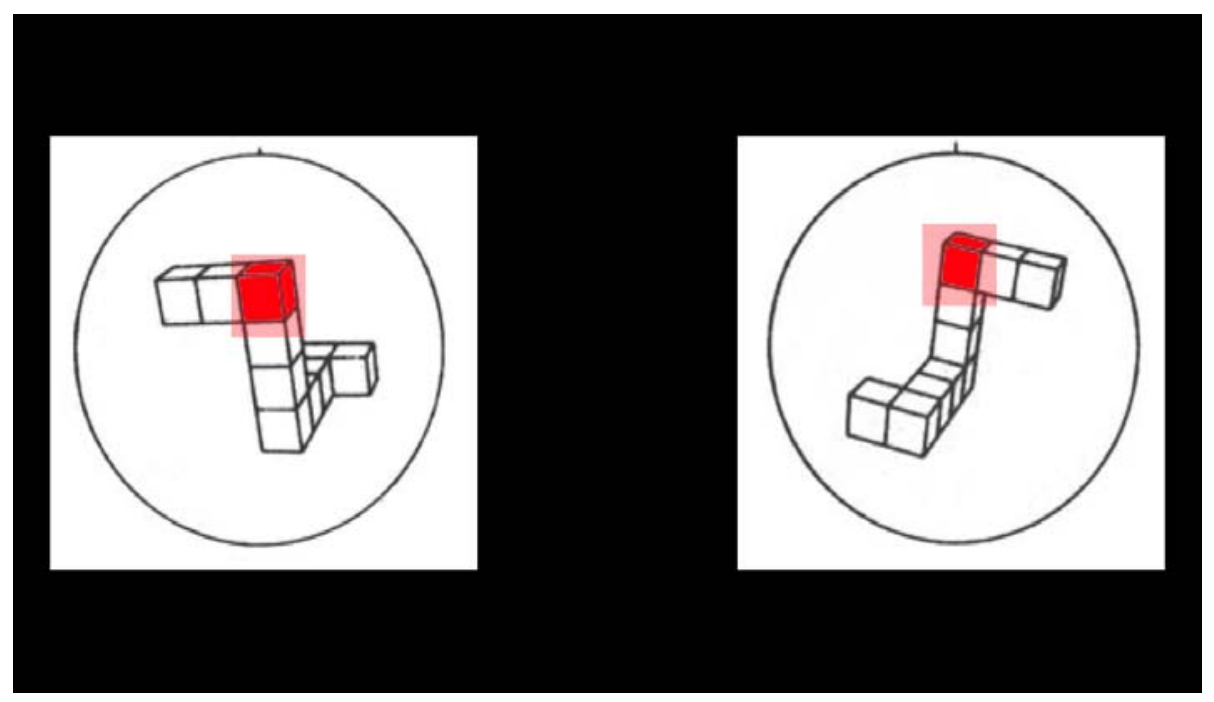


Figure 12. Relation between mental rotation score and experimental condition (by participant sex)

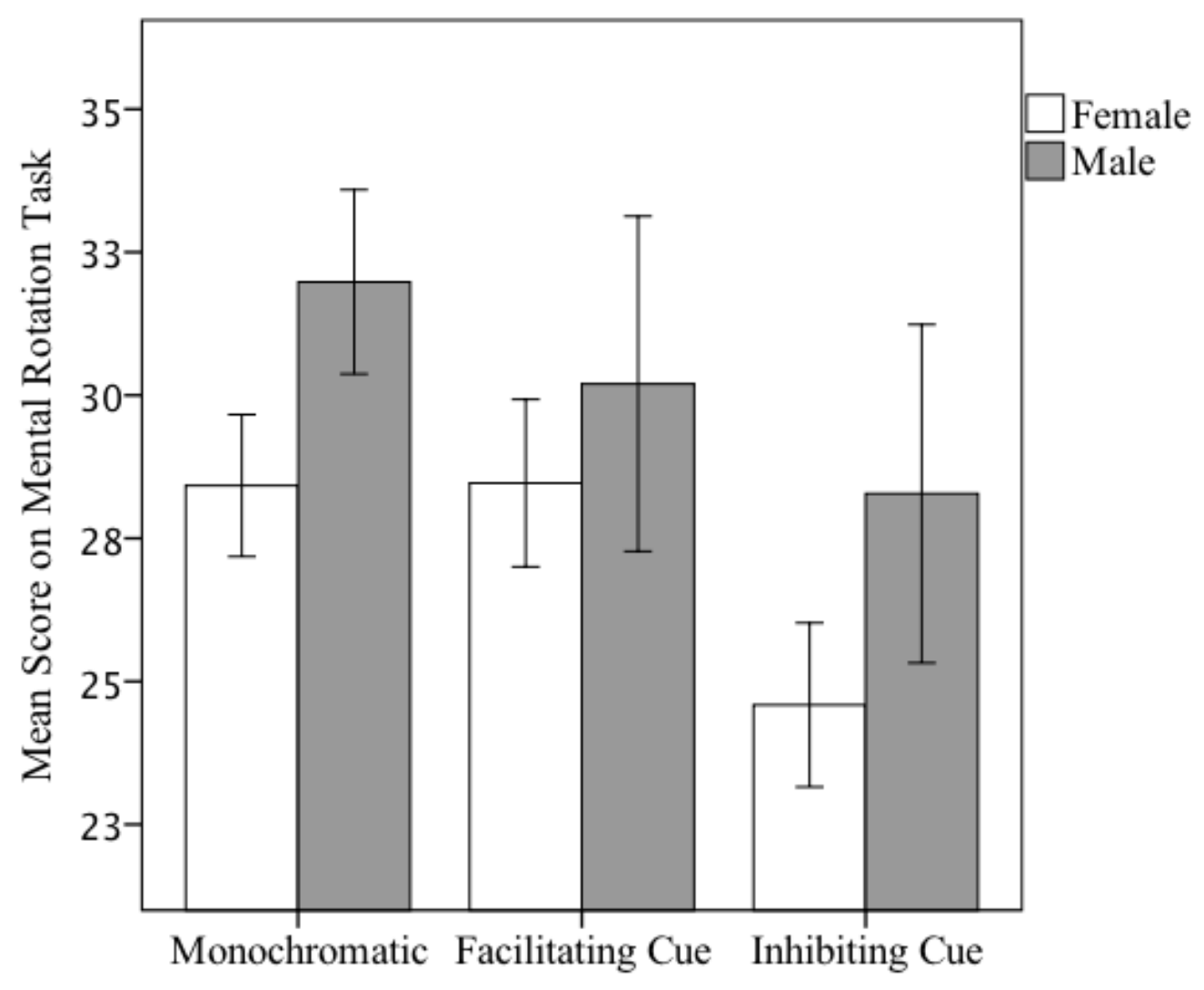


Figure 13. Relation between self-reported (verbal protocol) cognitive strategy selection and mental rotation performance

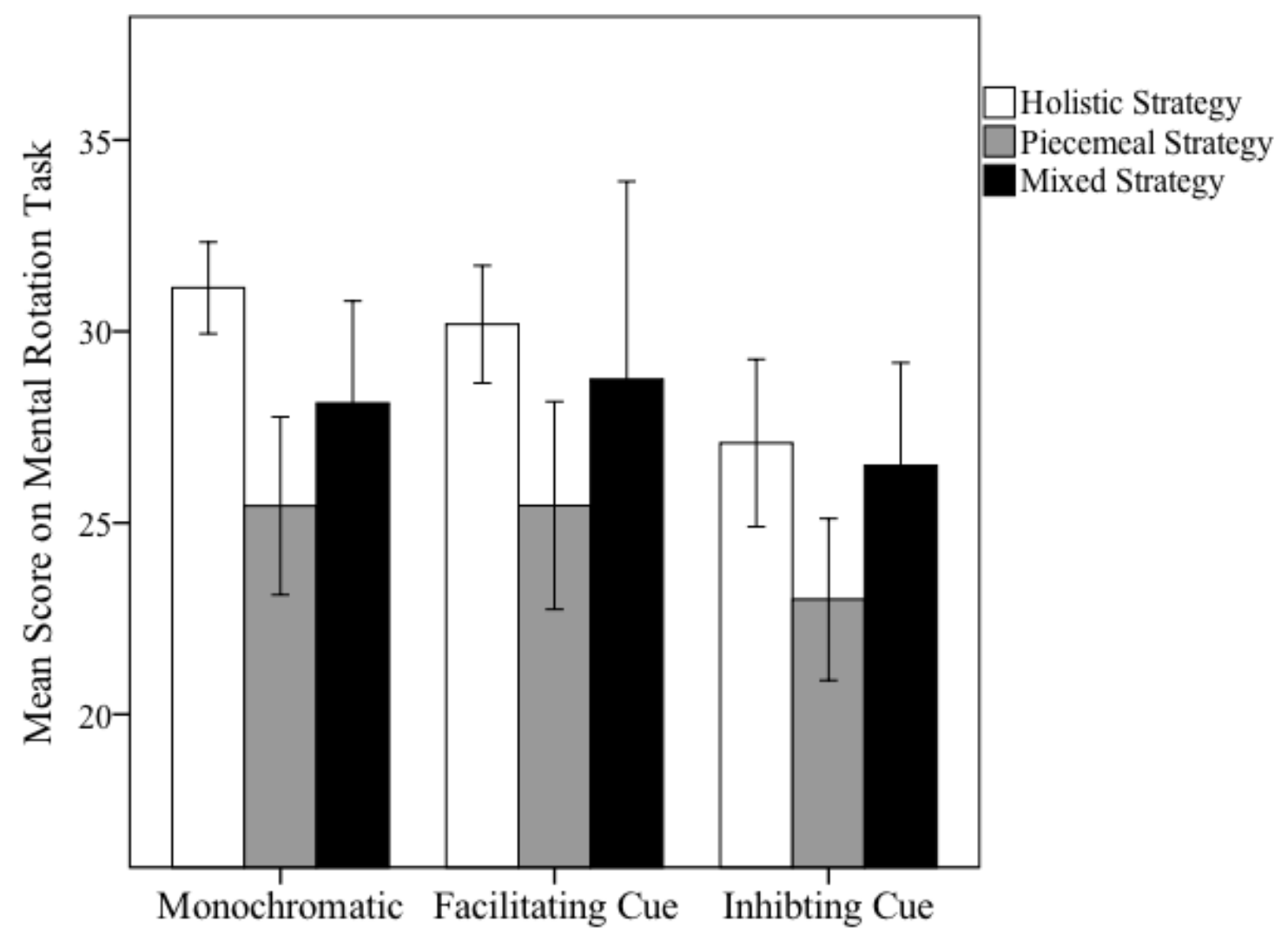


Figure 14. Relation between self-reported (verbal protocol) cognitive strategy selection and mental rotation performance for female participants only.

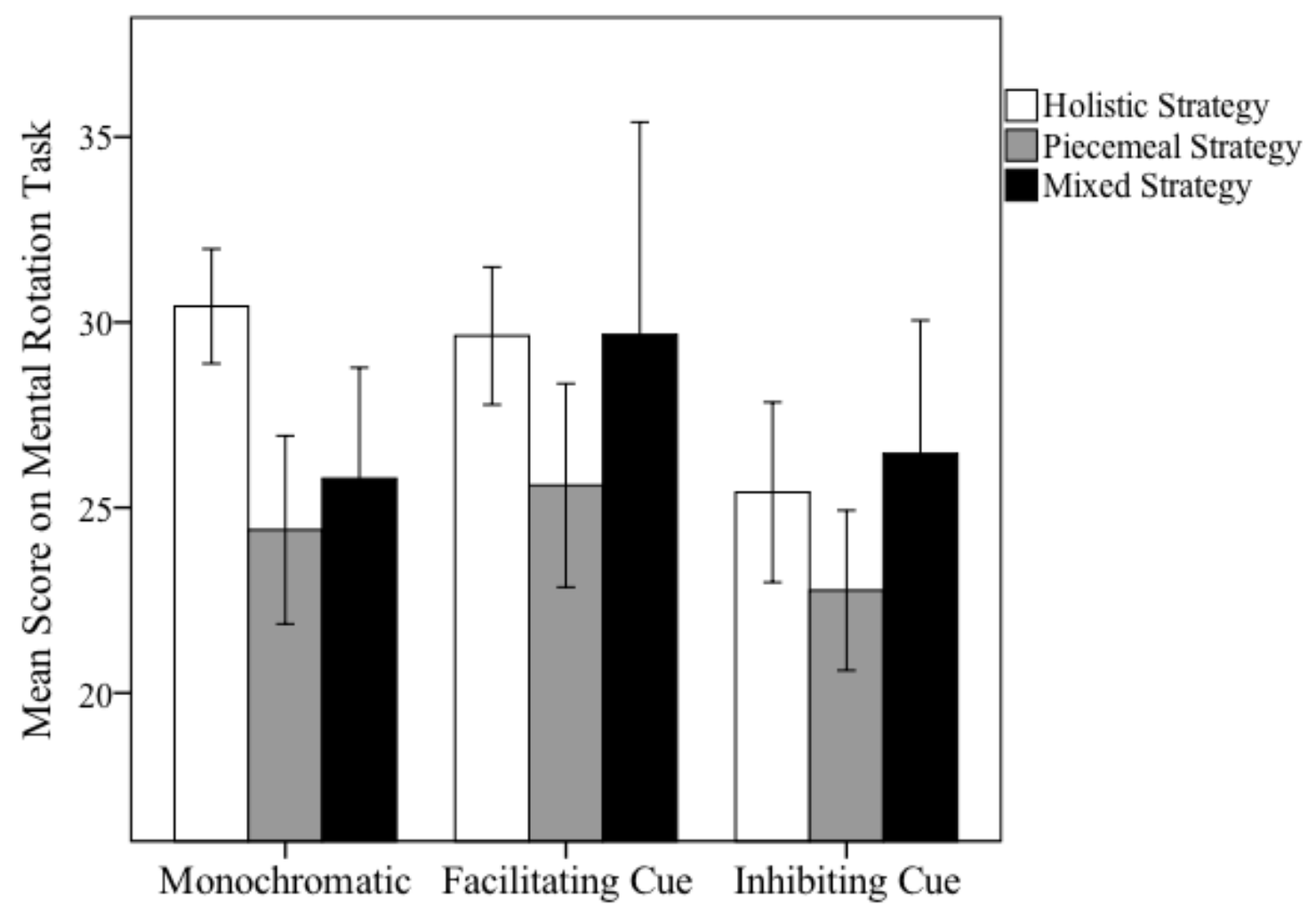


Figure 15. Relation between self-reported (verbal protocol) cognitive strategy selection and mental rotation performance for male participants only

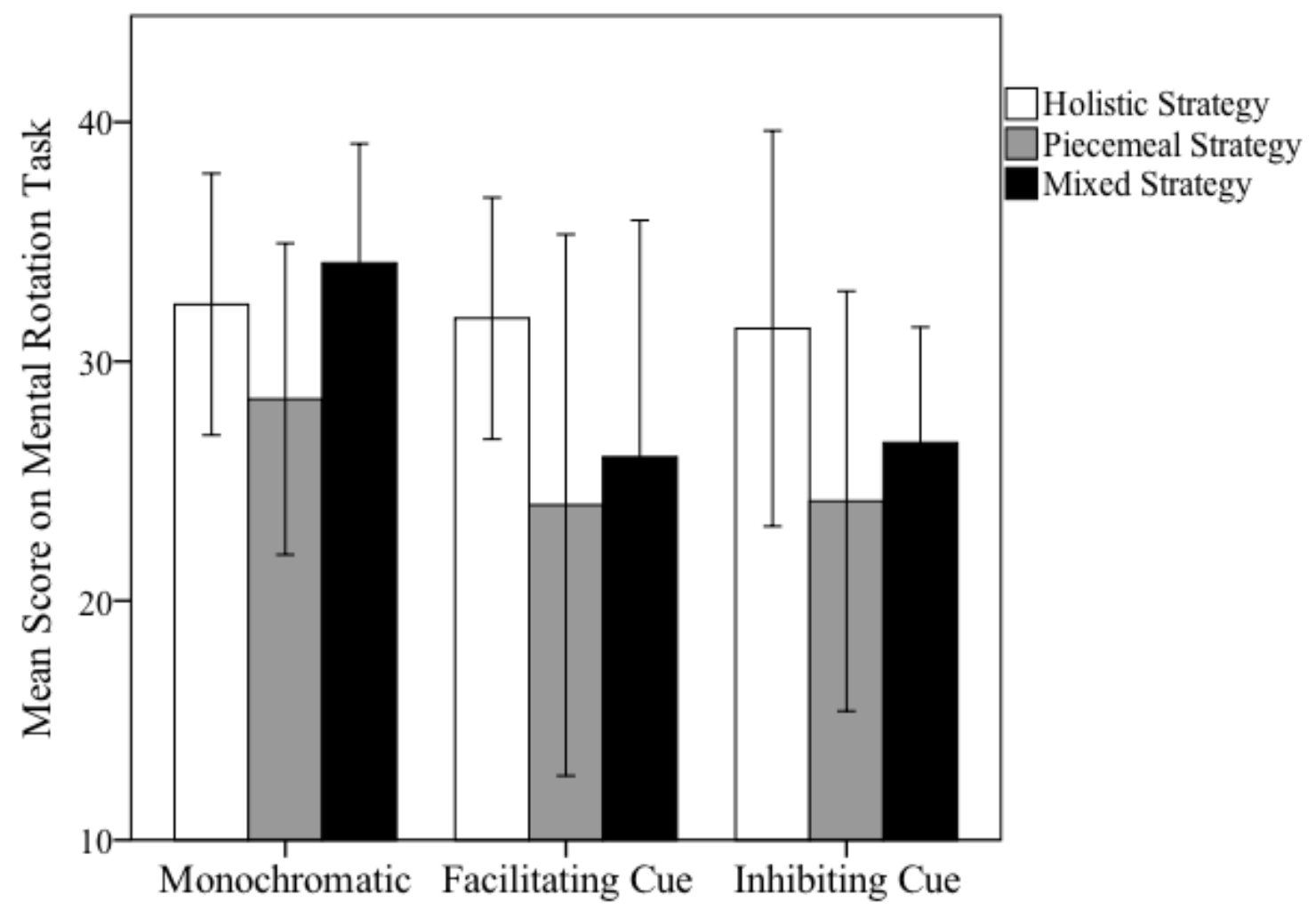


Figure 16. Relation between self-rated strategy items, calculated as a proportion of holistic to piecemeal item ratings, and experimental condition (by participant sex)

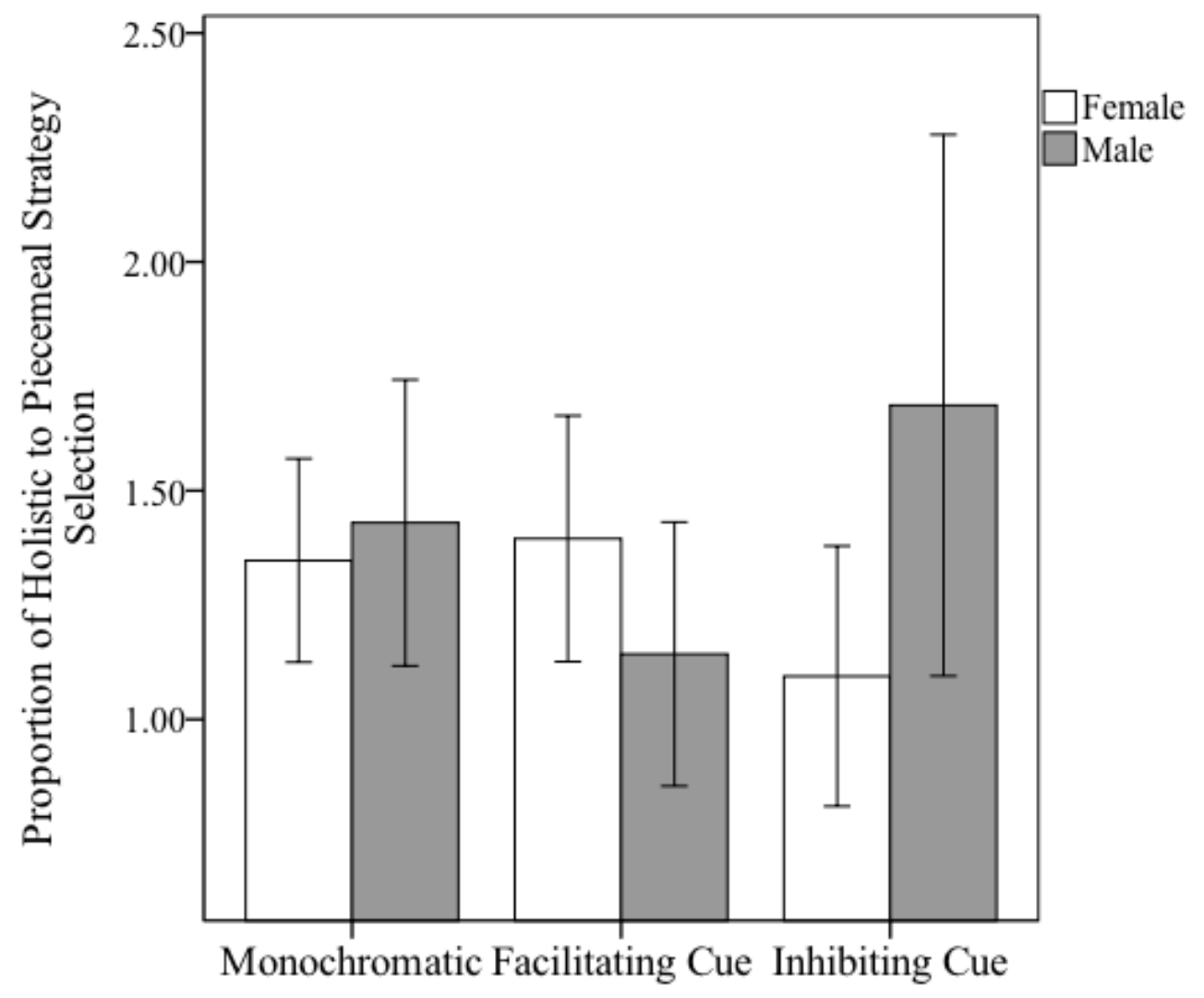


Figure 17. Eye movement in the monochromatic condition depicting eye gaze path (indicated by numbers) and duration (indicated by size).

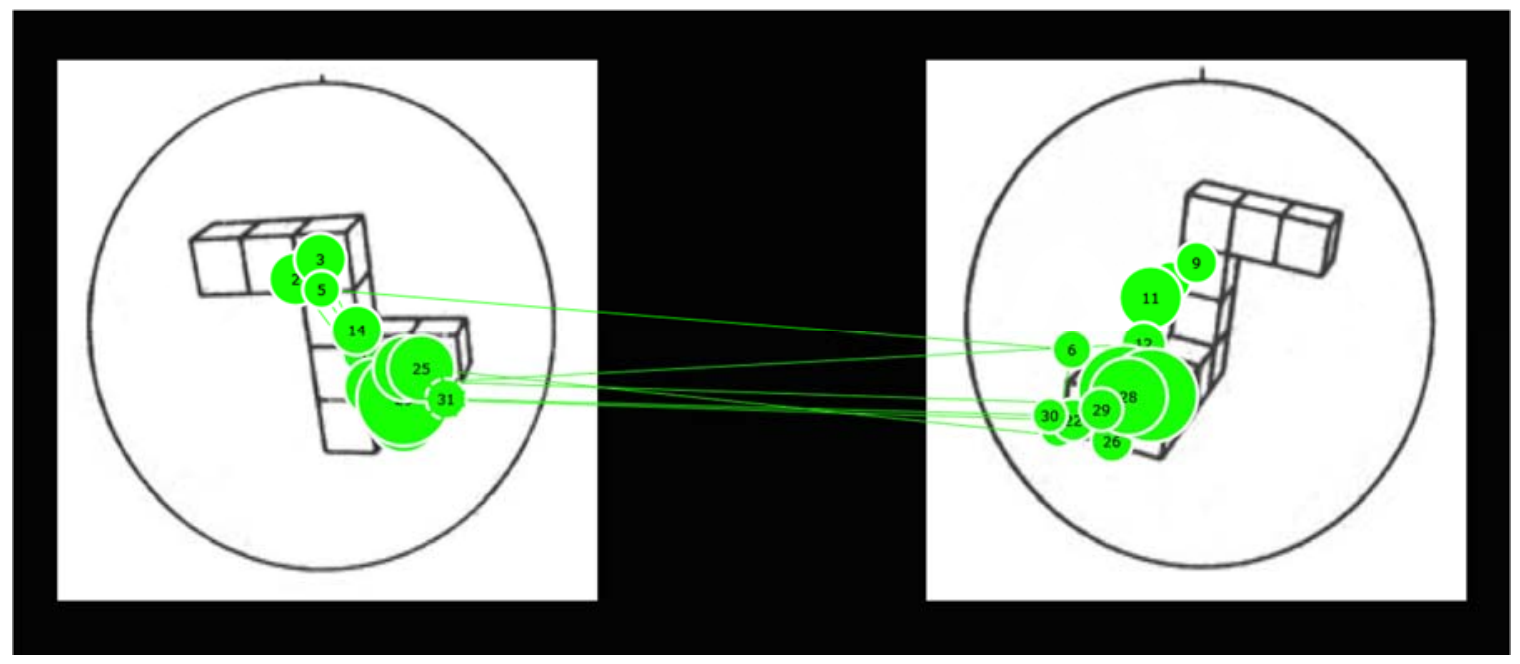


Figure 18. Eye movement in the facilitating cue (FC) condition depicting the use of the facilitating non-spatial information i.e. the red cube to enhance mental rotation

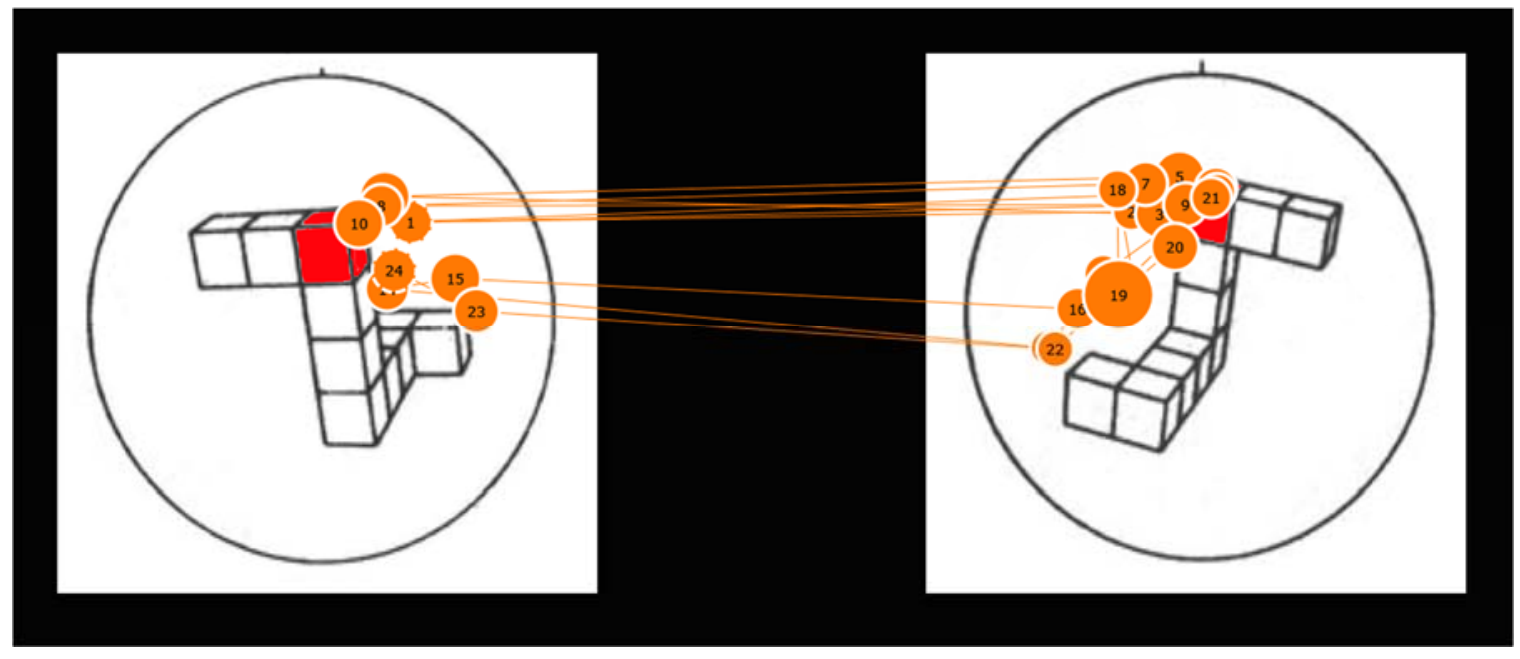


Figure 19. Eye movement in the inhibiting cue (IC) condition depicting the use of the inhibiting non-spatial information i.e. the red cube to inhibit mental rotation

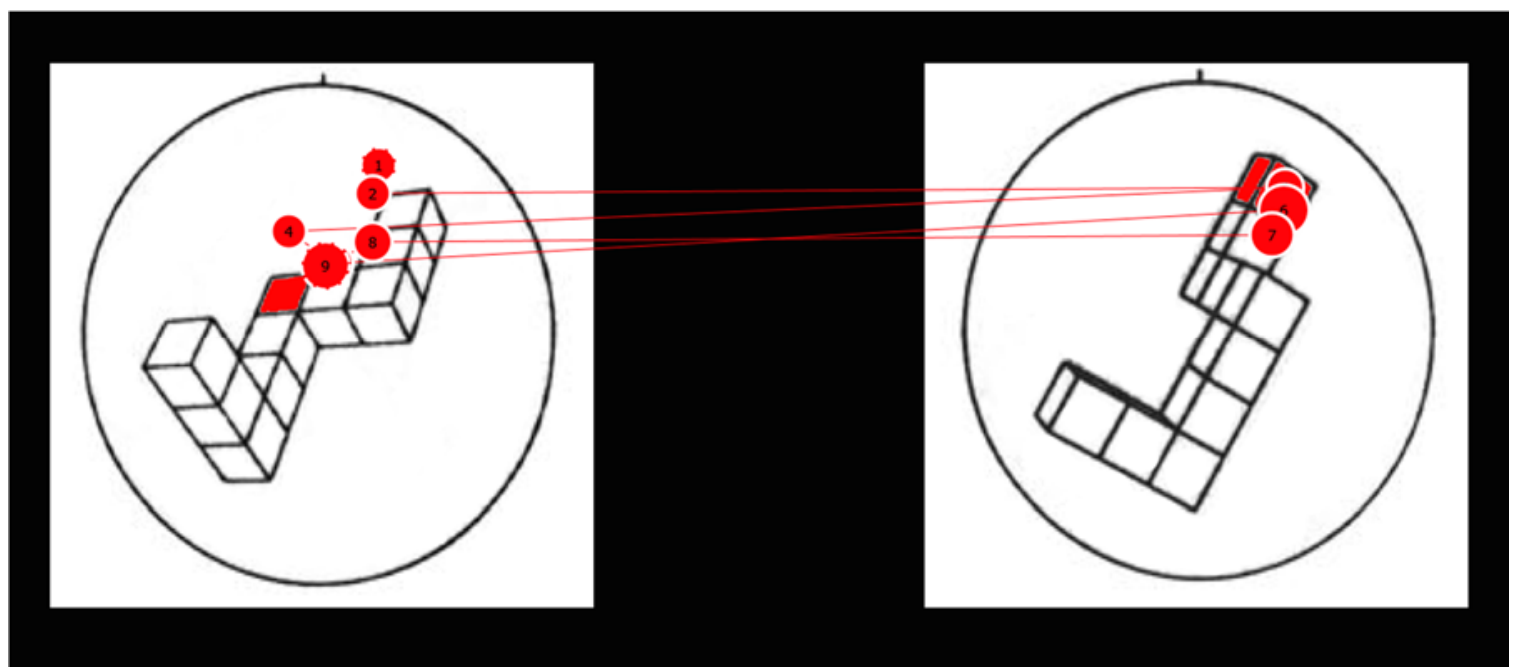


Figure 20. A 2-class latent profile model using mental rotation score (MRT) as a latent class predictor in the monochromatic condition

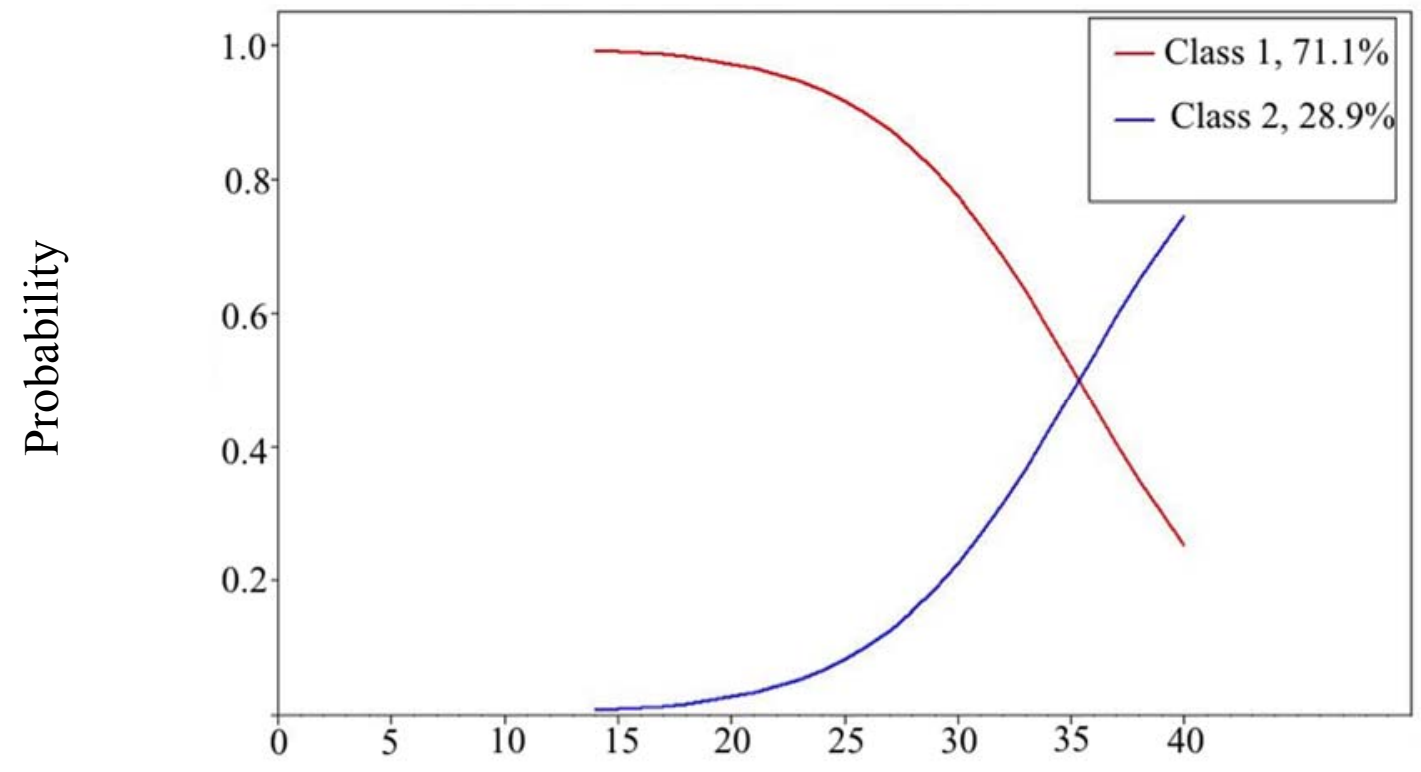

Mental Rotation Score 
Figure 21. A 2-class latent profile model using mental rotation score (MRT) as a latent class predictor in the facilitating cue (FC) condition

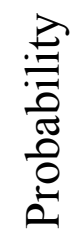

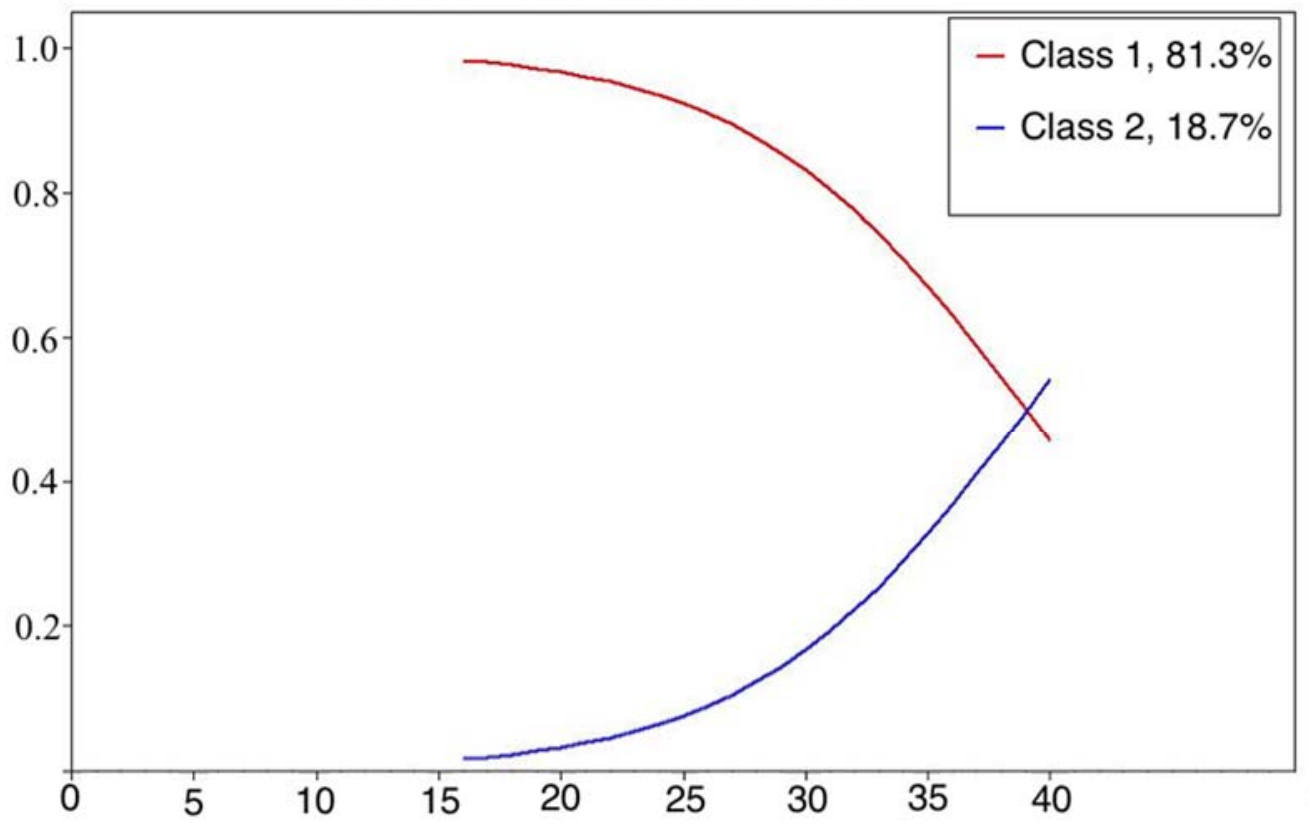

Mental Rotation Score 
Figure 22. Relation between latent classes and mental rotation performance in monochromatic condition (by participant sex).

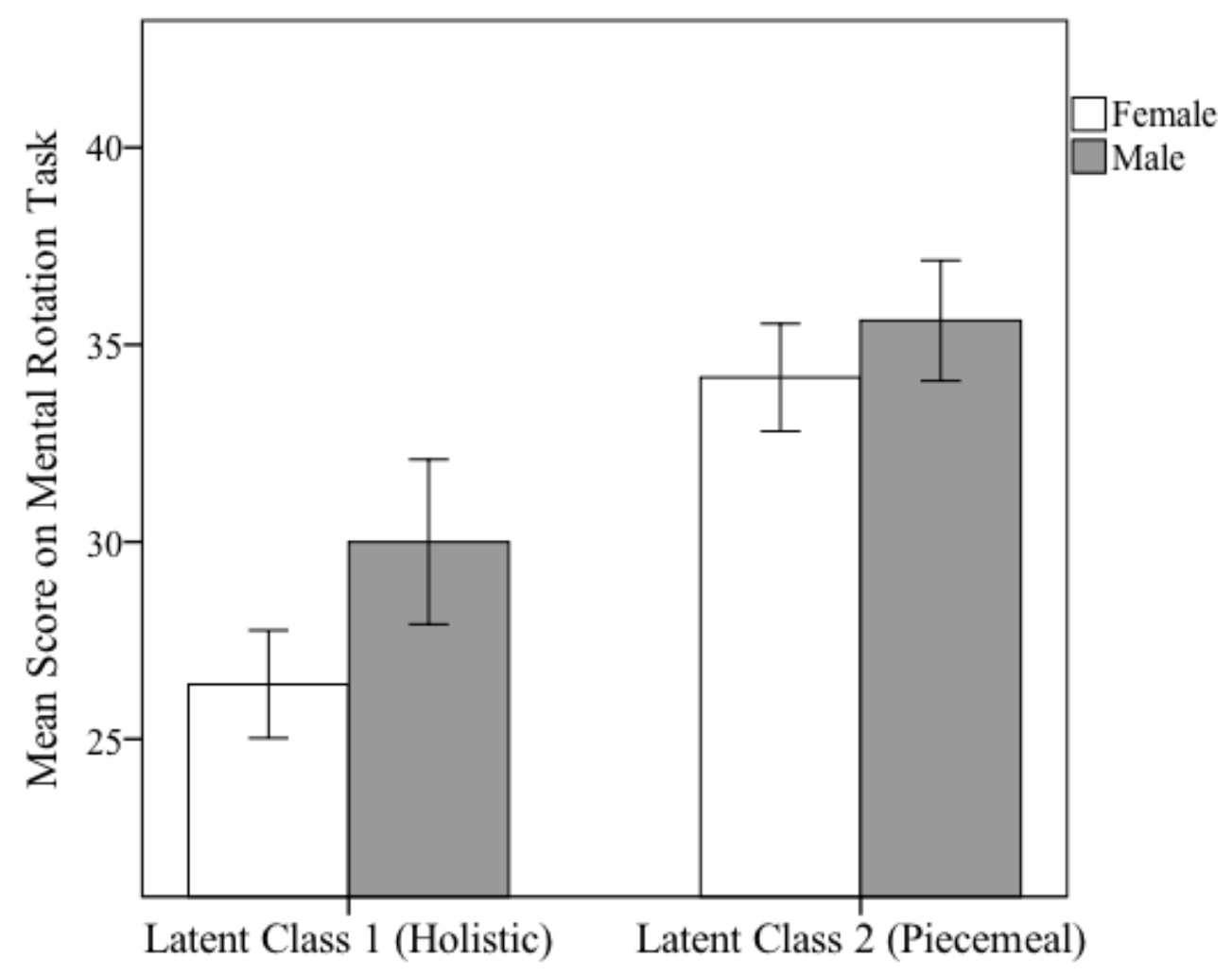


Figure 23. Relation between latent classes and mental rotation performance in facilitating cue condition (by participant sex).

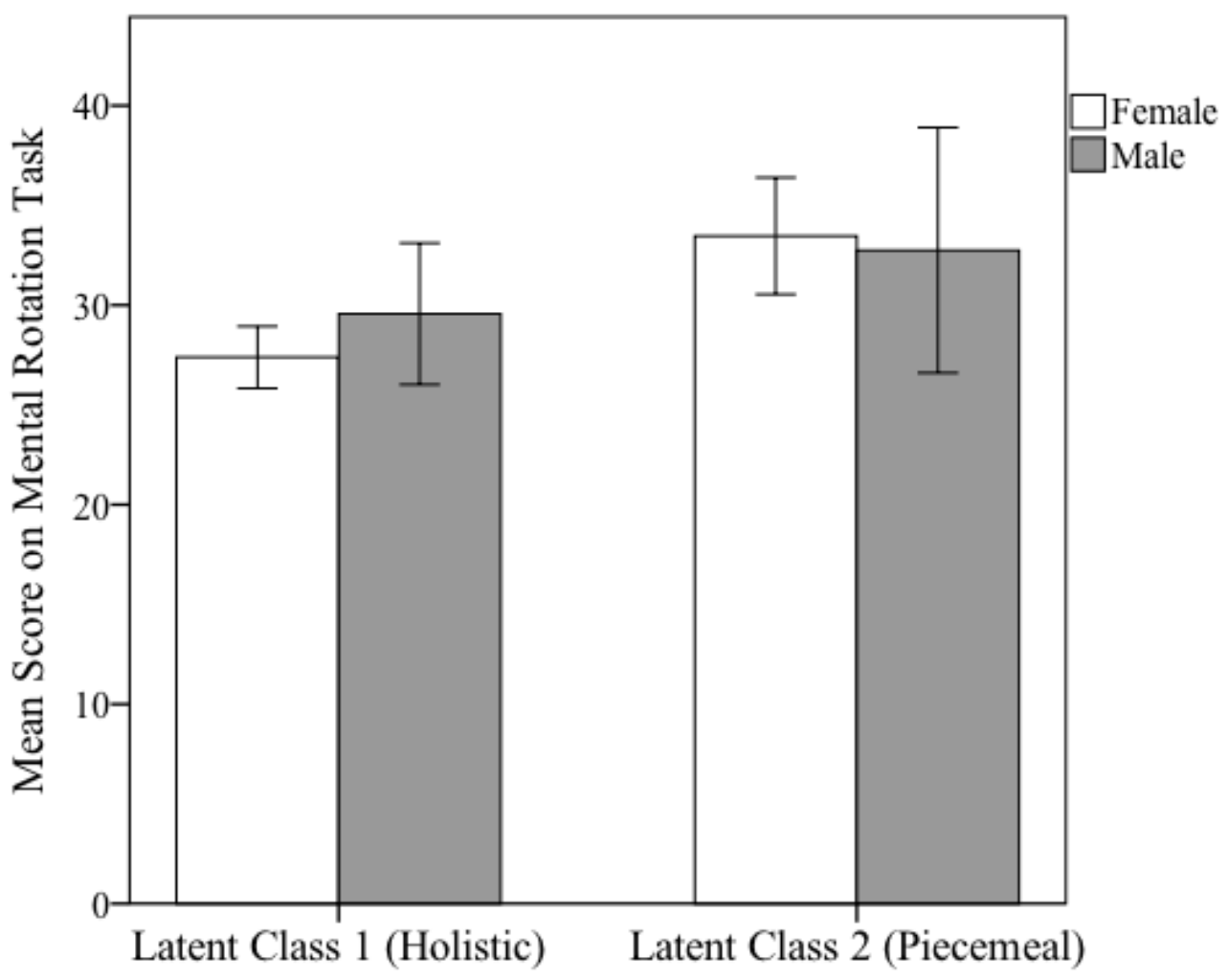


Figure 24. Sex differences in participation in male sex-typed spatial activity experience (by experimental condition)

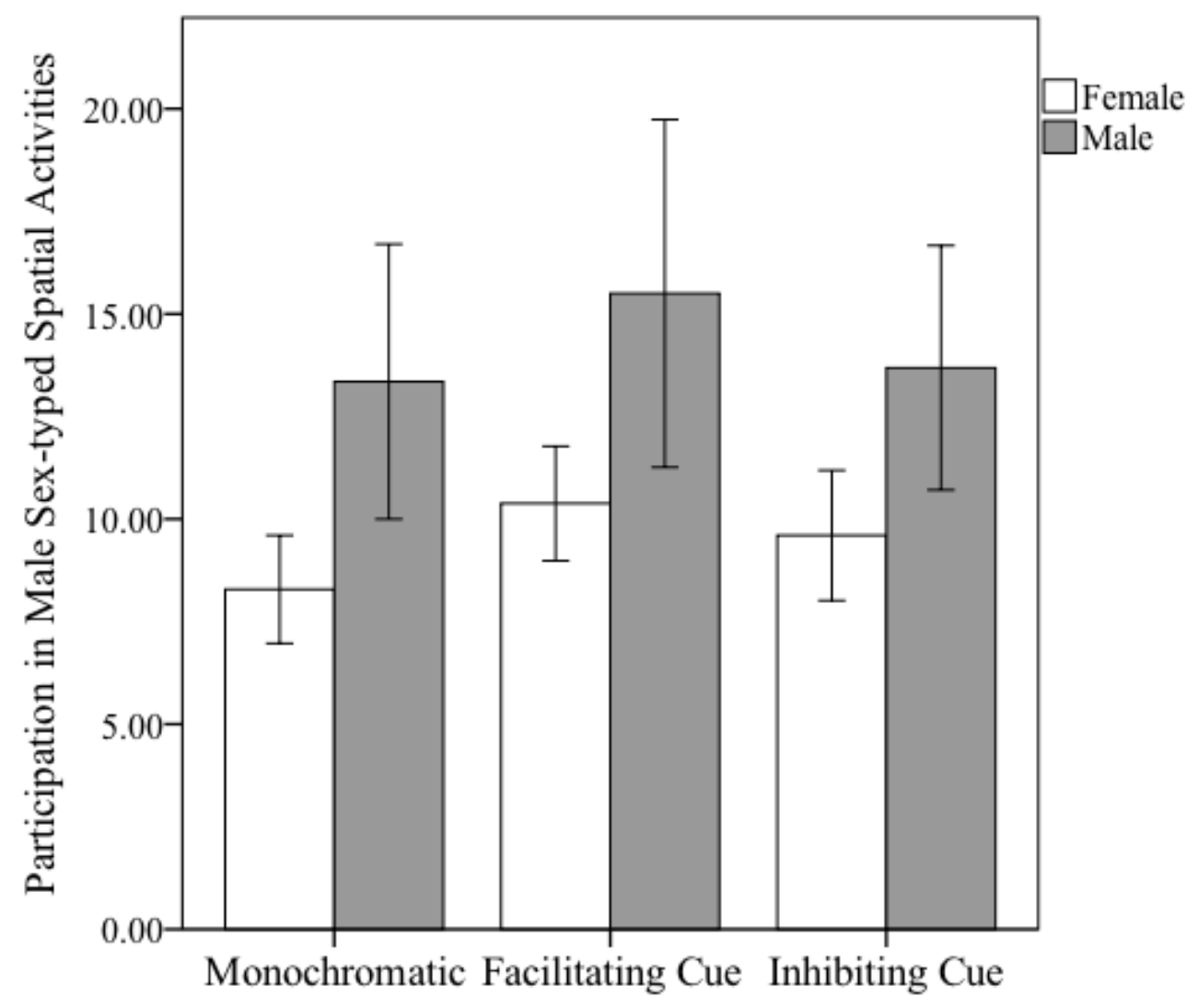


Figure 25. Sex differences in participation in female sex-typed spatial activity experience (by experimental condition)

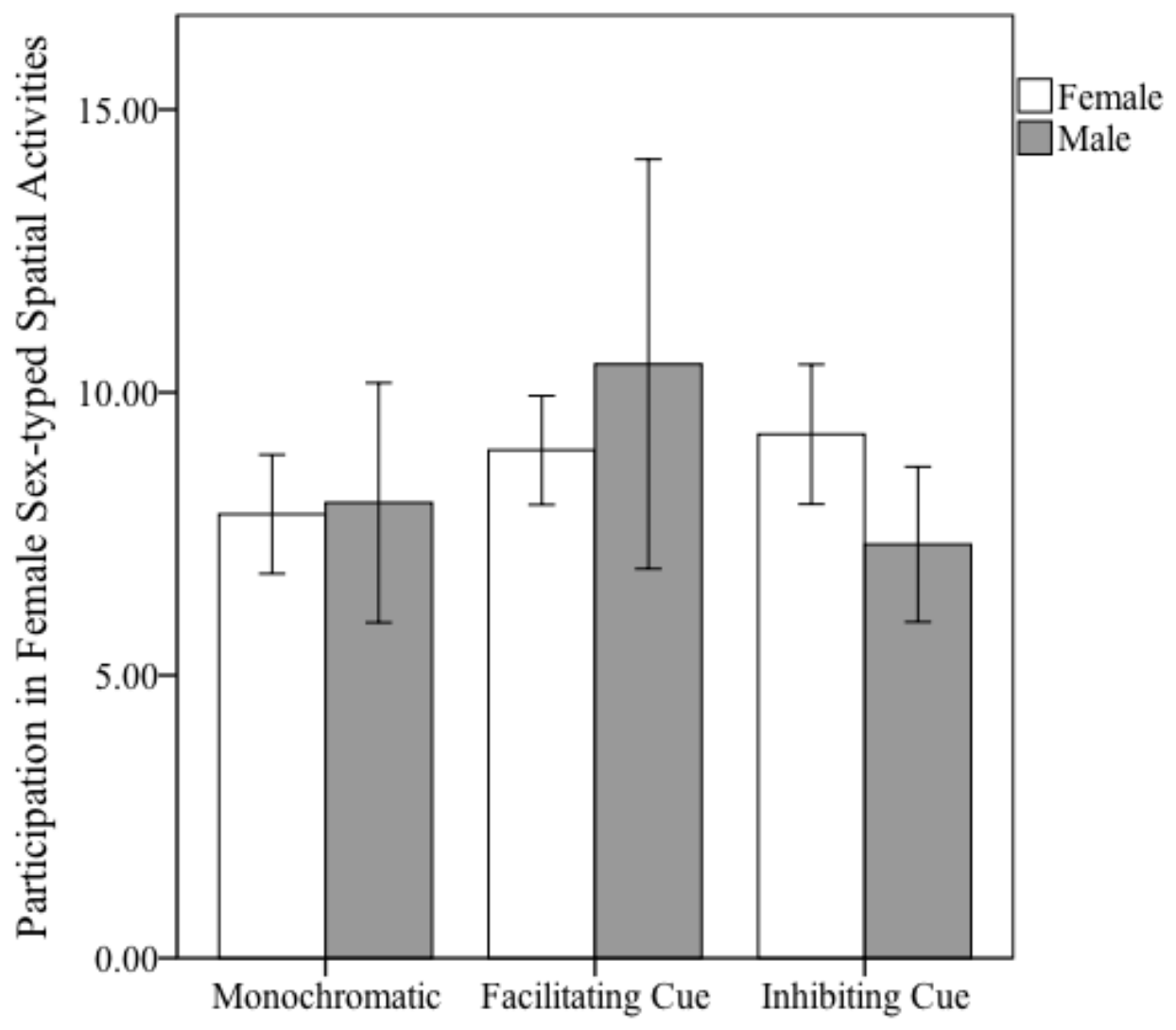


Figure 26. Scatterplot showing the relation between number of masculine spatial activities and MRT scores (top, ${ }^{\circ}$ males, ${ }^{+}$females; $r=0.31, p<0.05$ ), after controlling for "other" spatial activities.

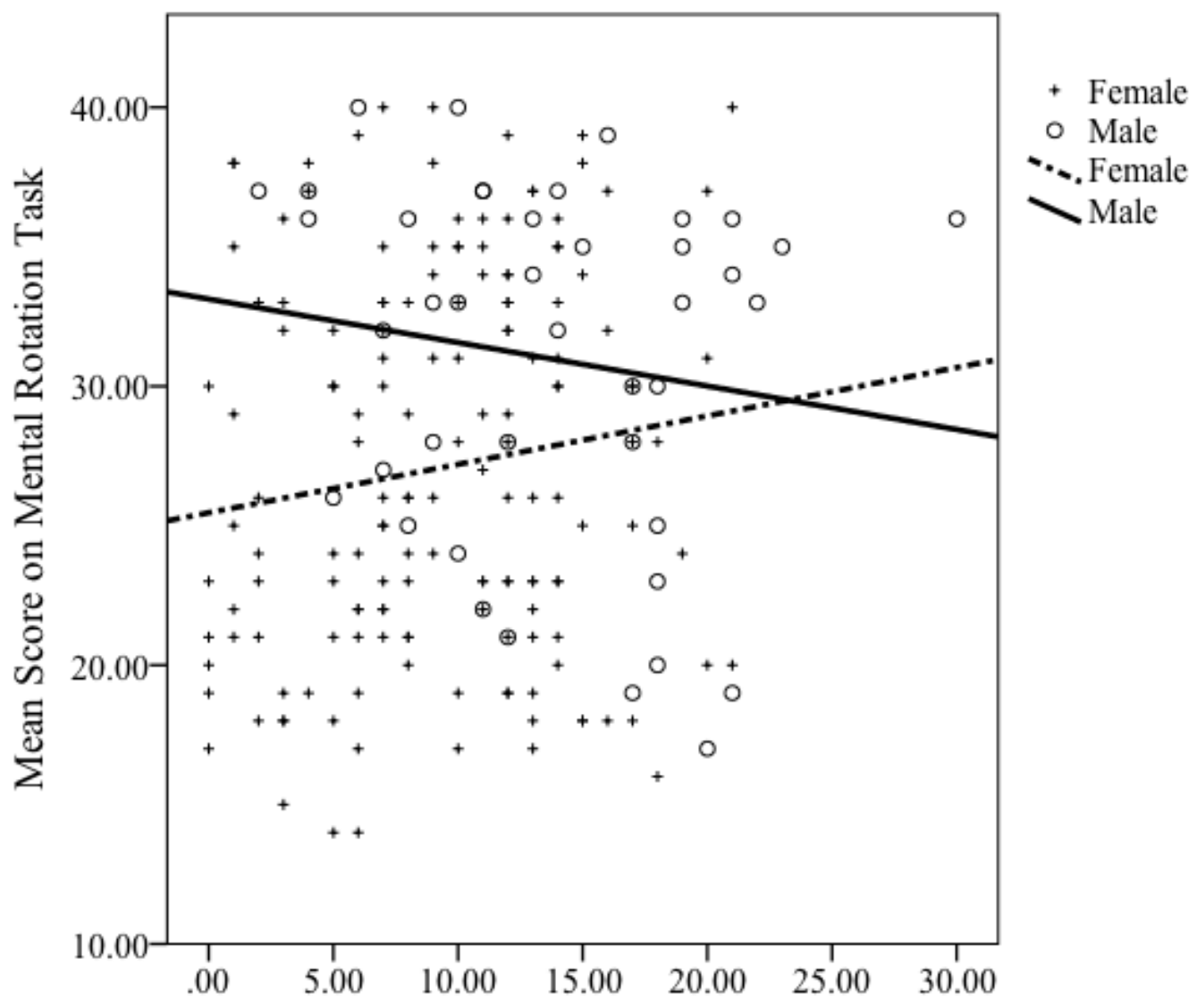

Number of Male Sex-typed Spatial Activities 
Figure 27. Mediation analysis investigating the role of early spatial activity experience in mental rotation performance. The numbers reported are $\beta$ values denoting standardized regression coefficients. $\left(n=67,{ }^{*} p \leq .05,{ }^{* *} p \leq .01,{ }^{* * *} p \leq .001\right)$.
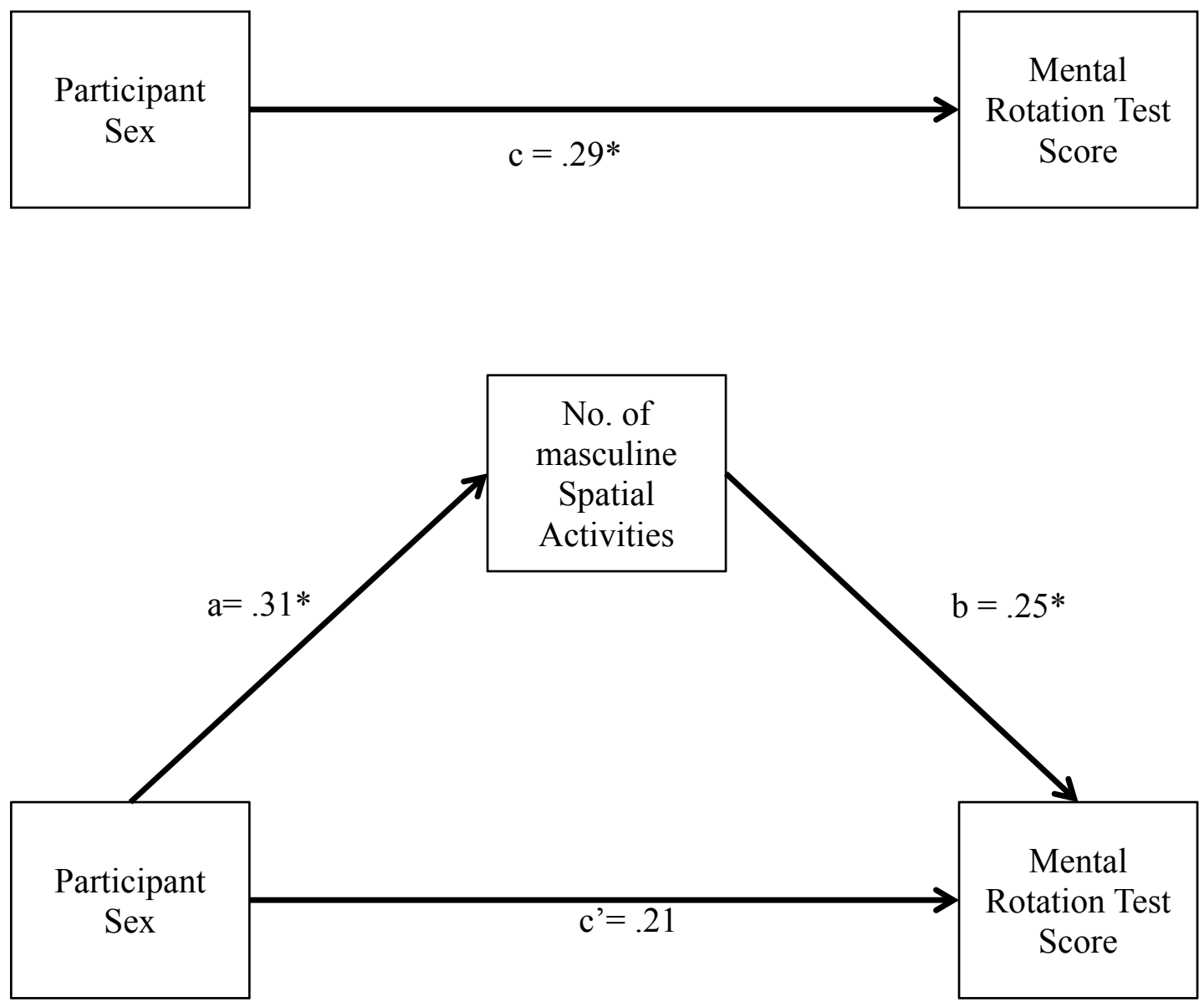
Figure 28. Relation between latent variable of cognitive strategy selection and early engagement in male sex-typed spatial activity experience.

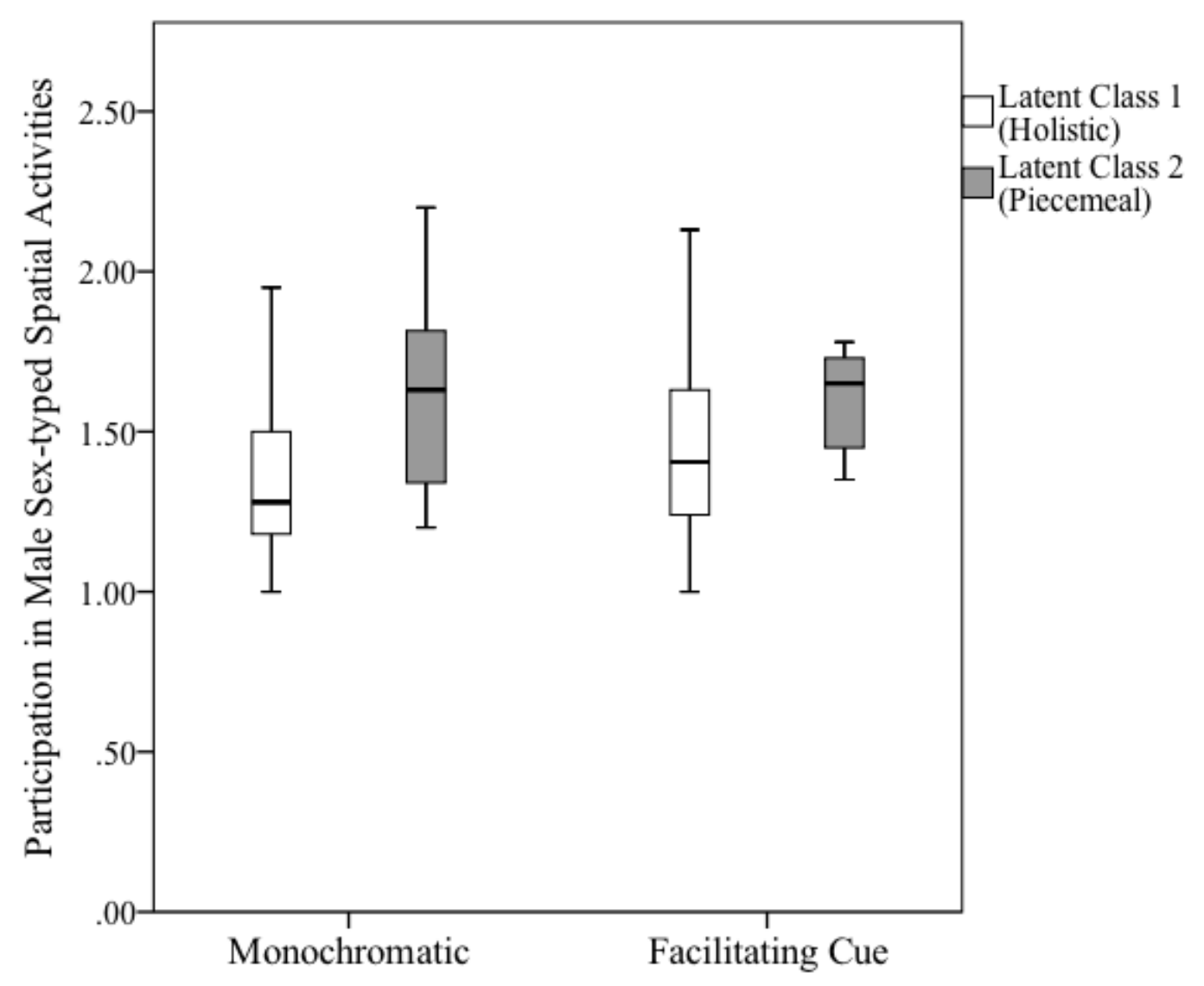


Figure 29. Sample plots of changepoints of two random participants to demonstrate PELT algorithm.
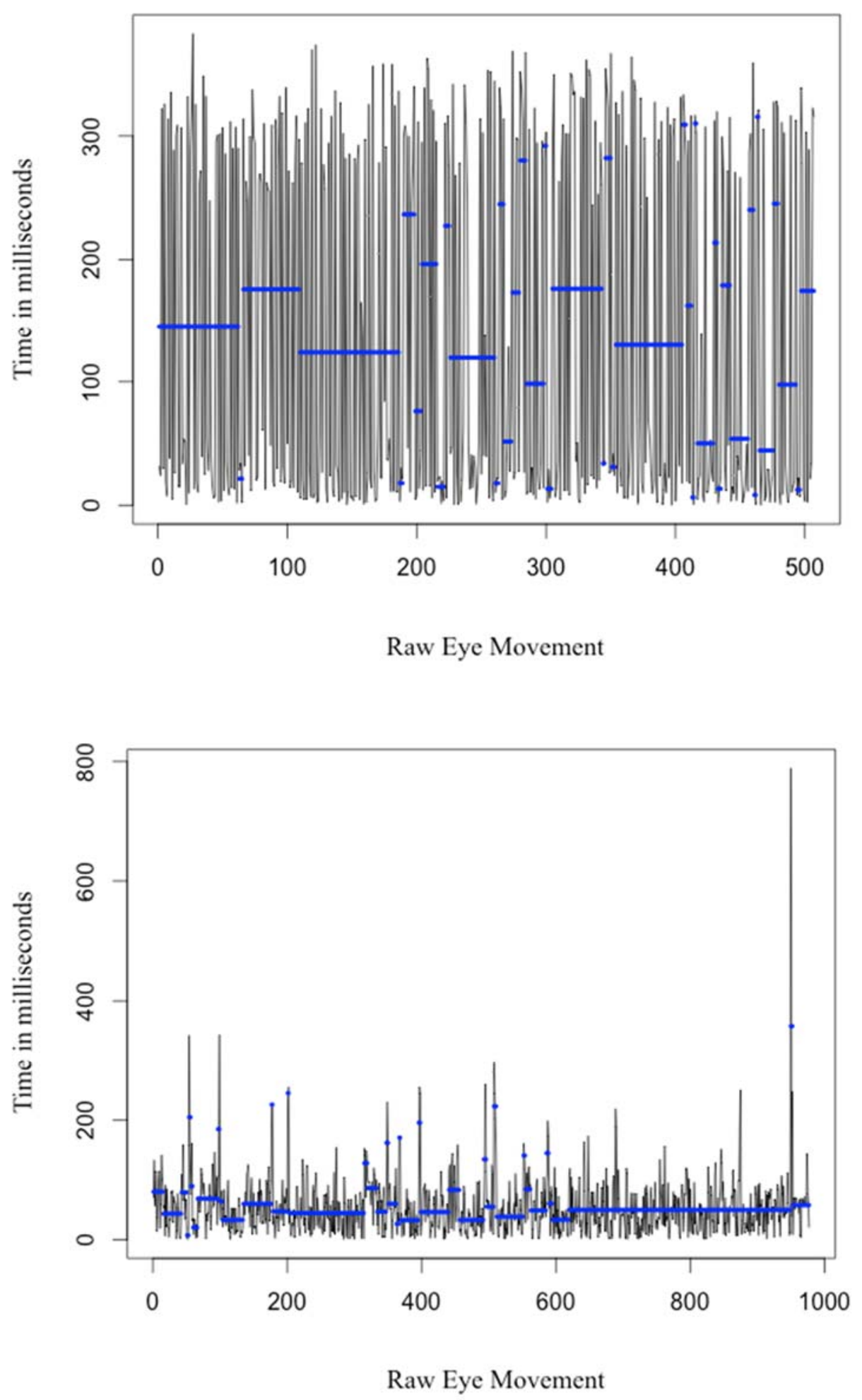
Figure 30. Relation between cognitive strategy flexibility (indicated by the number of changes detected in eye gaze patterns) and mental rotation performance in the monochromatic condition.

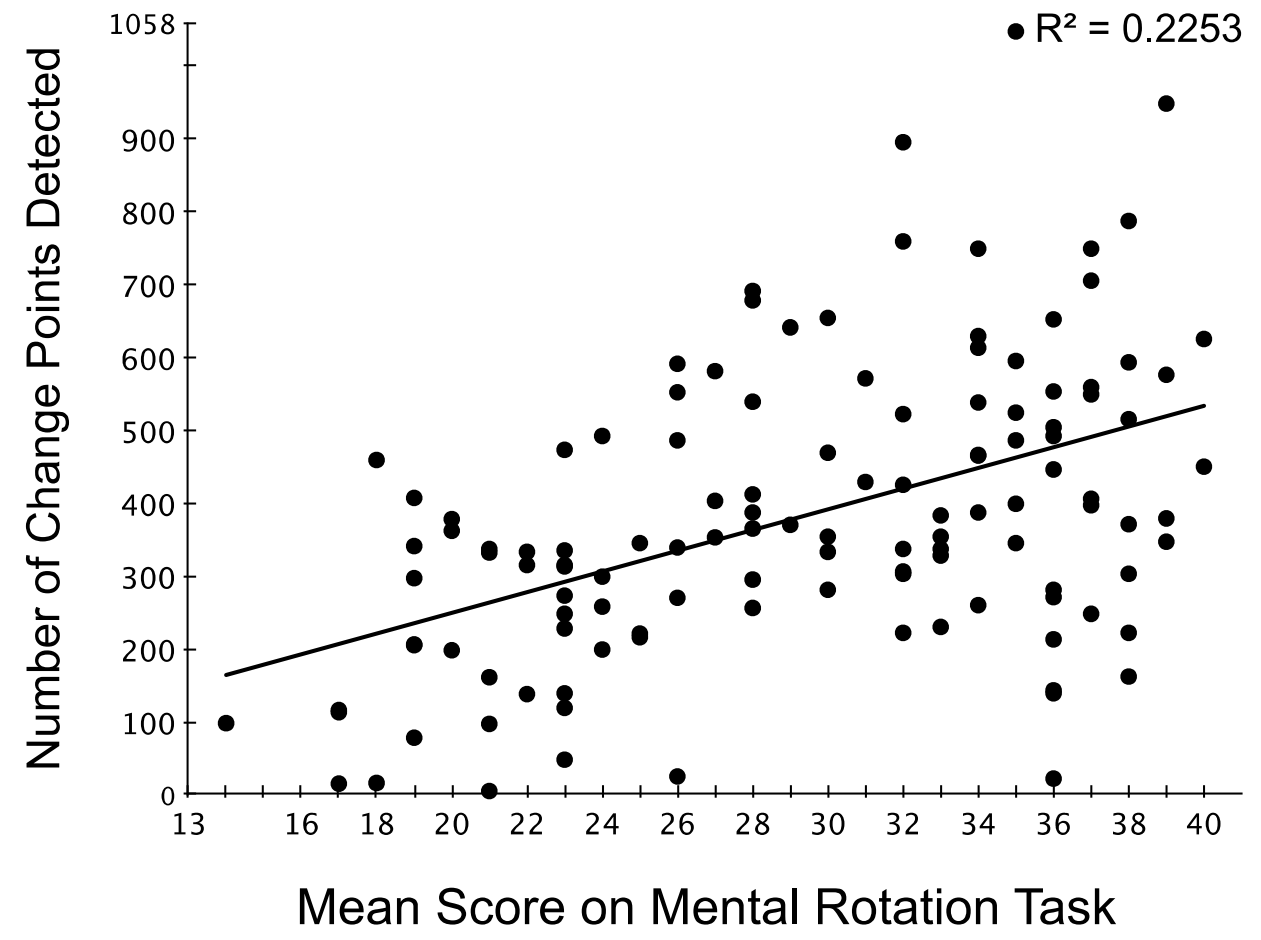


Figure 31. Relation between cognitive strategy flexibility (indicated by the number of changes detected in eye gaze patterns) and mental rotation performance in the facilitating cue (FC) condition.

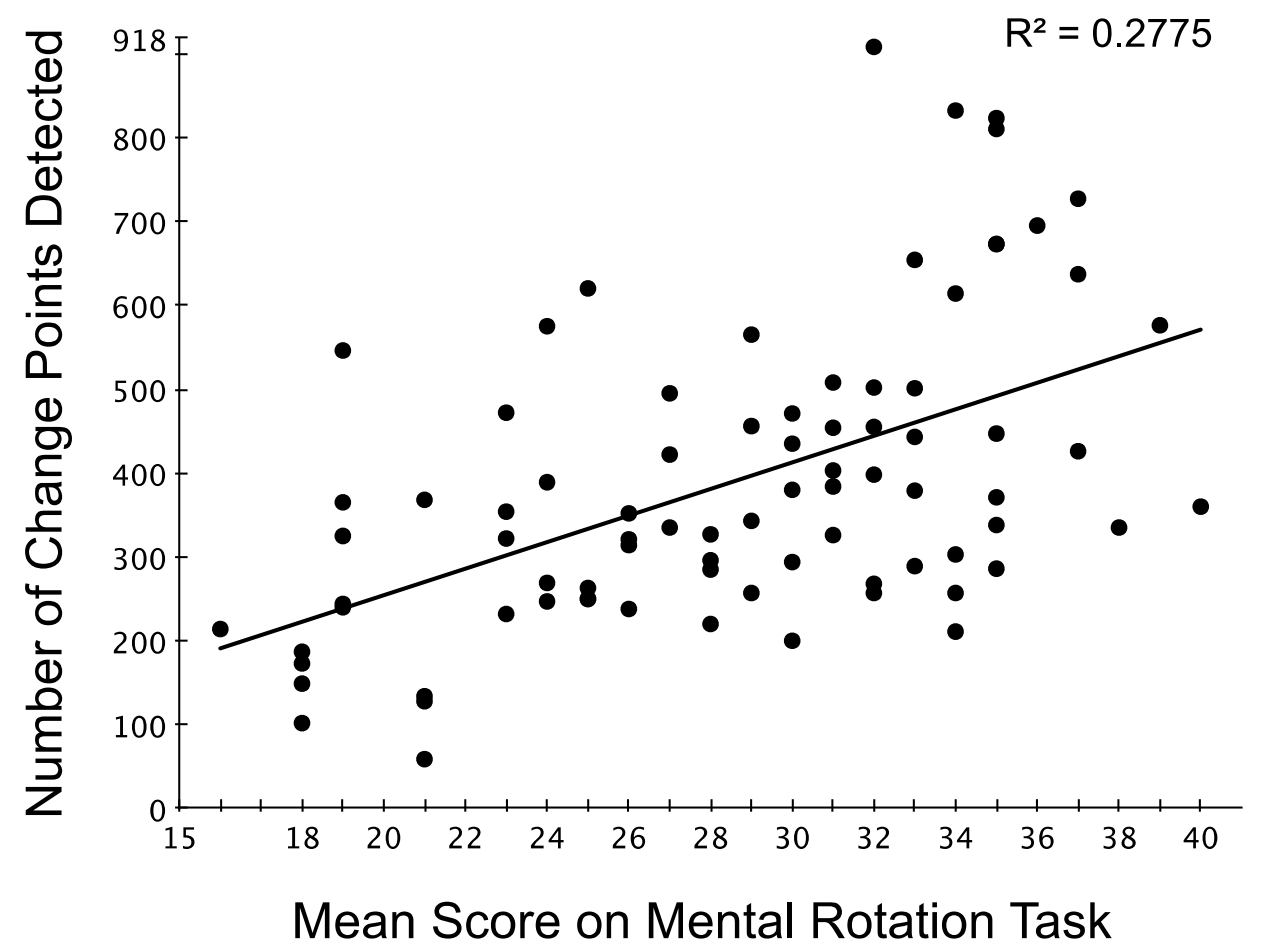


Figure 32. Relation between cognitive strategy flexibility (indicated by the number of changes detected in eye gaze patterns) and mental rotation performance in the inhibiting cue (IC) condition.

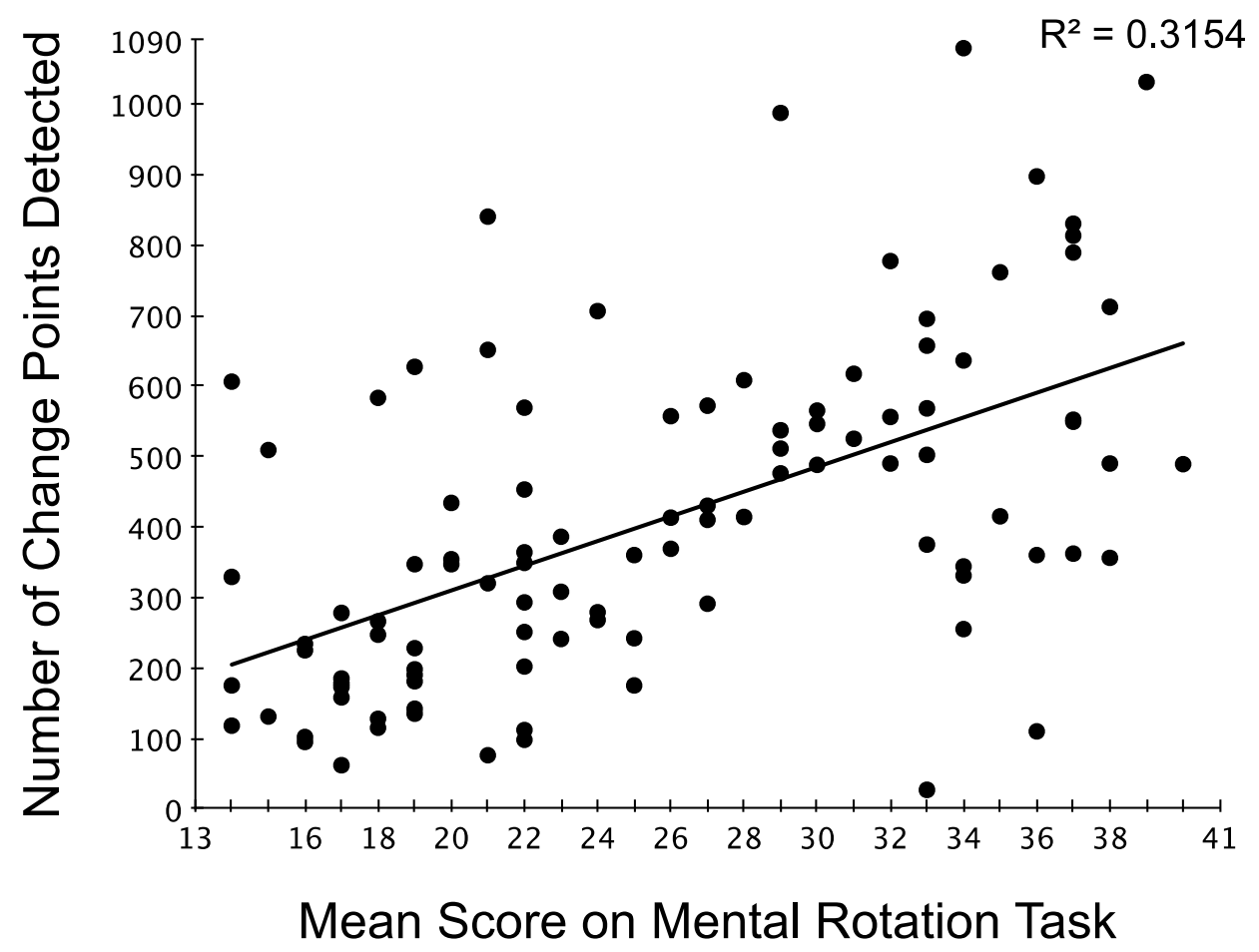


VITA

ALINA NAZARETH

2002-2006

Bachelor of Engineering in Electronics

Nagpur University, India

2006-2008

Post Graduate Diploma in Human Resource Management

T.A. Pai Management Institute, Manipal, India

2010-2012

M.S., Psychology

Florida International University, Miami, FL.

2012-2015

Doctoral Candidate, Psychology

Florida International University, Miami, FL.

\section{PUBLICATIONS AND PRESENTATIONS}

Vieites, V., Nazareth, A., Reeb-Sutherland, B.C., \& Pruden, S.M. (2015) A new biomarker to examine the role of hippocampal function in the development of spatial reorientation in children: A review. Frontiers in Psychology. 6:490 doi:

10.3389/fpsyg.2015.00490

Nazareth, A., Matos, N., Ramos, M., Waheed, I., \& Pruden, S.M. (2015, May) Early

Spatial Activity Experience Partially Mediates Adult Mental Rotation Performance. Poster presented at the 2015 South Florida Child Psychology Research Conference, Miami, FL.

Nazareth, A., \& Pruden, S.M. (2015, April). Beyond the playground: The Role of Spatial Activity Experience in Mental Rotation. Invited talk at Florida International University's Annual Scholarly Forum, Miami, FL.

Ramos, M., Matos, N., Waheed, I., Nazareth, A., \& Pruden, S.M. (2015, April). Susceptibility to Gender Stereotypes: An Indicator of Adult Mental Rotation Performance. Poster presented at the 2015 National Conference of Undergraduate Research (NCUR 2015) Cheney, WA.

Waheed, I., Nazareth, A., \& Pruden, S.M. (2015, March). Does Spatial Content Exposure affect Cognitive Strategy Selection? Invited talk at the FIU Florida Undergraduate Research Conference, Miami, FL.

Matos, N., Gallo, M., Nazareth, A., Ramos, M., \& Pruden, S.M. (2015, March). Memory for Spatial Locations: An Eye-tracking Study. Poster presented at the FIU Florida Undergraduate Research Conference, Miami, FL. 
Nazareth, A., Ramos, M., Labi, A.S., Humes, W.J., \& Pruden, S. M. (2014, November).

Individual Differences in Cognitive Strategy Selection during Mental Rotation: Role of Early Spatial Experience. Poster presented at the 2014 International Mind, Brain and Education Society, Fort Worth, TX.

Ramos, M., Nazareth, A., and Pruden S.M., (2014, October) Examining Stereotype Threat in Mental Rotation Performance. Invited talk at the McNair Scholars Research Conference, Miami, FL.

Nazareth, A., Pruden, S.M., \& Riedel, M. (2014, May). Raw Data Analyses Overcome Fixation Filter Constraints in Eye Tracking. Poster presented at the 26th Association for Psychological Science (APS) Annual Convention, San Francisco, CA.

Nazareth, A. (2014, May) Cognitive Strategy Selection during Mental Rotation based on Eye Movement Analyses. Invited talk at the First Annual Southeast CrossUniversity Mentoring Conference, Miami, FL.

Nazareth, A. (2014, April) Cognitive Factors Affecting Adult Mental Rotation Performance: An Eye-tracking Study. Invited talk at Florida International University's Annual Scholarly Forum, Miami, FL.

Nazareth, A., Herrera, A., \& Pruden, S.M. (2013). Explaining sex differences in mental rotation: Role of spatial activity experience. Cognitive Processing, doi: 10.1007/s10339013-0542-8

Nazareth, A., Bradley, C., Name, A., \& Pruden, S.M. (2013, November).Examining Sex Differences in Mental Rotation Strategy: An Eye-Tracking Study. Poster presented at the 8th Biennial meeting of the Society for the Study of Human Development (SSHD), Fort Lauderdale, FL.

Nazareth, A. (2013, September) Factors affecting mental rotation performance: An eyetracking study. Invited talk to the Center for Children and Families (CCF), Florida International University, Miami, FL.

Nazareth, A., Herrera, A., \& Pruden, S.M. (2013, April). Role of Spatial Activity Experience in Mental Rotation Ability. Poster presented at the 2013 Biennial Meeting for the Society for Research in Child Development (SRCD), Seattle, WA.

Nazareth, A. \& Pruden, S.M. (2013, March) Sex Differences in Mental Rotation: Are women playing the right games? Invited talk to the 2013 Women, Sexuality and Gender Student Association (WGSA) Annual Student Conference, Miami, FL. 